\title{
Total Synthesis of (-)-Hennoxazole
}

Thomas E. Smith,* Wen-Hsin Kuo, Emily P. Balskus, Victoria D. Bock, Jennifer L. Roizen, Ashleigh B. Theberge, Kathleen A. Carroll, Tomoki Kurihara, and Jeffrey D. Wessler

Department of Chemistry, Williams College, Williamstown, Massachusetts 01267

Supporting Information

\section{Table of Contents}

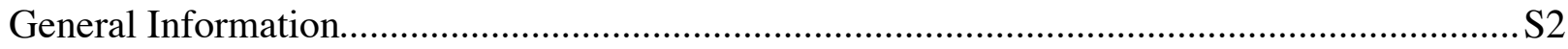

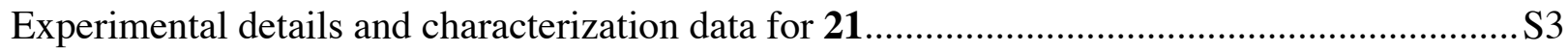

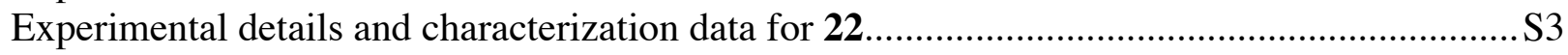

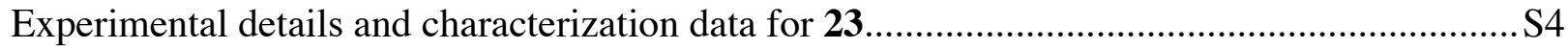

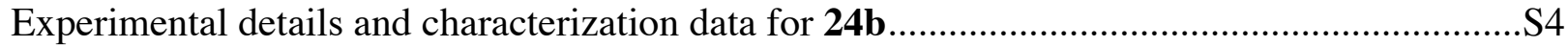

Experimental details and characterization data for 40...................................................... S5

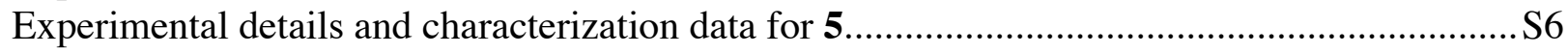

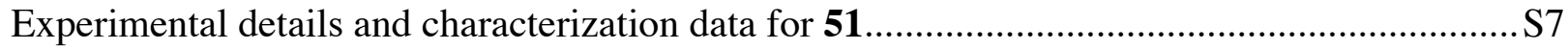

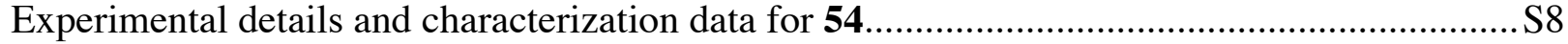

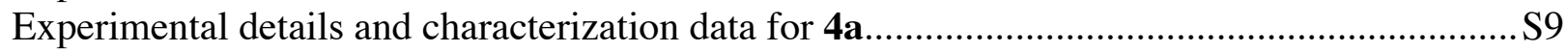

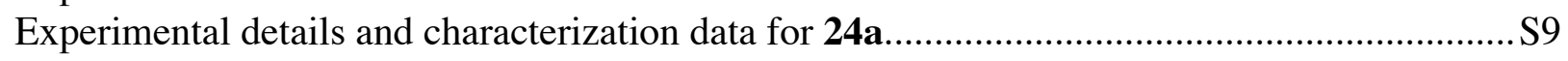

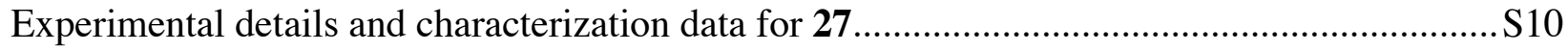

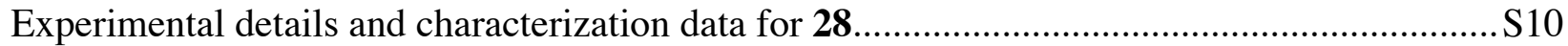

Experimental details and characterization data for 29......................................................S11

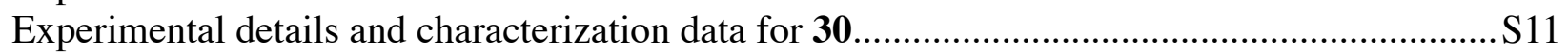

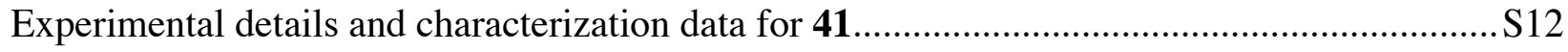

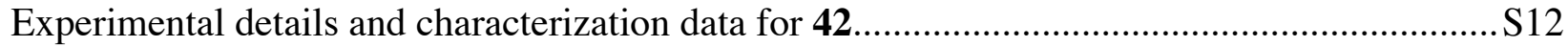

General procedure for $\mathrm{TiCl}_{4} /(i \text {-Pr })_{2} \mathrm{NEt}$ aldol-type reactions with acetals.............................S13

General procedure for $\mathrm{PhBCl}_{2} /$ sparteine aldol-type reactions with acetals.............................S13

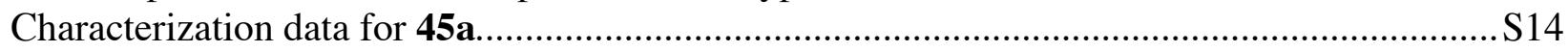

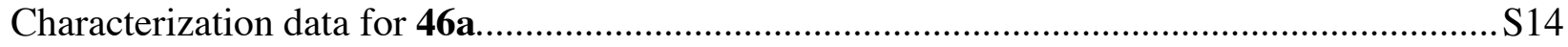

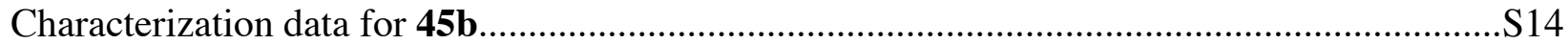

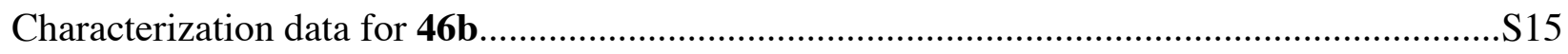

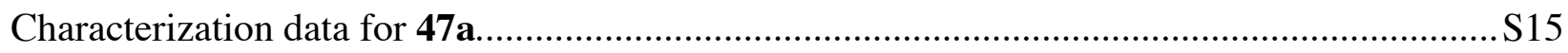

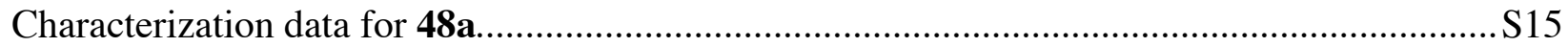

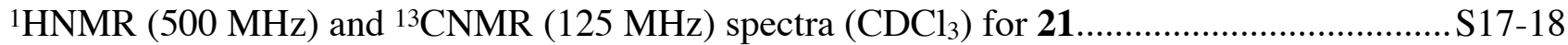

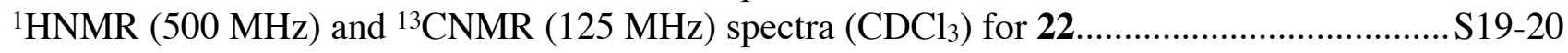

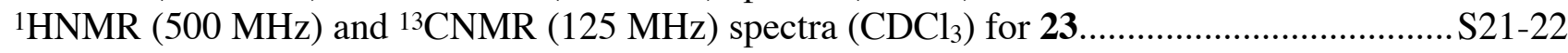

${ }^{1} \mathrm{HNMR}(500 \mathrm{MHz})$ and ${ }^{13} \mathrm{CNMR}(125 \mathrm{MHz})$ spectra $\left(\mathrm{CDCl}_{3}\right)$ for $\mathbf{2 4 b}$...............................S23-24

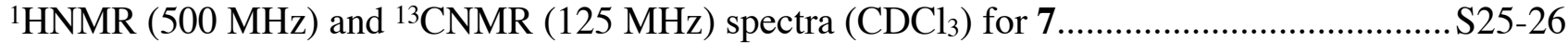

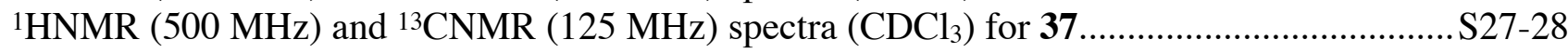

${ }^{1} \mathrm{HNMR}(500 \mathrm{MHz})$ and ${ }^{13} \mathrm{CNMR}(125 \mathrm{MHz})$ spectra $\left(\mathrm{CDCl}_{3}\right)$ for 40................................ S29-30

${ }^{1} \mathrm{HNMR}(500 \mathrm{MHz})$ and ${ }^{13} \mathrm{CNMR}(125 \mathrm{MHz})$ spectra $\left(\mathrm{CDCl}_{3}\right)$ for 5.................................. S31-32

${ }^{1} \mathrm{HNMR}(500 \mathrm{MHz})$ and ${ }^{13} \mathrm{CNMR}(125 \mathrm{MHz})$ spectra $\left(\mathrm{CDCl}_{3}\right)$ for $47 \mathbf{b} \ldots \ldots \ldots \ldots \ldots \ldots \ldots \ldots \ldots \ldots . . . . . . . . . . . . . . . . .333-34$

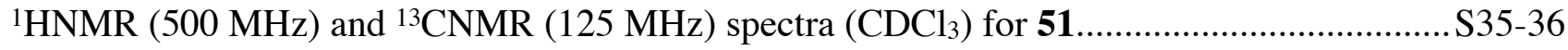

${ }^{1} \mathrm{HNMR}(500 \mathrm{MHz})$ and ${ }^{13} \mathrm{CNMR}(125 \mathrm{MHz})$ spectra $\left(\mathrm{CDCl}_{3}\right)$ for 53............................... $337-38$ 
${ }^{1} \mathrm{HNMR}(500 \mathrm{MHz})$ and ${ }^{13} \mathrm{CNMR}(125 \mathrm{MHz})$ spectra $\left(\mathrm{CDCl}_{3}\right)$ for $\mathbf{5 4}$ S39-40

${ }^{1} \mathrm{HNMR}(500 \mathrm{MHz})$ and ${ }^{13} \mathrm{CNMR}(125 \mathrm{MHz})$ spectra $\left(\mathrm{CDCl}_{3}\right)$ for $\mathbf{4 a}$ S41-42

${ }^{1} \mathrm{HNMR}(500 \mathrm{MHz})$ and ${ }^{13} \mathrm{CNMR}(125 \mathrm{MHz})$ spectra $\left(\mathrm{CDCl}_{3}\right)$ for $\mathbf{5 5}$ S43-44

${ }^{1} \mathrm{HNMR}(500 \mathrm{MHz})$ and ${ }^{13} \mathrm{CNMR}(125 \mathrm{MHz})$ spectra $\left(\mathrm{CDCl}_{3}\right)$ for $\mathbf{1 a}$ $\mathrm{S} 45-46$

${ }^{1} \mathrm{HNMR}(500 \mathrm{MHz})$ and ${ }^{13} \mathrm{CNMR}(125 \mathrm{MHz})$ spectra (acetone- $\left.d_{6}\right)$ for $\mathbf{1 a}$ S47-48

${ }^{1} \mathrm{HNMR}(500 \mathrm{MHz})$ and ${ }^{13} \mathrm{CNMR}(125 \mathrm{MHz})$ spectra $\left(\mathrm{CDCl}_{3}\right)$ for $\mathbf{2 4 a}$ S49-50 ${ }^{1} \mathrm{HNMR}(500 \mathrm{MHz})$ and ${ }^{13} \mathrm{CNMR}(125 \mathrm{MHz})$ spectra $\left(\mathrm{CDCl}_{3}\right)$ for 27 S51-52

${ }^{1} \mathrm{HNMR}(500 \mathrm{MHz})$ and ${ }^{13} \mathrm{CNMR}(125 \mathrm{MHz})$ spectra $\left(\mathrm{CDCl}_{3}\right)$ for 28 S53-54

${ }^{1} \mathrm{HNMR}(500 \mathrm{MHz})$ and ${ }^{13} \mathrm{CNMR}(125 \mathrm{MHz})$ spectra $\left(\mathrm{CDCl}_{3}\right)$ for 29 S55-56

${ }^{1} \mathrm{HNMR}(500 \mathrm{MHz})$ and ${ }^{13} \mathrm{CNMR}(125 \mathrm{MHz})$ spectra $\left(\mathrm{CDCl}_{3}\right)$ for $\mathbf{3 0}$ S57-58 ${ }^{1} \mathrm{HNMR}(500 \mathrm{MHz})$ and ${ }^{13} \mathrm{CNMR}(125 \mathrm{MHz})$ spectra $\left(\mathrm{CDCl}_{3}\right)$ for 41 S59-60

${ }^{1} \mathrm{HNMR}(500 \mathrm{MHz})$ and ${ }^{13} \mathrm{CNMR}(125 \mathrm{MHz})$ spectra $\left(\mathrm{CDCl}_{3}\right)$ for 42 S61-62

${ }^{1} \mathrm{HNMR}(500 \mathrm{MHz})$ and ${ }^{13} \mathrm{CNMR}(125 \mathrm{MHz})$ spectra $\left(\mathrm{CDCl}_{3}\right)$ for $\mathbf{4 5 a}$ S63-64

${ }^{1} \mathrm{HNMR}(500 \mathrm{MHz})$ and ${ }^{13} \mathrm{CNMR}(125 \mathrm{MHz})$ spectra $\left(\mathrm{CDCl}_{3}\right)$ for $\mathbf{4 6 a}$ S65-66 ${ }^{1} \mathrm{HNMR}(500 \mathrm{MHz})$ and ${ }^{13} \mathrm{CNMR}(125 \mathrm{MHz})$ spectra $\left(\mathrm{CDCl}_{3}\right)$ for $\mathbf{4 5 b}$ S67-68 ${ }^{1} \mathrm{HNMR}(500 \mathrm{MHz})$ and ${ }^{13} \mathrm{CNMR}(125 \mathrm{MHz})$ spectra $\left(\mathrm{CDCl}_{3}\right)$ for $\mathbf{4 6} \mathrm{b}$ S69-70 ${ }^{1} \mathrm{HNMR}(500 \mathrm{MHz})$ and ${ }^{13} \mathrm{CNMR}(125 \mathrm{MHz})$ spectra $\left(\mathrm{CDCl}_{3}\right)$ for $47 \mathbf{a}$ $\mathrm{S} 71-72$ ${ }^{1} \mathrm{HNMR}(500 \mathrm{MHz})$ and ${ }^{13} \mathrm{CNMR}(125 \mathrm{MHz})$ spectra $\left(\mathrm{CDCl}_{3}\right)$ for $48 \mathrm{a}$ S73-74

General Information. Oxygen- or moisture-sensitive reactions were carried out in flame-dried or ovendried glassware sealed with rubber septa under a positive pressure of dry nitrogen. Similarly sensitive liquids and solutions were transferred by gas-tight syringe or cannula. Unless indicated otherwise, reagents and solvents were purchased and used without purification. Ether, THF, and $\mathrm{CH}_{2} \mathrm{Cl}_{2}, \mathrm{were}$ purified by passage through a bed of activated alumina. ${ }^{1}$ Analytical TLC was performed with $0.25 \mathrm{~mm}$ silica gel 60 plates with $254 \mathrm{~nm}$ fluorescent indicator from SiliCycle. Plates were visualized under UV light and treatment with either acidic $p$-anisaldehyde stain or aqueous ceric ammonium molybdate (CAM) solution followed by gentle heating. The term flash chromatography refers to preparative silica gel column chromatography as described by Still and co-workers. ${ }^{2}$ Silica gel 60, 230-240 mesh, was purchased from SiliCycle (R10030B). ${ }^{1} \mathrm{H}$ NMR spectra are reported in ppm using tetramethylsilane (0.00 ppm) or solvent $\left(\mathrm{CDCl}_{3}: 7.24 \mathrm{ppm}\right.$; acetone- $\left.\mathrm{d}_{6}: 2.04 \mathrm{ppm}\right)$ as an internal standard. Data are reported as $(\mathrm{ap}=$ apparent, $\mathrm{s}=$ singlet, $\mathrm{d}=$ doublet, $\mathrm{t}=$ triplet, $\mathrm{q}=$ quartet, $\mathrm{m}=$ multiplet, $\mathrm{b}=$ broad; coupling constant(s) in $\mathrm{Hz}$; integration. Proton-decoupled ${ }^{13} \mathrm{C}$ NMR spectra were recorded at $125 \mathrm{MHz}$ and are reported in ppm using solvent as an internal standard $\left(\mathrm{CDCl}_{3}: 77.00 \mathrm{ppm}\right.$, acetone- $\mathrm{d}_{6}: 206.00$ $\mathrm{ppm})$. Unless noted otherwise on the spectra, NMR spectra are recorded in $\mathrm{CDCl}_{3}$. Infrared spectra were recorded as thin films on $\mathrm{NaCl}$ plates on a Fourier transform spectrometer (FTIR). Melting points are uncorrected. Optical rotations were measured using a sodium (589, D line) lamp and are reported as follows: $[\alpha]_{\lambda}{ }^{\circ} \mathrm{C}(\mathrm{c}=\mathrm{g} / 100 \mathrm{~mL}$, solvent $)$.

(1) Pangborn, A. B.; Giardello, M. A.; Grubbs, R. H.; Rosen, R. K.; Timmers, F. J. Organometallics 1996, 1518.

(2) Still, W. C.; Kahn, M.; Mitra, A. J. Org. Chem 1978, 43, 2923. 


\section{Bisoxazole Methyl Ester 21. ${ }^{3}$}
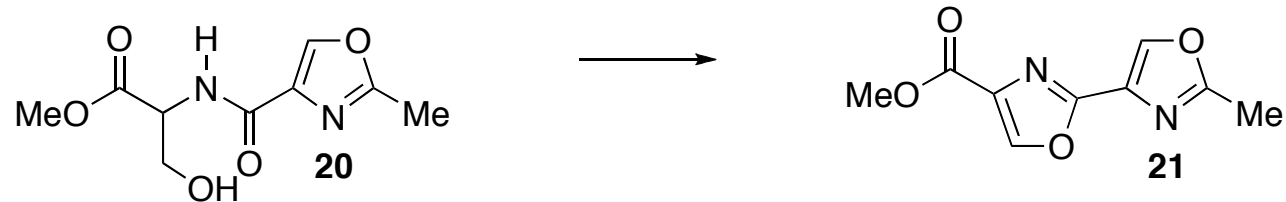

Following Wipf \& Williams' method, ${ }^{4}$ a solution of serine amide 20 (1.000 g, $4.38 \mathrm{mmol}, 1$ equiv) in $\mathrm{CH}_{2} \mathrm{Cl}_{2}(40 \mathrm{~mL})$ was cooled to $-20{ }^{\circ} \mathrm{C}$ and Deoxo-Fluor $(0.89 \mathrm{~mL}, 4.82 \mathrm{mmol}, 1.1 \mathrm{equiv})$ was added dropwise. After $15 \mathrm{~min}$, TLC showed complete consumption of starting material. Subsequent addition of $\mathrm{BrCCl}_{3}$ (1.56 mL, $15.8 \mathrm{mmol}, 3.6$ equiv) followed by DBU (2.35 mL, $15.8 \mathrm{mmol}, 3.6$ equiv) led to an immediate darkening of color. The reaction mixture was allowed to warm to $0{ }^{\circ} \mathrm{C}$ and was stirred at that temp for $3 \mathrm{~h}$. The reaction was quenched by the addition of sat $\mathrm{NaHCO}_{3}(40 \mathrm{~mL})$ at $0{ }^{\circ} \mathrm{C}$, and the resulting suspension was extracted with EtOAc $(3 \times 50 \mathrm{~mL})$. The combined organics were dried over $\mathrm{MgSO}_{4}$, filtered, and the solvent removed in vacuo to provide the crude product as a light brown solid. Silica gel chromatography (60\% EtOAc/hexanes; TLC: $\mathrm{R}_{\mathrm{f}}=0.57$ in $100 \%$ EtOAc, CAM stain) gave bisoxazole methyl ester 21 (752 mg, 82\%) as a white solid. ${ }^{1} \mathrm{H}$ NMR (500 MHz, CDCl $\left.{ }_{3}\right) \delta 8.30(\mathrm{~s}, 1 \mathrm{H})$, 8.28 (s, 1H), 3.95 (s, 3H), 2.56 (s, 3H) ppm; $\left.{ }^{13} \mathrm{C} \mathrm{NMR} \mathrm{(125} \mathrm{MHz,} \mathrm{CDCl}_{3}\right) \delta 162.8,161.3,155.8,143.5$, 139.2, 134.2, 129.6, 52.2, $13.7 \mathrm{ppm}$.

\section{Preparation of Bisoxazole Aldehyde 22.5}
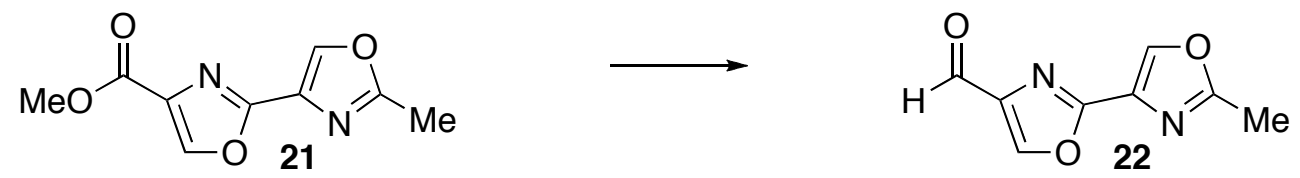

To a solution of bisoxazole ester 21 (328 mg, $1.58 \mathrm{mmol}, 1$ equiv) in $\mathrm{CH}_{2} \mathrm{Cl}_{2}(50 \mathrm{~mL})$ at $-78{ }^{\circ} \mathrm{C}$ was added DIBAL-H (3.94 mL of a $1.0 \mathrm{M}$ solution in $\left.\mathrm{CH}_{2} \mathrm{Cl}_{2}, 3.94 \mathrm{mmol}, 2.5 \mathrm{mmol}\right)$ dropwise. The reaction mixture was stirred at $-78{ }^{\circ} \mathrm{C}$ for $20 \mathrm{~min}$ and was then quenched by the addition of methanol $(10 \mathrm{~mL})$ and half-saturated aq Rochelle's salt. The resulting suspension was allowed to warm to rt and was stirred for $5 \mathrm{~h}$ until two distinct layers formed. The layers were separated and the aqueous layer was extracted with EtOAc $(2 \times 40 \mathrm{~mL})$. The combined organics were dried over $\mathrm{MgSO}_{4}$, filtered, and the solvent removed in vacuo to provide bisoxazole aldehyde $22(287 \mathrm{mg}, 100 \%)$ as a yellow solid, which was used without further purification (TLC: $\mathrm{R}_{\mathrm{f}}=0.42$ in $40 \% \mathrm{Et}_{2} \mathrm{O} / \mathrm{CH}_{2} \mathrm{Cl}_{2}, \mathrm{UV}, \mathrm{CAM}$ stain). $\mathrm{Mp} 175.5-175.8{ }^{\circ} \mathrm{C}$; ${ }^{1} \mathrm{H}$ NMR $\left(500 \mathrm{MHz}, \mathrm{CDCl}_{3}\right) \delta 10.00(\mathrm{~s}, 1 \mathrm{H}), 8.32(\mathrm{~s}, 1 \mathrm{H}), 8.24(\mathrm{~s}, 1 \mathrm{H}), 2.57(\mathrm{~s}, 3 \mathrm{H}) \mathrm{ppm} ;{ }^{13} \mathrm{C} \mathrm{NMR}$ $\left(125 \mathrm{MHz}, \mathrm{CDCl}_{3}\right) \delta 184.1,163.1,156.3,143.5,141.5,139.3,129.5,13.7$ ppm; IR (film) 3124, 1686, 1295, $1206 \mathrm{~cm}^{-1}$; HRMS (EI): Exact mass calcd for $\mathrm{C}_{8} \mathrm{H}_{6} \mathrm{~N}_{2} \mathrm{O}_{3}[\mathrm{M}]^{+}$: 178.0378; Found: 178.0387. Elemental analysis calcd for $\mathrm{C}_{8} \mathrm{H}_{6} \mathrm{~N}_{2} \mathrm{O}_{3}$ : C, 53.94; H, 3.39; N, 15.73; Found: C, 54.08; H, 3.45; N, 15.70.

(3) Chattopadhyay, S. K.; Kempson, J.; McNeil, A.; Pattenden, G.; Reader, M.; Rippon, D. E.; Waite, D. J. Chem. Soc., Perkin Trans. 1, 2000, 2415-2428.

(4) Phillips, A. J.; Uto, Y.; Wipf, P.; Reno, M. J.; Williams, D. R. Org. Lett. 2000, 2, 1165-1168.

(5) Williams, D. R.; Brooks, D. A.; Moore, J. L.; A. Stewart, A. O. Tetrahedron Lett. 1996, 37, 983-986. 


\section{Bisoxazole Dimethylacetal 23.}
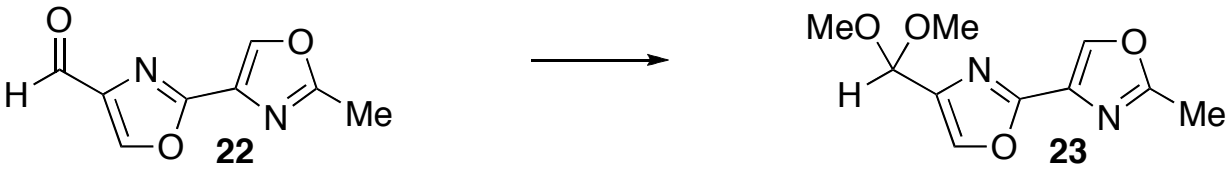

To a solution of bisoxazole aldehyde $22\left(502 \mathrm{mg}, 2.82 \mathrm{mmol}, 1\right.$ equiv) in $\mathrm{CHCl}_{3}(20 \mathrm{~mL}$, it is essential to select a grade that is not stabilized with ethanol) at $-30{ }^{\circ} \mathrm{C}$ was added TMSOMe $(1.16 \mathrm{~mL}, 8.45$ mmol, 3.0 equiv) followed by TMSOTf ( $153 \mu \mathrm{L}, 0.845 \mathrm{mmol}, 0.3$ equiv) and the mixture turned slightly cloudy and took on a deeper orange color. After $14 \mathrm{~h}$ at $-30{ }^{\circ} \mathrm{C}$, an additional 1.0 equiv of TMSOMe and 0.1 equiv TMSOTf were added and the reaction was stirred for another $24 \mathrm{~h}$ before quenching with pyridine $(1 \mathrm{~mL})$. The mixture was warmed to $\mathrm{rt}$ and was poured into sat aq $\mathrm{NaHCO}_{3}(40 \mathrm{~mL})$ and was extracted with $\mathrm{CH}_{2} \mathrm{Cl}_{2}(2 \times 40 \mathrm{~mL})$. The combined organic layers were dried over a 1:1 mixture of $\mathrm{K}_{2} \mathrm{CO}_{3}$ and $\mathrm{Na}_{2} \mathrm{SO}_{4}$, filtered, and concentrated in vacuo to yield a white solid. Purification by silica gel chromatography ( $\mathrm{Et}_{3} \mathrm{~N}$-deactivated silica gel, 1:1:1 Et ${ }_{2} \mathrm{O} / \mathrm{CH}_{2} \mathrm{Cl}_{2} /$ hexanes; TLC: $\mathrm{R}_{\mathrm{f}}=0.71,100 \% \mathrm{Et}_{2} \mathrm{O}$, UV) provided bisoxazole dimethylacetal $23(609 \mathrm{mg}, 96 \%)$ as a white solid. Mp 63.5-64.0 ${ }^{\circ} \mathrm{C}$; ${ }^{1} \mathrm{H}$ NMR $\left(500 \mathrm{MHz}, \mathrm{CDCl}_{3}\right) \delta 8.17(\mathrm{~s}, 1 \mathrm{H}), 7.72(\mathrm{~s}, 1 \mathrm{H}), 5.48(\mathrm{~s}, 1 \mathrm{H}), 3.39(\mathrm{~s}, 6 \mathrm{H}), 2.54(\mathrm{~s}, 3 \mathrm{H}) \mathrm{ppm} ;{ }^{13} \mathrm{C} \mathrm{NMR}$ $\left(125 \mathrm{MHz}, \mathrm{CDCl}_{3}\right) \delta 162.6,155.5,139.5,138.2,136.8,130.3,98.3,52.8,13.7$ ppm; IR (film) 3119, 1636, 1530, 1305, 1106, 1058, $984 \mathrm{~cm}^{-1}$; HRMS (EI): Exact mass calcd for $\mathrm{C}_{10} \mathrm{H}_{12} \mathrm{~N}_{2} \mathrm{O}_{4}[\mathrm{M}]^{+}:$: 224.0797; Found: 224.0794. Elemental analysis calcd for $\mathrm{C}_{10} \mathrm{H}_{12} \mathrm{~N}_{2} \mathrm{O}_{4}$ : C, 53.57; H, 5.39; N, 12.49; Found: C, $53.68 ; \mathrm{H}, 5.41 ; \mathrm{N}, 12.41$.

\section{TBS-Protected Bisoxazole Dimethylacetal 24b.}
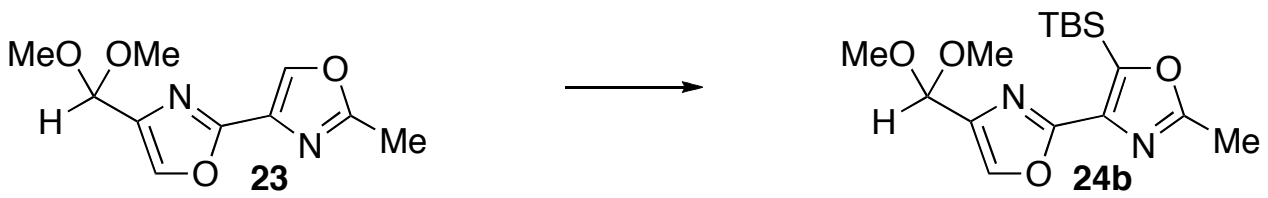

To bisoxazole dimethylacetal 23 (649 $\mathrm{mg}, 2.89 \mathrm{mmol}, 1$ equiv) in dry THF $(29 \mathrm{~mL})$ at $-78{ }^{\circ} \mathrm{C}$ was added $n$-BuLi (2.5 M in hexanes, $1.27 \mathrm{~mL}, 3.18 \mathrm{mmol}, 1.1$ equiv). The reaction mixture gradually took on a bright yellow color. After stirring at $-78^{\circ} \mathrm{C}$ for $30 \mathrm{~min}$, TBSOTf (0.696 mL, $3.03 \mathrm{mmol}, 1.05$ equiv) was added dropwise. The reaction mixture was stirred at $-78{ }^{\circ} \mathrm{C}$ and the color slowly faded from yellow to almost colorless. After $40 \mathrm{~min}$, TLC indicated some unreacted SM. An additional amount of TBSOTf ( $0.100 \mathrm{~mL}, 0.435 \mathrm{mmol}, 0.15$ equiv) was added. After 20 minutes, TLC showed complete consumption of SM. The reaction was quenched by the addition of sat aq $\mathrm{NaHCO}_{3}(30 \mathrm{~mL})$ and the resulting suspension was warmed to rt and extracted with $\mathrm{CH}_{2} \mathrm{Cl}_{2}(3 \times 30 \mathrm{~mL})$. The combined organic layers were dried over a 1:1 mixture of $\mathrm{K}_{2} \mathrm{CO}_{3}$ and $\mathrm{Na}_{2} \mathrm{SO}_{4}$, filtered, and concentrated in vacuo to yield a colorless solid. Purification by silica gel chromatography $\left(15 \rightarrow 55 \% \mathrm{Et}_{2} \mathrm{O} /\right.$ hexanes; TLC: $\mathrm{R}_{\mathrm{f}}=0.74,80 \%$ ether/ hexanes, UV) provided TBS-protected bisoxazole dimethylacetal $\mathbf{2 4 b}(860 \mathrm{mg}, 88 \%)$ as a colorless, crystalline solid. Mp 89.2-90.8 ${ }^{\circ} \mathrm{C} ;{ }^{1} \mathrm{H}$ NMR $\left(500 \mathrm{MHz}, \mathrm{CDCl}_{3}\right) \delta 7.70(\mathrm{~s}, 1 \mathrm{H}), 5.47(\mathrm{~s}, 1 \mathrm{H}), 3.38(\mathrm{~s}$, $6 \mathrm{H}), 2.54(\mathrm{~s}, 3 \mathrm{H}), 0.95(\mathrm{~s}, 9 \mathrm{H}), 0.38(\mathrm{~s}, 6 \mathrm{H}) \mathrm{ppm} ;{ }^{13} \mathrm{C} \mathrm{NMR}\left(125 \mathrm{MHz}, \mathrm{CDCl}_{3}\right) \delta 165.1,156.6,154.8$, 139.6, 136.5, 98.6, 52.7, 26.4, 17.6, 13.7, -5.9 ppm; IR (film) 2929, 1611, 1114, 1101, $1061 \mathrm{~cm}^{-1}$; HRMS (EI): Exact mass calcd for $\mathrm{C}_{15} \mathrm{H}_{23} \mathrm{~N}_{2} \mathrm{O}_{3} \mathrm{Si}\left[\mathrm{M}-\mathrm{OCH}_{3}\right]^{+}$: 307.1478; Found: 307.1492. Elemental analysis calcd for $\mathrm{C}_{16} \mathrm{H}_{26} \mathrm{~N}_{2} \mathrm{O}_{4} \mathrm{Si}$ : C, 56.78; H, 7.74; N, 8.28; Found: C, 56.98; H, 7.67; N, 8.29. 


\section{Trienyl Alcohol 40.}

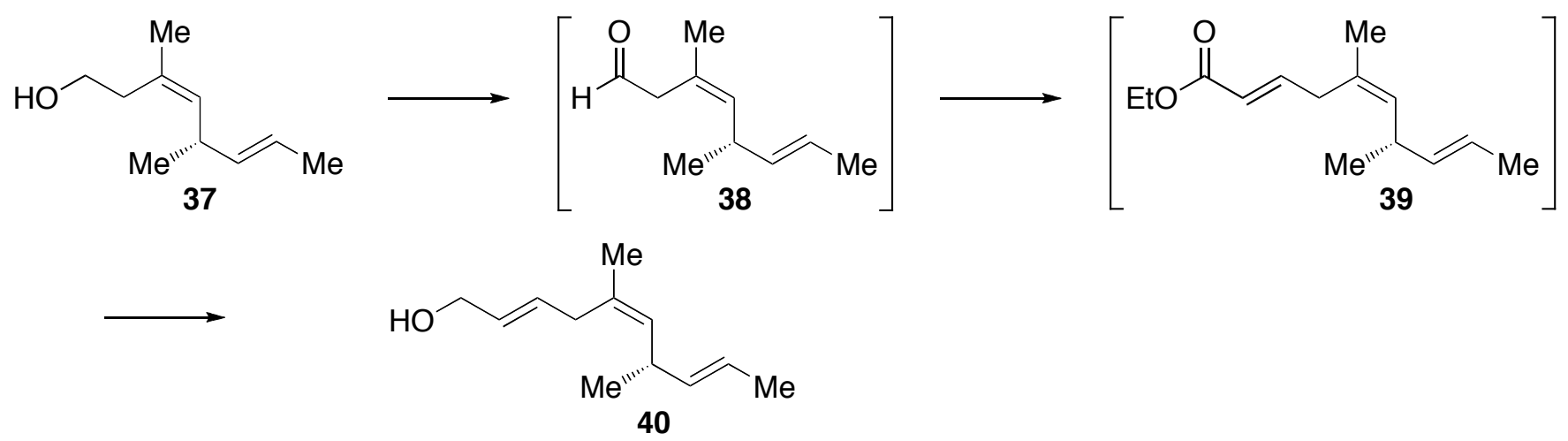

(a) Oxidation. A $25 \mathrm{~mL}$ concentration flask containing alcohol 37 (92.9 mg, $0.602 \mathrm{mmol}, 1$ equiv) in dry $\mathrm{CH}_{2} \mathrm{Cl}_{2}(6 \mathrm{~mL})$ was cooled to $0{ }^{\circ} \mathrm{C}$. Dess-Martin periodinane (DMP) $(0.332 \mathrm{~g}, 0.783 \mathrm{mmol}, 1.3$ equiv) was added, and the solution became a cloudy white. After $5 \mathrm{~min}$, the solution was warmed to rt. After $1 \mathrm{~h}$, a 1:1 mixture of sat aq $\mathrm{Na}_{2} \mathrm{~S}_{2} \mathrm{O}_{4}$ and sat aq $\mathrm{NaHCO}_{3}(12 \mathrm{~mL})$ was added to the crude mixture which was then stirred vigorously for $15 \mathrm{~min}$. The layers were separated, and the aqueous layer was extracted with $\mathrm{CH}_{2} \mathrm{Cl}_{2}(2 \times 12 \mathrm{~mL})$. The combined organics were washed with sat aq $\mathrm{NaHCO}_{3}(5 \mathrm{~mL})$, dried over $\mathrm{Na}_{2} \mathrm{SO}_{4}$, filtered, and concentrated in vacuo to give a light yellow oil with a white precipitate. The residue was suspended in $20 \% \mathrm{Et}_{2} \mathrm{O} /$ pentane $(5 \mathrm{~mL})$ and filtered through a small plug of Celite (additional $5 \mathrm{~mL}$ rinse). The solvent was removed in vacuo to aldehyde 38 (116 $\mathrm{mg}$, with DMP contaminants) as a light yellow oil (TLC: $R_{f}=0.82,15 \%$ EtOAc/hexanes, anisaldehyde stain) which was carried on without further purification. Due to the volatility of aldehyde 38, it should not be exposed to pressures lower than $5 \mathrm{~mm} \mathrm{Hg}:{ }^{1} \mathrm{H} \mathrm{NMR}\left(500 \mathrm{MHz}, \mathrm{CDCl}_{3}\right) \delta 9.57(\mathrm{t}, J=2.4 \mathrm{~Hz}, 1 \mathrm{H}), 5.42-5.34(\mathrm{~m}$, 2H), 5.32 (d, $J=10.2 \mathrm{~Hz}, 1 \mathrm{H}), 3.12(\mathrm{~d}, J=2.4 \mathrm{~Hz}, 2 \mathrm{H}), 2.95$ (ddq, $J=10.2,9.1,6.8 \mathrm{~Hz}, 1 \mathrm{H}), 1.76(\mathrm{~d}, J$ $=1.3 \mathrm{~Hz}, 3 \mathrm{H}), 1.64(\mathrm{~d}, J=5.6 \mathrm{~Hz}, 3 \mathrm{H}), 1.03(\mathrm{~d}, J=6.8 \mathrm{~Hz}, 3 \mathrm{H}) \mathrm{ppm} ;{ }^{13} \mathrm{C} \mathrm{NMR}\left(125 \mathrm{MHz}, \mathrm{CDCl}_{3}\right) \delta$ 199.5, 135.3, 134.8, 124.7, 123.3, 47.5, 35.8, 24.4, 21.4, 17.8 ppm; IR (film) 3022, 2966, 2927, 2870, $2717,1726,1450,1379,1238,1170,1120,1048,1017,970,857 \mathrm{~cm}^{-1}$.

(b) Horner-Wadsworth-Emmons Olefination. To a $10 \mathrm{~mL}$ concentration flask was added $\mathrm{NaH}$ (48 $\mathrm{mg}$ of a $60 \%$ dispersion in oil, $1.2 \mathrm{mmol}, 2.0$ equiv). The white solid was washed in dry pentanes $(3 \times 1 \mathrm{~mL})$ and suspended in dry THF $(0.4 \mathrm{~mL})$. To this suspension was added triethylphosphonoacetate $(0.251 \mathrm{~mL}$, $1.26 \mathrm{mmol}, 2.1$ equiv) dropwise via syringe, and the solid dissolved to give a light yellow solution. After $1 \mathrm{~h}$, the solution was cooled to $0{ }^{\circ} \mathrm{C}$, and unpurified aldehyde 38 (theoretical from above, $0.602 \mathrm{mmol}, 1$ equiv) was added via cannula in dry THF $(0.5 \mathrm{~mL})$, producing a bright yellow/orange solution. After 30 min, the solution was warmed to $\mathrm{rt}$ and after an additional $30 \mathrm{~min}$, sat aq $\mathrm{NH}_{4} \mathrm{Cl}(20 \mathrm{~mL})$ was added along with $\mathrm{Et}_{2} \mathrm{O}(20 \mathrm{~mL})$, and the layers were separated. The aqueous layer was further extracted with $\mathrm{Et}_{2} \mathrm{O}(2 \times 10 \mathrm{~mL})$, and the combined organic layers were dried over $\mathrm{Na}_{2} \mathrm{SO}_{4}$, filtered, and concentrated in vacuo to yield ethyl ester 39 (270 mg, with phosphate contaminants) as a clear, yellow oil (TLC: $\mathrm{R}_{\mathrm{f}}=$

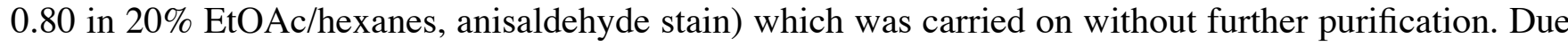
to the volatility of ester 39, it should not be exposed to pressures lower than $5 \mathrm{~mm} \mathrm{Hg}$ : ${ }^{1} \mathrm{H}$ NMR $(500$ $\left.\mathrm{MHz} \mathrm{CDCl}_{3}\right) \delta 6.90(\mathrm{dt}, J=15.6,6.7 \mathrm{~Hz}, 1 \mathrm{H}), 5.82(\mathrm{dt}, J=15.6,1.4 \mathrm{~Hz}, 1 \mathrm{H}), 5.41-5.31(\mathrm{~m}, 2 \mathrm{H}), 5.12$ $(\mathrm{d}, J=9.3 \mathrm{~Hz}, 1 \mathrm{H}), 4.18(\mathrm{q}, J=7.1 \mathrm{~Hz}, 2 \mathrm{H}), 2.95(\mathrm{dd}, J=6.7,2.0 \mathrm{~Hz}, 2 \mathrm{H}), 2.90(\mathrm{~m}, 1 \mathrm{H}), 1.68(\mathrm{~d}, J=$ $1.0 \mathrm{~Hz}, 3 \mathrm{H}), 1.63(\mathrm{~d}, J=5.0 \mathrm{~Hz}, 3 \mathrm{H}), 1.28(\mathrm{t}, J=7.1 \mathrm{~Hz}, 3 \mathrm{H}), 1.01(\mathrm{~d}, J=6.8 \mathrm{~Hz}, 3 \mathrm{H}) \mathrm{ppm} ;{ }^{13} \mathrm{C}$ NMR $\left(125 \mathrm{MHz}, \mathrm{CDCl}_{3}\right) \delta 166.6,146.4,135.7,132.3,129.7,123.0,122.0,60.2,35.6,35.0,23.5,21.4,17.9$, 14.3 ppm; IR (film) 3022, 2966, 2927, 2870, 2717, 1725, 1450, 1379, 1048, 970, $857 \mathrm{~cm}^{-1}$. 
(c) Reduction. To a $50 \mathrm{~mL}$ concentration flask containing ethyl ester $\mathbf{3 9}$ as an unpurified mixture (theoretical from above, $0.602 \mathrm{mmol}, 1$ equiv) in dry $\mathrm{CH}_{2} \mathrm{Cl}_{2}(8.6 \mathrm{~mL})$ at $0{ }^{\circ} \mathrm{C}$ was added DIBAL-H (1.99 $\mathrm{mL}$ of a $1.0 \mathrm{M}$ solution in $\mathrm{CH}_{2} \mathrm{Cl}_{2}, 1.99 \mathrm{mmol}, 3.3$ equiv) dropwise via syringe. Some gas evolution was evident. After $1 \mathrm{~h}$, half-saturated Rochelle's salt $(20 \mathrm{~mL})$ and $\mathrm{Et}_{2} \mathrm{O}(20 \mathrm{~mL})$ was added and the mixture stirred vigorously overnight and allowed to warm to rt. The layers were separated and the aqueous layer was further extracted with $\mathrm{Et}_{2} \mathrm{O}(3 \times 25 \mathrm{~mL})$. The combined organic layers were dried over $\mathrm{Na}_{2} \mathrm{SO}_{4}$, filtered, and concentrated in vacuo to yield a clear, yellow oil. The product was purified via automated silica column chromatography $\left(0 \rightarrow 15 \%\right.$ EtOAc/hexanes, $10 \mathrm{~g}$ column; TLC: $R_{\mathrm{f}}=0.28$ in $20 \% \mathrm{EtOAc} / \mathrm{hexanes,}$ CAM stain) to provide alcohol 40 (82.7 g, 76\% yield for three steps) as a clear, colorless oil. Due to the volatility of alcohol 40, it should not be exposed to pressures lower than $5 \mathrm{~mm}$ $\mathrm{Hg}$ for more than several hours. When left overnight at $0.5 \mathrm{~mm} \mathrm{Hg}$ for characterization purposes, partial product loss was observed: ${ }^{1} \mathrm{H}$ NMR $\left(500 \mathrm{MHz}, \mathrm{CDCl}_{3}\right) \delta$ 5.70-5.60 (m, 2H), 5.42-5.33 (m, 2H), 5.04 $(\mathrm{d}, J=9.4 \mathrm{~Hz}, 1 \mathrm{H}), 4.13-4.07(\mathrm{~m}, 2 \mathrm{H}), 3.05-2.97(\mathrm{~m}, 1 \mathrm{H}), 2.82-2.69(\mathrm{~m}, 2 \mathrm{H}), 1.67(\mathrm{~d}, J=1.4 \mathrm{~Hz}, 3 \mathrm{H})$, $1.64(\mathrm{~d}, J=3.9 \mathrm{~Hz}, 3 \mathrm{H}), 1.33(\mathrm{br} \mathrm{s}, 1 \mathrm{H}), 1.01(\mathrm{~d}, J=6.7 \mathrm{~Hz}, 3 \mathrm{H}) \mathrm{ppm} ;{ }^{13} \mathrm{C} \mathrm{NMR}\left(125 \mathrm{MHz}, \mathrm{CDCl}_{3}\right) \delta$ 136.1, 131.6, 130.9, 130.5, 129.9, 122.7, 63.7, 35.4, 35.0, 23.4, 21.5, 17.9 ppm; IR (film) 3325, 2963, 2870, 1449, 1377, 1042, 968, $868 \mathrm{~cm}^{-1} ;[\alpha]_{\mathrm{D}^{24}}=-80.6^{\circ}\left(c=1.00, \mathrm{CHCl}_{3}\right)$; HRMS (EI): Exact mass calcd for $\mathrm{C}_{12} \mathrm{H}_{20} \mathrm{O}[\mathrm{M}]^{+}$: 180.1514; Found: 180.1514; Anal calcd for $\mathrm{C}_{12} \mathrm{H}_{20} \mathrm{O}$ : C, 79.94\%, H, 11.18\%; Found: C, $79.72 \%, \mathrm{H}, 11.35 \%$.

Side Chain Allylic Bromide Coupling Fragment 5.
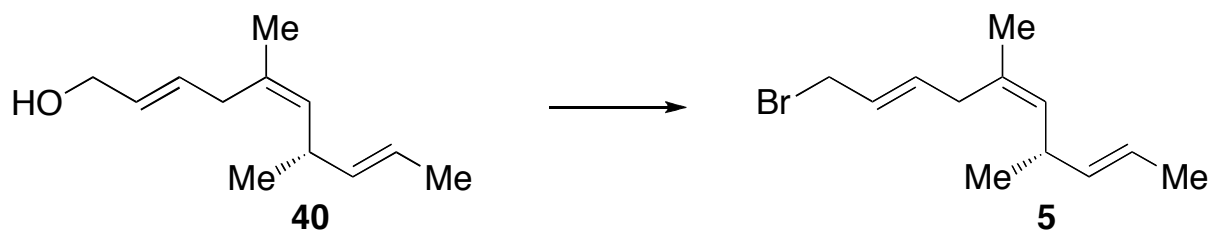

To a $25 \mathrm{~mL}$ concentration flask containing alcohol 40 (81.8 $\mathrm{mg}, 0.454 \mathrm{mmol}, 1$ equiv) in dry THF (2 $\mathrm{mL})$ was added $\mathrm{Et}_{3} \mathrm{~N}(0.253 \mathrm{~mL}, 1.82 \mathrm{mmol}, 4$ equiv $)$. The solution was cooled to $-40{ }^{\circ} \mathrm{C}$ and $\mathrm{MsCl}$ $(0.105 \mathrm{~mL}, 1.36 \mathrm{mmol}, 3$ equiv) was added dropwise. A white precipitate formed immediately. After 50 min at $40{ }^{\circ} \mathrm{C}$, the suspension was warmed to $0{ }^{\circ} \mathrm{C}$. After $30 \mathrm{~min}$, a solution of $\mathrm{LiBr}(0.394 \mathrm{~g}, 4.54 \mathrm{mmol}$, 10 equiv) in THF (4 mL) was added dropwise via cannula. After $30 \mathrm{~min}$, the flask was warmed to rt and stirred for an additional $30 \mathrm{~min}$. The reaction was quenched with ice-cold sat aq $\mathrm{NaHCO}_{3}(15 \mathrm{~mL})$ and diluted with pentane $(5 \mathrm{~mL})$. The layers were separated and the aqueous layer was further extracted with pentane $(3 \times 10 \mathrm{~mL})$. The combined organic layers were dried over $\mathrm{Na}_{2} \mathrm{SO}_{4}$, filtered, and concentrated in vacuo to yield a clear, yellow oil. The product was purified via automated silica column chromatography (pentane, 4 g column; TLC: $\mathrm{R}_{\mathrm{f}}=0.89$ in $20 \%$ EtOAc/hexanes, anisaldehyde stain) to provide bromide 5 (95.8 g, 87\% yield) as a clear, colorless oil. Due to the volatility of bromide $\mathbf{5}$, it should not be exposed to pressures lower than $5 \mathrm{~mm} \mathrm{Hg}$ for extended periods: ${ }^{1} \mathrm{H} \mathrm{NMR}\left(500 \mathrm{MHz}, \mathrm{CDCl}_{3}\right) \delta$ 5.75-5.67 (m, $2 \mathrm{H}), 5.42-5.32(\mathrm{~m}, 2 \mathrm{H}), 5.06(\mathrm{~d}, J=8.9 \mathrm{~Hz}, 1 \mathrm{H}), 4.00-3.92(\mathrm{~m}, 2 \mathrm{H}), 3.03-2.95(\mathrm{~m}, 1 \mathrm{H}), 2.82-2.74(\mathrm{~m}$, $2 \mathrm{H}), 1.66(\mathrm{~d}, J=1.3 \mathrm{~Hz}, 3 \mathrm{H}), 1.64(\mathrm{~d}, J=4.5 \mathrm{~Hz}, 3 \mathrm{H}), 1.01(\mathrm{~d}, J=6.8 \mathrm{~Hz}, 3 \mathrm{H}) \mathrm{ppm} ;{ }^{13} \mathrm{C}$ NMR $(125$ $\left.\mathrm{MHz}, \mathrm{CDCl}_{3}\right) \delta 136.0,133.8,131.4,131.0,127.2,122.8,35.4,34.9,33.3,23.5,21.5,18.0 \mathrm{ppm}$; IR (film) 2964, 2927, 2868, 156, 1438, 1377, 1203, 965, 854, $580 \mathrm{~cm}^{-1} ;[\alpha]_{\mathrm{D}^{24}}=-69.3^{\circ}\left(c=1.00, \mathrm{CHCl}_{3}\right)$; HRMS (EI): Exact mass calcd for $\mathrm{C}_{12} \mathrm{H}_{19} \mathrm{Br}[\mathrm{M}]^{+}$: 242.0670 ; Found: 242.0663. 
Thiazolidinethione Alcohol 51.

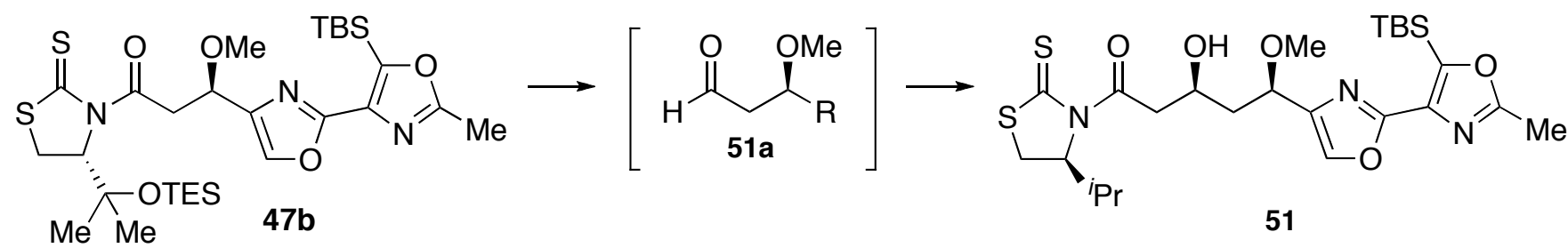

(a) Reduction. To a $10 \mathrm{~mL}$ flask containing bisoxazole methyl ether 47b (248 $\mathrm{mg}, 0.388 \mathrm{mmol}, 1$ equiv) in $\mathrm{CH}_{2} \mathrm{Cl}_{2}(1.5 \mathrm{~mL})$ under $\mathrm{N}_{2}$ at $-78{ }^{\circ} \mathrm{C}$ was added DIBAL-H $\left(0.581 \mathrm{~mL}\right.$ of a $1.0 \mathrm{M}$ solution in $\mathrm{CH}_{2} \mathrm{Cl}_{2}$, 1.5 equiv) dropwise via syringe. After stirring for $1 \mathrm{~h}$, the reaction was quenched by the addition of $\mathrm{MeOH}(7.5 \mathrm{~mL})$. Rochelle's salt (10 mL of a 7:5 mixture of saturated solution and water) was added and the biphasic mixture was warmed to $\mathrm{rt}$ and stirred rapidly for $45 \mathrm{~min}$. The layers were separated and the aqueous phase was extracted with $\mathrm{CH}_{2} \mathrm{Cl}_{2}(3 \times 25 \mathrm{~mL})$. The combined organic layers were dried over anhydrous $\mathrm{Na}_{2} \mathrm{SO}_{4}$, filtered, and concentrated in vacuo to give a colorless oil. This material was quickly subjected to flash chromatography $\left(\mathrm{CH}_{2} \mathrm{Cl}_{2}\right.$ until the auxiliary was eluted then $30 \% \mathrm{Et}_{2} \mathrm{O} / \mathrm{CH}_{2} \mathrm{Cl}_{2} ; \mathrm{TLC}$ : $\mathrm{R}_{\mathrm{f}}=0.09$ in $70 \% \mathrm{Et}_{2} \mathrm{O} /$ hexanes) to provide $(R)$-3-(2-(5-(tert-butyldimethylsilyl)-2-methyloxazol-4-yl) oxazol-4-yl)-3-methoxypropanal (51a) as a relatively unstable clear oil that was used immediately in the next step: ${ }^{1} \mathrm{H}$ NMR $\left(500 \mathrm{MHz}, \mathrm{CDCl}_{3}\right) \delta 9.83(\mathrm{~s}, 1 \mathrm{H}), 7.67(\mathrm{~s}, 1 \mathrm{H}), 4.81(\mathrm{~d}, J=7.6,4.7 \mathrm{~Hz}, 1 \mathrm{H}), 3.38$ (s, 3H), $3.03(\mathrm{dd}, J=16.9,7.7 \mathrm{~Hz}, 1 \mathrm{H}), 2.94(\mathrm{dd}, J=16.9,4.6 \mathrm{~Hz}, 1 \mathrm{H}), 2.55(\mathrm{~s}, 3 \mathrm{H}), 0.96(\mathrm{~s}, 9 \mathrm{H}), 0.34$ $(\mathrm{s}, 6 \mathrm{H}) \mathrm{ppm} ;{ }^{13} \mathrm{C} \mathrm{NMR}\left(125 \mathrm{MHz}, \mathrm{CDCl}_{3}\right) \delta$ 200.0, 165.1, 156.8, 154.8, 140.8, 138.7, 135.2, 71.3, 56.9, $48.0,26.3,17.4,13.6,-6.0 \mathrm{ppm}$.

(b) Aldol Reaction. To a $10 \mathrm{~mL}$ flask was added $\mathrm{Sn}(\mathrm{OTf})_{2}(0.353 \mathrm{~g}, 0.847 \mathrm{mmol}, 2.3 \mathrm{equiv})$ in a glove box. This tin compound was dissolved in $\mathrm{CH}_{2} \mathrm{Cl}_{2}(3.5 \mathrm{~mL})$, the flask was cooled to $-50{ }^{\circ} \mathrm{C}, N$-ethyl piperidine $(0.121 \mathrm{~mL}, 0.884 \mathrm{mmol}, 2.4$ equiv) was added dropwise via syringe, followed by $(S)-N-$ acetyl-4-iPr-thiazolidinethione (ent-44a, $0.122 \mathrm{~g}, 0.552 \mathrm{mmol}, 1.5 \mathrm{equiv})$. The solution was stirred at $50{ }^{\circ} \mathrm{C}$ for $4.5 \mathrm{~h}$ to form the tin enolate and then was cooled to $-110{ }^{\circ} \mathrm{C}$ in a liquid nitrogen/hexanes slurry before aldehyde 51a (theoretical from above, $0.388 \mathrm{mmol}, 1$ equiv) was added via cannula in $\mathrm{CH}_{2} \mathrm{Cl}_{2}(1.5 \mathrm{~mL})$. The solution was kept between -90 and $-100{ }^{\circ} \mathrm{C}$ for $2 \mathrm{~h}$ and was then warmed to $-78^{\circ}$ $\mathrm{C}$ over $30 \mathrm{~min}$ and allowed to stir for an additional $30 \mathrm{~min}$ at $-78{ }^{\circ} \mathrm{C}$. The reaction was quenched with pH 7 buffer and filtered with $\mathrm{CH}_{2} \mathrm{Cl}_{2}(25 \mathrm{~mL})$ through a plug of Celite into a separatory funnel containing sat aq $\mathrm{NaHCO}_{3}(15 \mathrm{~mL})$. The layers were separated and the aqueous layer was extracted with $\mathrm{CH}_{2} \mathrm{Cl}_{2}(2 \times 30 \mathrm{~mL})$. The combined organic phases were dried over anhydrous $\mathrm{Na}_{2} \mathrm{SO}_{4}$, filtered, and concentrated in vacuo to give a clear yellow oil. ${ }^{1} \mathrm{H}$ NMR integration of the unpurified product indicated a 3:1 diastereomer ratio. This material was purified via automated silica column chromatography $\left(40 \rightarrow 70 \%\right.$ EtOAc/hexanes, $110 \mathrm{~g}$ column; TLC: $\mathrm{R}_{\mathrm{f}}=0.28$ in $\left.70 \% \mathrm{EtOAc} / \mathrm{hexanes}\right)$ to provide diastereomerically pure alcohol $\mathbf{5 1}\left(214.2 \mathrm{mg}, 46 \%\right.$ yield over two steps) as a clear, yellow oil: ${ }^{1} \mathrm{H}$ NMR $\left(500 \mathrm{MHz} \mathrm{CDCl}_{3}\right) \delta 7.65(\mathrm{~s}, 1 \mathrm{H}), 5.15(\mathrm{dd}, J=7.0,6.8 \mathrm{~Hz}, 1 \mathrm{H}), 4.53(\mathrm{dd}, J=7.2,6.3 \mathrm{~Hz}, 1 \mathrm{H})$, 4.33-4.26 (m, 1H), 3.57-3.47 (m, 3H), 3.39-3.30 (m, 1H), $3.34(\mathrm{~s}, 3 \mathrm{H}), 3.03(\mathrm{~d}, J=11.1 \mathrm{~Hz}, 1 \mathrm{H}), 2.54$ (s, 3H), 2.42-2.31 (m, 1H), 2.20-2.12 (m, 1H), 2.11-2.03 (m, 1H). $1.06(\mathrm{~d}, J=6.7 \mathrm{~Hz}, 3 \mathrm{H}), 0.97(\mathrm{~d}, J=$ $7.3 \mathrm{~Hz}, 3 \mathrm{H}), 0.95$ (s, 9H), 0.38 (s, 6H) ppm; ${ }^{13} \mathrm{C} \mathrm{NMR}\left(125 \mathrm{MHz}, \mathrm{CDCl}_{3}\right) \delta$ 202.8, 172.2, 165.0, 156.7, $154.7,141.3,138.9,135.3,75.1,71.3,66.4,56.6,45.4,40.9,30.7,30.5,26.5,19.0,17.7,17.5,13.7,-5.9$ ppm; IR (film) 3420, 2958, 2930, 2855, 1696, 1616, 1583, 1469, 1363, 1314, 1157, 1094, 1036, 930, 844, 781, 730, $668 \mathrm{~cm}^{-1} ;[\alpha]_{\mathrm{D}}{ }^{25}=+212.1^{\circ}\left(c=1.03, \mathrm{CHCl}_{3}\right)$; HRMS (CI): Exact mass calcd for $\mathrm{C}_{25} \mathrm{H}_{40} \mathrm{~N}_{3} \mathrm{O}_{5} \mathrm{~S}_{2} \mathrm{Si}[\mathrm{M}+\mathrm{H}]^{+}:$554.2179; Found: 554.2181. 


\section{Glycal 54.}

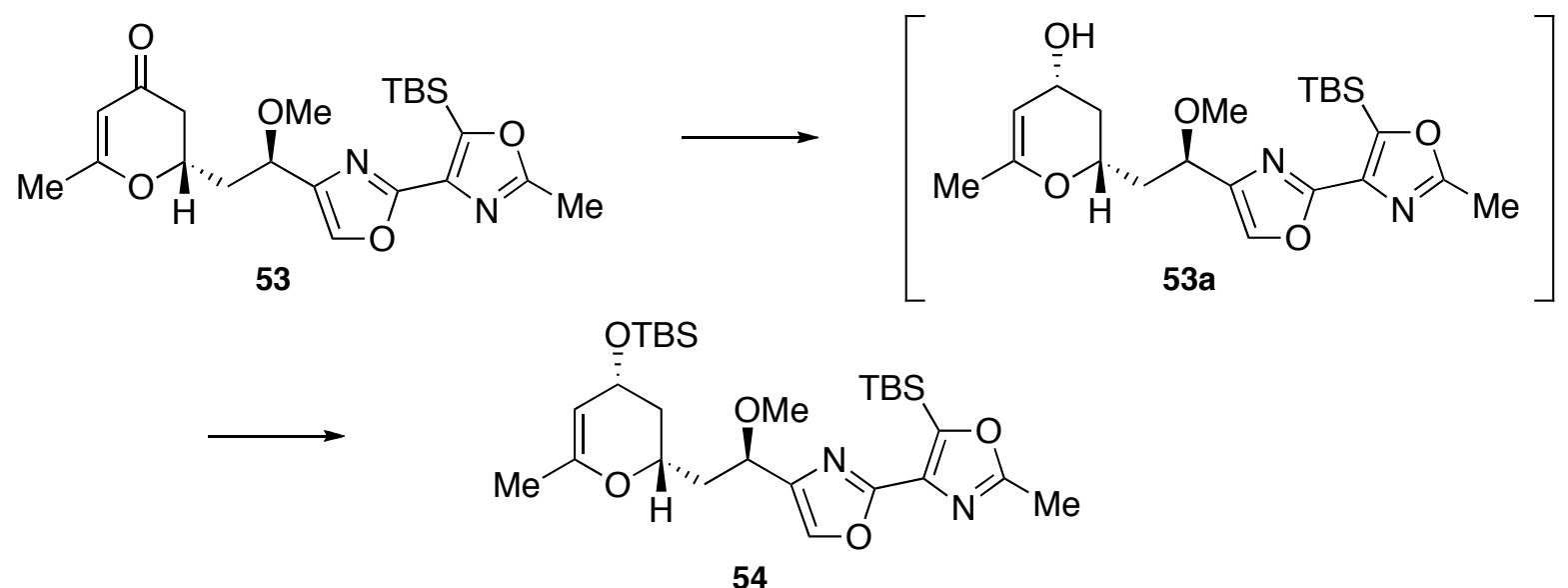

(a) Luche Reduction. To a $10 \mathrm{~mL}$ flask containing dihydropyranone $\mathbf{5 3}(218.7 \mathrm{mg}, 0.5056 \mathrm{mmol}, 1$ equiv) under an $\mathrm{Ar}$ atmosphere was added a solution of $\mathrm{CeCl}_{3} \bullet 7 \mathrm{H}_{2} \mathrm{O}(207 \mathrm{mg}, 0.556 \mathrm{mmol}, 1.1$ equiv $)$ in a 1:1 mixture of $\mathrm{THF} / \mathrm{MeOH}(7.2 \mathrm{~mL})$. The suspension was cooled to $-78{ }^{\circ} \mathrm{C}$ and $\mathrm{NaBH}_{4}(38.3 \mathrm{mg}, 1.01$ mmol, 2 equiv) was added as a solid in one portion. After $15 \mathrm{~min}$, the flask was allowed to warm to -40 ${ }^{\circ} \mathrm{C}$ over $1 \mathrm{~h}$.After an additional $45 \mathrm{~min}$ at $-40{ }^{\circ} \mathrm{C}$, the flask was recooled to $-78{ }^{\circ} \mathrm{C}$ and the reaction mixture was poured into $\mathrm{pH} 7$ buffer $(150 \mathrm{~mL})$ and was diluted with $\mathrm{Et}_{2} \mathrm{O}(50 \mathrm{~mL})$. The layers were separated and the aqueous phase was extracted with EtOAc $(4 \times 50 \mathrm{~mL})$ The combined organic layers were filtered through a plug of silica gel and concentrated in vacuo to give the corresponding allylic alcohol (53a) which was carried on immediately without any further purification: TLC: $\mathrm{R}_{\mathrm{f}}=0.36$ in $80 \%$ EtOAc/hexanes.

(b) TBS Protection. To a $5 \mathrm{~mL}$ flask containing allylic alcohol 53a (theoretical from above, 0.5056 mmol, 1 equiv) under an Ar atmosphere was added imidazole (103 mg, $1.52 \mathrm{mmol}, 3$ equiv) a catalytic amount of DMAP, and anhydrous DMF (2.0 mL). TBS-Cl (167.6 mg, $1.11 \mathrm{mmol}, 2.2$ equiv) was added and the reaction was stirred for $4 \mathrm{~h}$. The solution was diluted with brine $(50 \mathrm{~mL})$ and EtOAc $(50 \mathrm{~mL})$, the layers were separated, and the aqueous layer was extracted with EtOAc $(3 \times 50 \mathrm{~mL})$. The combined organics were washed with water $(2 \times 25 \mathrm{~mL})$, dried over anhydrous $\mathrm{Na}_{2} \mathrm{SO}_{4}$, filtered, and concentrated in vacuo. This material was purified via flash chromatography $\left(5 \rightarrow 9 \% \mathrm{Et}_{2} \mathrm{O} / \mathrm{CH}_{2} \mathrm{Cl}_{2}, \mathrm{TLC}: \mathrm{R}_{\mathrm{f}}=0.31\right.$ in $\left.10 \% \mathrm{Et}_{2} \mathrm{O} / \mathrm{CH}_{2} \mathrm{Cl}_{2}\right)$ to provide allylic TBS ether $54(177.0 \mathrm{mg}, 61 \%$ yield over two steps) as a clear colorless oil: ${ }^{1} \mathrm{H}$ NMR $\left(500 \mathrm{MHz}, \mathrm{CDCl}_{3}\right) \delta 7.63(\mathrm{~s}, 1 \mathrm{H}), 4.46(\mathrm{dd}, J=8.0,6.0 \mathrm{~Hz}, 1 \mathrm{H}), 4.42(\mathrm{~s}, 1 \mathrm{H})$, 4.35 (dd, $J=8.3,7.0 \mathrm{~Hz}, 1 \mathrm{H}), 3.86-3.78$ (m, 1H), 3.29 (s, 3H), 2.54 (s, 3H), 2.26 (ddd, $J=14.3,8.7,5.9$ $\mathrm{Hz}, 1 \mathrm{H}), 2.15$ (ddd, $J=12.7,8.2,4.3 \mathrm{~Hz}, 1 \mathrm{H}), 1.95$ (dd, $J=13.0,6.6 \mathrm{~Hz}, 1 \mathrm{H}), 1.69$ (s, 3H), 1.68-1.59 $(\mathrm{m}, 1 \mathrm{H}), 0.95$ (s, 9H), 0.88 (s, 9H), 0.39 (s, 3H), 0.38 (s, 3H), 0.06 (s, 6H) ppm; ${ }^{13} \mathrm{C}$ NMR $(125 \mathrm{MHz}$, $\left.\mathrm{CDCl}_{3}\right) \delta 165.1,156.6,154.7,152.0,141.2,139.1,135.9,101.3,72.6,71.5,64.3,56.3,39.5,37.6,26.5$, 25.9, 19.8, 18.2, 17.6, 13.8, -4.7, -5.9 ppm; IR (film) 2928, 2857, 1675, 1586, 1463, 1383, 1252, 1062, 837, 837, 780, $495 \mathrm{~cm}^{-1} ;[\alpha]_{\mathrm{D}}^{25}=+18.5^{\circ}\left(c=1.00, \mathrm{CHCl}_{3}\right)$; HRMS $(\mathrm{CI})$ : Exact mass calcd for $\mathrm{C}_{28} \mathrm{H}_{48} \mathrm{~N}_{2} \mathrm{O}_{5} \mathrm{Si}_{2} \mathrm{Li}[\mathrm{M}+\mathrm{Li}]^{+}:$555.3262; Found: 555.3267. 


\section{Preparation of Pyran/Bisoxazole Coupling Fragment 4a $(\mathrm{R}=\mathrm{TBS})$.}

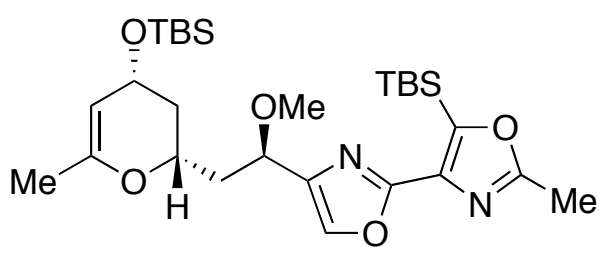

54

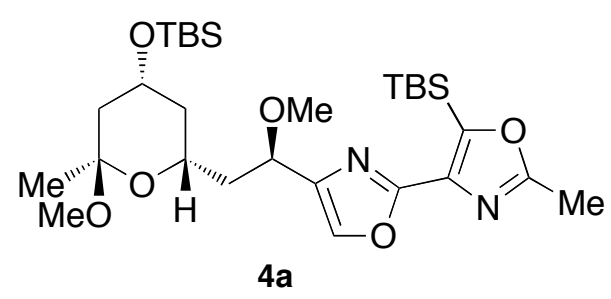

To a $5 \mathrm{~mL}$ flask containing TBS ether 54 (170.0 $\mathrm{mg}, 0.3097 \mathrm{mmol}, 1$ equiv) in benzene (10 $\mathrm{mL})$ under an argon atmosphere was added $\mathrm{MeOH}(0.376 \mathrm{~mL}, 30$ equiv) and trimethyl orthoformate $(0.170 \mathrm{~mL}, 5$ equiv). A spatula tip of PPTS was added in one portion and the reaction was stirred for $90 \mathrm{~min}$. The reaction was quenched with sat aq $\mathrm{NaHCO}_{3}(50 \mathrm{~mL})$ and diluted with EtOAc $(40 \mathrm{~mL})$. The layers were separated and the aqueous phase was further extracted with EtOAc $(3 \times 20 \mathrm{~mL})$. The combined organics were dried over anhydrous $\mathrm{Na}_{2} \mathrm{SO}_{4}$, filtered, and concentrated in vacuo. This material was purified via flash chromatography $\left(0 \rightarrow 40 \%\right.$ EtOAc/hexanes, TLC: $\mathrm{R}_{\mathrm{f}}=0.57$ in $40 \% \mathrm{EtOAc} /$ hexanes $)$ to provide mixed methyl acetal 4a (R = TBS, $144.4 \mathrm{mg}, 80 \%$ yield) as a clear colorless oil: ${ }^{1} \mathrm{H}$ NMR $(500 \mathrm{MHz}$, $\left.\mathrm{CDCl}_{3}\right) \delta 7.62(\mathrm{~s}, 1 \mathrm{H}), 4.45(\mathrm{dd}, J=7.7,6.4 \mathrm{~Hz}, 1 \mathrm{H}), 4.02-3.93(\mathrm{~m}, 1 \mathrm{H}), 3.51-3.42(\mathrm{~m}, 1 \mathrm{H}), 3.29(\mathrm{~s}$, $3 \mathrm{H}), 3.00(\mathrm{~s}, 3 \mathrm{H}), 2.54(\mathrm{~s}, 3 \mathrm{H}), 2.18-2.06(\mathrm{~m}, 2 \mathrm{H}), 1.95(\mathrm{dd}, J=12.8,4.5 \mathrm{~Hz}, 1 \mathrm{H}), 1.79(\mathrm{~d}, J=10.3 \mathrm{~Hz}$, $1 \mathrm{H}), 1.31(\mathrm{dd}, J=23.7,11.6 \mathrm{~Hz}, 1 \mathrm{H}), 1.30(\mathrm{~s}, 3 \mathrm{H}), 1.22(\mathrm{dd}, J=23.6,11.8 \mathrm{~Hz}, 1 \mathrm{H}), 0.95(\mathrm{~s}, 9 \mathrm{H}), 0.87$ $(\mathrm{s}, 9 \mathrm{H}), 0.39$ (s, 3H), $0.38(\mathrm{~s}, 3 \mathrm{H}), 0.04(\mathrm{~s}, 6 \mathrm{H}) \mathrm{ppm} ;{ }^{13} \mathrm{C} \mathrm{NMR}\left(125 \mathrm{MHz}, \mathrm{CDCl}_{3}\right) \delta$ 165.1, 156.6, 154.8, 141.4, 139.1, 135.9, 99.6, 72.7, 65.8, 65.1, 56.3, 47.6, 45.3, 41.0, 40.3, 26.5, 25.8, 23.7, 18.0, 17.6, 13.8, -4.6, -5.9 ppm; IR (film) 2929, 2857, 1612, 1586, 1463, 1377, 1319, 1251, 1192, 1084, 1034, 930, 914, $870,837,779,670,580,490 \mathrm{~cm}^{-1} ;[\alpha]_{\mathrm{D}^{25}}=-20.1^{\circ}\left(c=1.01, \mathrm{CHCl}_{3}\right)$; HRMS (CI): Exact mass calcd for $\mathrm{C}_{29} \mathrm{H}_{52} \mathrm{~N}_{2} \mathrm{O}_{6} \mathrm{Si}_{2} \mathrm{Li}[\mathrm{M}+\mathrm{Li}]^{+}:$587.3479; Found: 587.3523.

\section{Methylated Bisoxazole Dimethylacetal 24a}

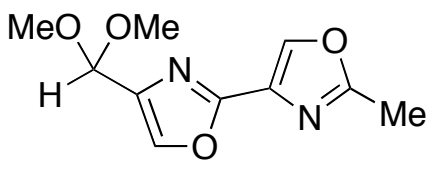

23

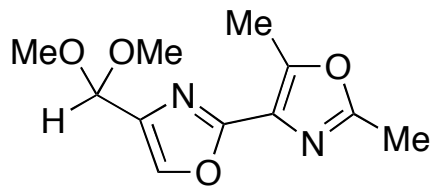

24a

To bisoxazole dimethylacetal $23\left(78.8 \mathrm{mg}, 0.351 \mathrm{mmol}, 1\right.$ equiv) in dry THF $(2.0 \mathrm{~mL})$ at $-78{ }^{\circ} \mathrm{C}$ was added $n$-BuLi (2.72 $\mathrm{M}$ in hexanes, $129 \mu \mathrm{L}, 0.351 \mathrm{mmol}, 1$ equiv). The reaction mixture gradually took on a bright yellow color. After stirring at $-78^{\circ} \mathrm{C}$ for $30 \mathrm{~min}$, MeI ( $24 \mu \mathrm{L}, 0.386 \mathrm{mmol}, 1.1$ equiv) was added dropwise. The reaction mixture was stirred at $-78{ }^{\circ} \mathrm{C}$ for $30 \mathrm{~min}$ and the color slowly faded from yellow to colorless. The reaction was quenched by the addition of sat. aq $\mathrm{NaHCO}_{3}(2.5 \mathrm{~mL})$ and the resulting suspension was warmed to rt and extracted with EtOAc. The combined organic layers were dried over a 1:1 mixture of $\mathrm{K}_{2} \mathrm{CO}_{3}$ and $\mathrm{Na}_{2} \mathrm{SO}_{4}$, filtered. and concentrated in vacuo to yield a colorless solid. Purification by silica gel chromatography $\left(0 \rightarrow 40 \%\right.$ EtOAc/hexanes; TLC: $R_{f}=0.60,100 \%$ ether UV) provided methylated bisoxazole dimethylacetal $\mathbf{2 4 a}(65.5 \mathrm{mg}, 78 \%)$ as a colorless, crystalline solid. Mp 97.0-98.5 ${ }^{\circ} \mathrm{C} ;{ }^{1} \mathrm{H}$ NMR $\left(500 \mathrm{MHz}, \mathrm{CDCl}_{3}\right) \delta 7.71(\mathrm{~s}, 1 \mathrm{H}), 5.47(\mathrm{~s}, 1 \mathrm{H}), 3.39(\mathrm{~s}, 6 \mathrm{H}), 2.64(\mathrm{~s}, 3 \mathrm{H})$, $2.47(\mathrm{~s}, 3 \mathrm{H}) \mathrm{ppm} ;{ }^{13} \mathrm{C} \mathrm{NMR}\left(125 \mathrm{MHz}, \mathrm{CDCl}_{3}\right) \delta 160.1,156.5,149.6,139.3,136.2,124.9,98.5,52.8$, 13.6, 11.5 ppm; IR (film) 2937, 1593, 1197, 1097, 1055, $980 \mathrm{~cm}^{-1}$; HRMS (EI): Exact mass calcd for $\mathrm{C}_{11} \mathrm{H}_{14} \mathrm{~N}_{2} \mathrm{O}_{4}[\mathrm{M}]^{+}:$238.0954; Found: 238.0950; Anal calcd for $\mathrm{C}_{11} \mathrm{H}_{14} \mathrm{~N}_{2} \mathrm{O}_{4}$ : C, 55.46\%, H, 5.92, $\mathrm{N}$, $11.76 \%$; Found: C, $55.57 \%, \mathrm{H}, 5.97 \%, \mathrm{~N}, 11.66 \%$. 


\section{Allylated Bisoxazole Dimethylacetal 27}
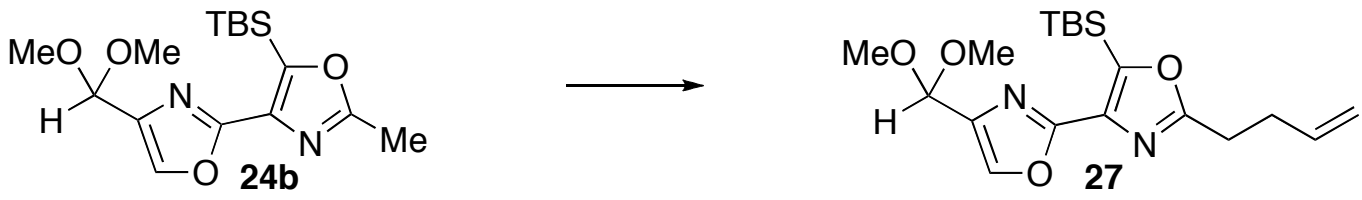

To as solution of diethylamine $\left(22.0 \mu \mathrm{L}, 0.213 \mathrm{mmol}, 1.5\right.$ equiv) in THF $(1 \mathrm{~mL})$ at $-78{ }^{\circ} \mathrm{C}$ was added $n$ $\operatorname{BuLi}(133 \mu \mathrm{L}$ of a $1.5 \mathrm{M}$ solution in hexanes, $0.199 \mathrm{mmol}, 1.4$ equiv). After stirring for $5 \mathrm{~min}$, warming to $0{ }^{\circ} \mathrm{C}$ for $10 \mathrm{~min}$, and then recooling to $-78{ }^{\circ} \mathrm{C}$, this solution was added via cannula to protected bisoxazole dimethyl acetal $\mathbf{2 4 b}\left(48.0 \mathrm{mg}, 0.142 \mathrm{mmol}, 1\right.$ equiv) in dry THF $(1 \mathrm{~mL})$ at $-78^{\circ} \mathrm{C}$. The reaction mixture immediately took on a bright red color and was stirred at $-78{ }^{\circ} \mathrm{C}$ for $30 \mathrm{~min}$. Allyl iodide (14.3 $\mu \mathrm{L}, 0.156 \mathrm{mmol}, 1.1$ equiv) was added dropwise, which caused a color change to light orange. After stirring at $-78{ }^{\circ} \mathrm{C}$ for $15 \mathrm{~min}$, sat aq $\mathrm{NaHCO}_{3}(2 \mathrm{~mL})$ was added and the reaction was warmed to rt. The resulting suspension was partitioned between $\mathrm{CH}_{2} \mathrm{Cl}_{2}(10 \mathrm{~mL})$ and sat aq $\mathrm{NaHCO}_{3}$ $(10 \mathrm{ml})$ and The aqueous layer was extracted with $\mathrm{CH}_{2} \mathrm{Cl}_{2}(2 \times 10 \mathrm{~mL})$. The combined organic layers were dried over a $1: 1$ mixture of $\mathrm{K}_{2} \mathrm{CO}_{3}$ and $\mathrm{Na}_{2} \mathrm{SO}_{4}$, filtered, and concentrated in vacuo to yield a colorless liquid. Purification by silica gel chromatography $\left(20 \% \mathrm{Et}_{2} \mathrm{O} /\right.$ hexanes; TLC: $\mathrm{R}_{\mathrm{f}}=0.77,70 \%$ $\mathrm{Et}_{2} \mathrm{O} /$ hexanes UV) provided allylated bisoxazole $27(47.0 \mathrm{mg}, 88 \%)$ as a clear colorless oil. ${ }^{1} \mathrm{H}$ NMR $\left(500 \mathrm{MHz}, \mathrm{CDCl}_{3}\right) \delta 7.70(\mathrm{~s}, 1 \mathrm{H}), 5.86(\mathrm{ddt}, J=17.1,10.2,6.5 \mathrm{~Hz}, 1 \mathrm{H}), 5.47(\mathrm{~s}, 1 \mathrm{H}), 5.08(\mathrm{dd}, J=17.1$, $1.6 \mathrm{~Hz}, 1 \mathrm{H}), 5.01(\mathrm{dd}, J=10.2,1.5 \mathrm{~Hz}, 1 \mathrm{H}), 3.37(\mathrm{~s}, 6 \mathrm{H}), 2.96(\mathrm{t}, J=7.9 \mathrm{~Hz}, 2 \mathrm{H}), 2.57(\mathrm{dt}, J=7.9,6.7$ $\mathrm{Hz}, 2 \mathrm{H}), 0.94$ (s, 9H), 0.38 (s, 6H) ppm; ${ }^{13} \mathrm{C} \mathrm{NMR}\left(125 \mathrm{MHz}, \mathrm{CDCl}_{3}\right) \delta$ 167.9, 156.6, 154.8, 139.6, 138.9, 136.5, 136.3, 115.9, 98.6, 52.8, 30.9, 27.5, 26.4, 17.6, -5.9 ppm; IR (film) 2931, 2858, 1612, 1580, 1470, 1251, 1391, 1193, 1103, $1062 \mathrm{~cm}^{-1}$; HRMS (EI): Exact mass calcd for $\mathrm{C}_{18} \mathrm{H}_{27} \mathrm{~N}_{2} \mathrm{O}_{3} \mathrm{Si}$ [M$\left.\mathrm{OCH}_{3}\right]^{+}:$347.1791; Found: 347.1795; Anal calcd for $\mathrm{C}_{19} \mathrm{H}_{30} \mathrm{~N}_{2} \mathrm{O}_{4} \mathrm{Si}: \mathrm{C}, 60.29 \%, \mathrm{H}, 7.99 \%$, N, 7.40\%; Found: C, $60.59 \%, \mathrm{H}, 8.02 \%, \mathrm{~N}, 7.37 \%$.

\section{Deprotected Allylated Bisoxazole 28}
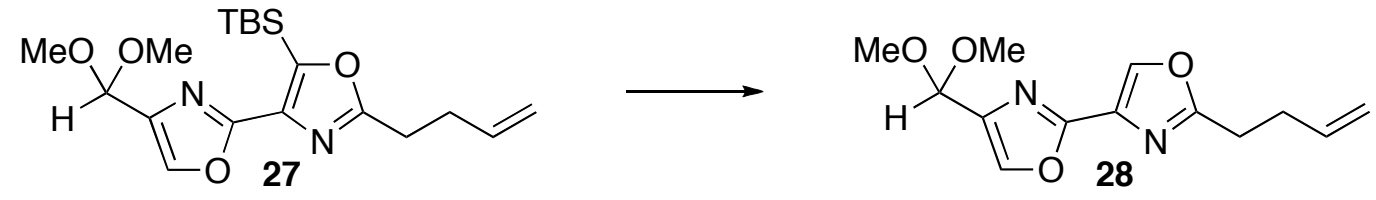

To alylated TBS-protected bisoxazle 27 ( $16 \mathrm{mg}, 0.042 \mathrm{mmol}, 1$ equiv) in dry THF ( $425 \mu \mathrm{L}$ ) at $0{ }^{\circ} \mathrm{C}$ was added tetrabutylammoium fluoride (TBAF) $(51 \mu \mathrm{L}$ of a $1.0 \mathrm{M}$ solution in THF, $0.051 \mathrm{mmol}, 1.2$ equiv). After stirring at $0{ }^{\circ} \mathrm{C}$ for $10 \mathrm{~min}$, the reaction was quenched by the addition of sat aq $\mathrm{NaHCO}_{3}(5 \mathrm{~mL})$ and $\mathrm{CH}_{2} \mathrm{Cl}_{2}(1 \mathrm{~mL})$. The mixture was partitioned between $\mathrm{CH}_{2} \mathrm{Cl}_{2}(5 \mathrm{~mL})$ and sat aq $\mathrm{NaHCO}_{3}(5 \mathrm{~mL})$ and the aqueous layer was extracted with $\mathrm{CH}_{2} \mathrm{Cl}_{2}(2 \times 5 \mathrm{~mL})$ The combined organic layers were dried over a 1:1 mixture of $\mathrm{K}_{2} \mathrm{CO}_{3}$ and $\mathrm{Na}_{2} \mathrm{SO}_{4}$, filtered, and concentrated in vacuo to yield a colorless liquid. Purification by silica gel chromatography $\left(1: 1: 1 \quad \mathrm{CH}_{2} \mathrm{Cl}_{2} / \mathrm{Et}_{2} \mathrm{O} /\right.$ hexanes; TLC: $\mathrm{R}_{\mathrm{f}}=0.53,70 \% \mathrm{Et}_{2} \mathrm{O} /$ hexanes, UV) provided deptotected allylated bisoxazole $28(10.0 \mathrm{mg}, 90 \%)$ as a clear colorless oil. ${ }^{1} \mathrm{H}$ NMR $\left(500 \mathrm{MHz}, \mathrm{CDCl}_{3}\right) \delta 8.18(\mathrm{~s}, 1 \mathrm{H}), 7.72(\mathrm{~s}, 1 \mathrm{H}), 5.86(\mathrm{ddt}, J=17.1,10.2,6.5 \mathrm{~Hz}, 1 \mathrm{H}), 5.48(\mathrm{~s}$, $1 \mathrm{H}), 5.09(\mathrm{dd}, J=17.1,1.6 \mathrm{~Hz}, 1 \mathrm{H}), 5.03(\mathrm{dd}, J=10.2,1.4 \mathrm{~Hz}, 1 \mathrm{H}), 3.39(\mathrm{~s}, 6 \mathrm{H}), 2.95(\mathrm{t}, J=7.4 \mathrm{~Hz}$, 2H), 2.58 (dt, $J=7.4,6.7 \mathrm{~Hz}, 2 \mathrm{H}) \mathrm{ppm} ;{ }^{13} \mathrm{C} \mathrm{NMR}\left(125 \mathrm{MHz}, \mathrm{CDCl}_{3}\right) \delta$ 165.4, 155.6, 139.5, 138.3, 136.8, 136.1, 130.3, 116.1, 98.3, 52.8, 30.7, 27.5 ppm; IR (film) 2939, 1103, 1059, 984, $916 \mathrm{~cm}^{-1}$; HRMS (EI): Exact mass calcd for $\mathrm{C}_{13} \mathrm{H}_{16} \mathrm{~N}_{2} \mathrm{O}_{4}$ [M]+: 264.1110; Found: 264.1114; Anal calcd for $\mathrm{C}_{13} \mathrm{H}_{16} \mathrm{~N}_{2} \mathrm{O}_{4}:$ C, 59.08\%, H, 6.10\%, N, 10.60\%; Found: C, 58.94\%, H, 6.16\%, N, 10.46\%. 


\section{Prenylated Bisoxazole 29}
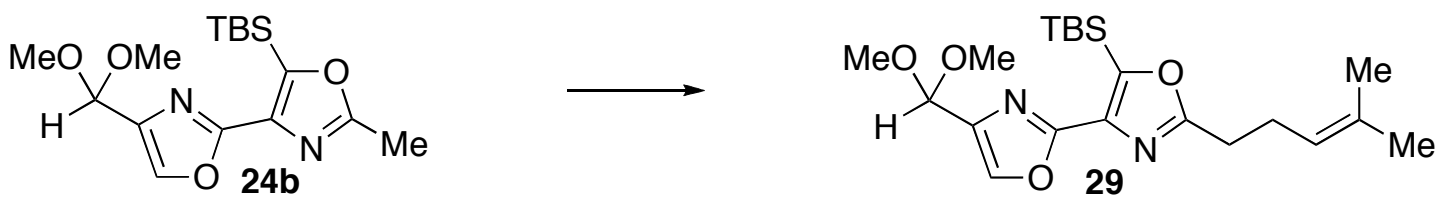

A $0.5 \mathrm{M}$ solution of $\mathrm{LiNEt}_{2}$ was prepared by addition of $n$-BuLi (1.00 mL of a $2.72 M$ solution in hexanes, $2.72 \mathrm{mmol})$ to diethylamine $(0.310 \mathrm{~mL}, 3.00 \mathrm{mmol})$ in $\mathrm{THF}(4.13 \mathrm{~mL})$ at $-78{ }^{\circ} \mathrm{C}$ under an atmosphere of argon. After $5 \mathrm{~min}$, the flask was warmed to $0{ }^{\circ} \mathrm{C}$. In a separate flask, TBS-protected bisoxazole dimethylacetal $\mathbf{2 4 b}\left(100.8 \mathrm{mg}, 0.298 \mathrm{mmol}, 1\right.$ equiv) in THF $(2.0 \mathrm{~mL})$ was cooled to $-78{ }^{\circ} \mathrm{C}$ under an atmosphere of argon and the $\mathrm{LiNEt}_{2}$ solution prepared above was added dropwise until a yellow color persisted (to remove any adventitious acid source-about 4 drops). After this zero point, $\mathrm{LiNEt}_{2}(0.893 \mathrm{~mL}$ of a $0.5 \mathrm{M}$ solution in THF, $0.447 \mathrm{mmol}, 1.5$ equiv) was added dropwise via gastight syringe. The reaction took on an bright orange/red color. After stirring at $-78{ }^{\circ} \mathrm{C}$ for 30 min, prenyl bromide $(39 \mu \mathrm{L}, 0.327 \mathrm{mmol}, 1.1$ equiv) was added dropwise causing the reaction to fade to light orange-yellow almost immediately. After $15 \mathrm{~min}$, the reaction was quenched with sat aq $\mathrm{NaHCO}_{3}(5$ $\mathrm{mL})$ and the color turned a very light yellow. The mixture was diluted with EtOAc $(5 \mathrm{~mL})$ and warmed to rt. The layers were separated and the aqueous phase was further extracted with EtOAc $(3 \times 5 \mathrm{~mL})$. The combined organics were dried over a 1:1 mixture of anhydrous $\mathrm{K}_{2} \mathrm{CO}_{3}$ and $\mathrm{Na}_{2} \mathrm{SO}_{4}$, filtered, and concentrated in vacuo. This material was purified via automated silica column chromatography $(0 \rightarrow 15 \%$ EtOAc/hexanes, TLC: $\mathrm{R}_{\mathrm{f}}=0.81$ in $70 \% \mathrm{Et}_{2} \mathrm{O} /$ hexanes) to provide prenylated bisoazole 29 (103.1 $\mathrm{mg}$, $91 \%$ yield) as a clear colorless oil ${ }^{1} \mathrm{H}$ NMR $\left(500 \mathrm{MHz}, \mathrm{CDCl}_{3}\right) \delta 7.70(\mathrm{~d}, J=1.0 \mathrm{~Hz}, 1 \mathrm{H}) 5.47(\mathrm{~d}, J=$ $0.8 \mathrm{~Hz}, 1 \mathrm{H}), 5.15(\mathrm{tt}, J=7.2,1.3 \mathrm{~Hz}, 1 \mathrm{H}), 3.37(\mathrm{~s}, 6 \mathrm{H}), 2.87(\mathrm{t}, J=7.4 \mathrm{~Hz}, 2 \mathrm{H}), 2.48(\mathrm{dt}, J=7.6,7.4 \mathrm{~Hz}$, $2 \mathrm{H}), 1.67(\mathrm{~s}, 3 \mathrm{H}), 1.60(\mathrm{~s}, 3 \mathrm{H}), 0.95,(\mathrm{~s}, 9 \mathrm{H}), 0.38(\mathrm{~s}, 6 \mathrm{H}) \mathrm{ppm} ;{ }^{13} \mathrm{C} \mathrm{NMR}\left(125 \mathrm{MHz}, \mathrm{CDCl}_{3}\right) \delta 168.3$, 156.7, 154.6, 139.6, 138.9, 136.5, 133.4, 122.2, 98.6, 52.8, 28.3, 26.4, 25.7, 25.6, 17.62, 17.58, -5.9 ppm; IR (film) 2954, 2930, 1469, 1251, 1104, 1062, 843, $124 \mathrm{~cm}^{-1}$; HRMS (EI): Exact mass calcd for $\mathrm{C}_{21} \mathrm{H}_{34} \mathrm{~N}_{2} \mathrm{O}_{4} \mathrm{Si}[\mathrm{M}]^{+}:$406.2288; Found: 406.2273; Anal calcd for $\mathrm{C}_{21} \mathrm{H}_{34} \mathrm{~N}_{2} \mathrm{O}_{4} \mathrm{Si}: \mathrm{C}, 62.03 \%, \mathrm{H}, 8.43 \%$, N, 6.89\%; Found: C, 62.17\%, H, 8.56\%, N, 6.94\%.

\section{Deprotected Prenylated Bisoxazole 30}
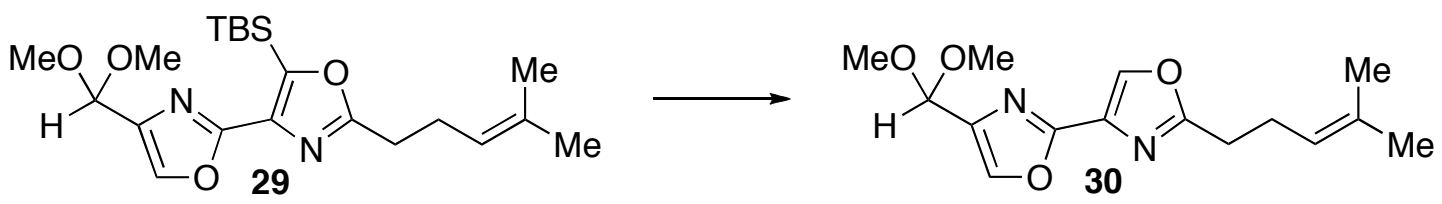

To prenylated TBS-protected bisoxazole $29\left(44.9 \mathrm{mg}, 0.110 \mathrm{mmol}, 1\right.$ equiv) in dry $\mathrm{THF}(2.0 \mathrm{~mL})$ at $0{ }^{\circ} \mathrm{C}$ was added tetrabutylammoium fluoride (TBAF) $(132 \mu \mathrm{L}$ of a $1.0 \mathrm{M}$ solution in THF, $0.133 \mathrm{mmol}, 1.2$ equiv). After stirring at $0{ }^{\circ} \mathrm{C}$ for $10 \mathrm{~min}$, the reaction was quenched by the addition of sat aq $\mathrm{NaHCO}_{3}(2$ $\mathrm{mL})$ and $\mathrm{CH}_{2} \mathrm{Cl}_{2}(2 \mathrm{~mL})$. The mixture was partitioned between $\mathrm{CH}_{2} \mathrm{Cl}_{2}(10 \mathrm{~mL})$ and sat aq $\mathrm{NaHCO}_{3}(10$ $\mathrm{mL})$ and the aqueous layer was extracted with $\mathrm{CH}_{2} \mathrm{Cl}_{2}(2 \times 10 \mathrm{~mL})$ The combined organic layers were dried over a 1:1 mixture of $\mathrm{K}_{2} \mathrm{CO}_{3}$ and $\mathrm{Na}_{2} \mathrm{SO}_{4}$, filtered, and concentrated in vacuo to yield a colorless liquid. Purification by silica gel chromatography $\left(1: 1: 2 \mathrm{CH}_{2} \mathrm{Cl}_{2} / \mathrm{Et}_{2} \mathrm{O} /\right.$ hexanes; TLC: $\mathrm{R}_{\mathrm{f}}=0.66,70 \%$ $\mathrm{Et}_{2} \mathrm{O} /$ hexanes, UV) provided deprotected prenylated bisoxazole $30(31.8 \mathrm{mg}, 99 \%)$ as a clear colorless oil. ${ }^{1} \mathrm{H}$ NMR $\left(500 \mathrm{MHz} \mathrm{CDCl}_{3}\right) \delta 8.18(\mathrm{~s}, 1 \mathrm{H}), 7.72(\mathrm{~d}, J=1.0 \mathrm{~Hz}, 1 \mathrm{H}), 5.48(\mathrm{~s}, 1 \mathrm{H}), 5.14(\mathrm{tt}, J=7.2$, $1.3 \mathrm{~Hz}, 1 \mathrm{H}), 3.39$ (s, 6H), $2.86(\mathrm{t}, J=7.4 \mathrm{~Hz}, 2 \mathrm{H}), 2.50(\mathrm{dt}, J=7.7,7.4 \mathrm{~Hz}, 2 \mathrm{H}), 1.68(\mathrm{~s}, 3 \mathrm{H}), 1.60$ (s, $3 \mathrm{H}) \mathrm{ppm} ;{ }^{13} \mathrm{C} \mathrm{NMR}\left(125 \mathrm{MHz}, \mathrm{CDCl}_{3}\right) \delta$ 165.8, 155.6, 139.5, 138.2, 136.8, 133.7, 130.2, 121.8, 98.3, 
52.8, 28.3, 25.6, 25.5, 17.6 ppm; IR (film) 2933, 1103, 1059, $984 \mathrm{~cm}^{-1}$; HRMS (EI): Exact mass calcd for $\mathrm{C}_{15} \mathrm{H}_{20} \mathrm{~N}_{2} \mathrm{O}_{4}[\mathrm{M}]^{+}: 292.1423$; Found: 292.1392; Anal calcd for $\mathrm{C}_{15} \mathrm{H}_{20} \mathrm{~N}_{2} \mathrm{O}_{4}: \mathrm{C}, 61.63 \%, \mathrm{H}, 6.90 \%, \mathrm{~N}$, 9.58\%; Found: C, $61.38 \%, \mathrm{H}, 7.06 \%, \mathrm{~N}, 9.36 \%$.

\section{Trienyl Bisozaxole 41}
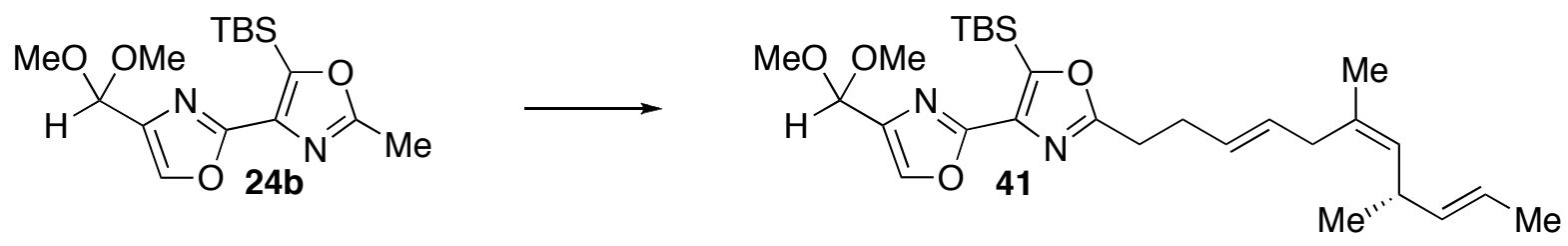

To as solution of diethylamine ( $59 \mu \mathrm{L}, 0.568 \mathrm{mmol}, 2.1$ equiv) in THF $(1.5 \mathrm{~mL})$ at $-78{ }^{\circ} \mathrm{C}$ was added $n$ BuLi $(340 \mu \mathrm{L}$ of a $1.59 \mathrm{M}$ solution in hexanes, $0.541 \mathrm{mmol}, 2.0$ equiv). After stirring for $5 \mathrm{~min}$, warming to $0{ }^{\circ} \mathrm{C}$ for $10 \mathrm{~min}$, and then recooling to $-78{ }^{\circ} \mathrm{C}$, this solution was added via cannula to protected bisoxazole dimethyl acetal $\mathbf{2 4 b}\left(93.1 \mathrm{mg}, 0.271 \mathrm{mmol}, 1\right.$ equiv) in dry THF $(1.5 \mathrm{~mL})$ at $-78^{\circ}$ C. The reaction mixture immediately took on a bright red color and was stirred at $-78{ }^{\circ} \mathrm{C}$ for $30 \mathrm{~min}$. Side chain allylic bromide 7 (106 mg, $0.436 \mathrm{mmol}, 1.5$ equiv) in THF ( $1 \mathrm{~mL})$ was added dropwise via cannula, which caused a color change to light orange. After stirring at $-78{ }^{\circ} \mathrm{C}$ for $15 \mathrm{~min}$, sat aq $\mathrm{NaHCO}_{3}(4 \mathrm{~mL})$ was added and the reaction was warmed to rt. The resulting suspension was partitioned between $\mathrm{CH}_{2} \mathrm{Cl}_{2}(15 \mathrm{~mL})$ and sat aq $\mathrm{NaHCO}_{3}(15 \mathrm{ml})$ and The aqueous layer was extracted with $\mathrm{CH}_{2} \mathrm{Cl}_{2}$ $(2 \times 15 \mathrm{~mL})$. The combined organic layers were dried over $\mathrm{Na}_{2} \mathrm{SO}_{4}$, filtered, and concentrated in vacuo to yield a clear yellow liquid. Purification by silica gel chromatography $\left(25 \% \mathrm{Et}_{2} \mathrm{O} /\right.$ hexanes; TLC: $\mathrm{R}_{\mathrm{f}}=$ $0.81,70 \% \mathrm{Et}_{2} \mathrm{O} /$ hexanes $\left.\mathrm{UV}\right)$ provided trienyl bisoxazole $41(106.7 \mathrm{mg}, 79 \%)$ as a clear colorless oil. ${ }^{1} \mathrm{H}$ NMR (500 MHz, CDCl $) \delta 7.70(\mathrm{~d}, J=0.8 \mathrm{~Hz}, 1 \mathrm{H}), 5.50-5.39(\mathrm{~m}, 2 \mathrm{H}), 5.47(\mathrm{~s}, 1 \mathrm{H}), 5.35-5.33(\mathrm{~m}, 2 \mathrm{H})$, $4.99(\mathrm{~d}, J=9.2 \mathrm{~Hz}, 1 \mathrm{H}), 3.37(\mathrm{~s}, 6 \mathrm{H}), 3.03-2.95(\mathrm{~m}, 1 \mathrm{H}), 2.91(\mathrm{t}, J=7.4 \mathrm{~Hz}, 2 \mathrm{H}), 2.71(\mathrm{dd}, J=14.4,6.2$ $\mathrm{Hz}, 1 \mathrm{H}), 2.65(\mathrm{dd}, J=14.4,6.2 \mathrm{~Hz}, 1 \mathrm{H}), 2.51(\mathrm{dt}, J=7.4,7.4 \mathrm{~Hz}, 2 \mathrm{H}), 1.63(\mathrm{~d}, J=4.7 \mathrm{~Hz}, 3 \mathrm{H}), 1.60(\mathrm{~d}$, $J=1.2 \mathrm{~Hz}, 3 \mathrm{H}), 0.99(\mathrm{~d}, J=6.7 \mathrm{~Hz}, 3 \mathrm{H}), 0.95(\mathrm{~s}, 9 \mathrm{H}), 0.38(\mathrm{~s}, 6 \mathrm{H}) \mathrm{ppm} ;{ }^{13} \mathrm{C}$ NMR $\left(125 \mathrm{MHz}, \mathrm{CDCl}_{3}\right) \delta$ 166.0, 156.6, 154.7, 139.6, 138.9, 136.5, 136.2, 132.1, 130.4, 129.5, 128.8, 122.5, 98.6, 52.8, 35.3, 35.2, 29.9, 28.2, 26.5, 23.3, 21.4, 17.9, 17.6, -5.9 ppm; IR (film) 2957, 2930, 2858, 1612, 1579, 1464, 1448, 1376, 1318, 1251, 1193, 1161, 1104, 1063, 1005, 970, 929, 910, 843, 824, 812, 782, 748, 688, 582, 456 $\mathrm{cm}^{-1}$; HRMS (EI): Exact mass calcd for $\mathrm{C}_{28} \mathrm{H}_{44} \mathrm{~N}_{2} \mathrm{O}_{4} \mathrm{Si}$ [M] ${ }^{+}:$500.3070; Found: 500.3080; Anal calcd for $\mathrm{C}_{28} \mathrm{H}_{44} \mathrm{~N}_{2} \mathrm{O}_{4} \mathrm{Si}$ : C, $67.16 \%, \mathrm{H}, 8.86 \%$, N, 5.59\%; Found: C, 67.43\%, H, 8.94\%, N, 5.40\%.

\section{Deprotected Trienyl Bisoxazole 42}

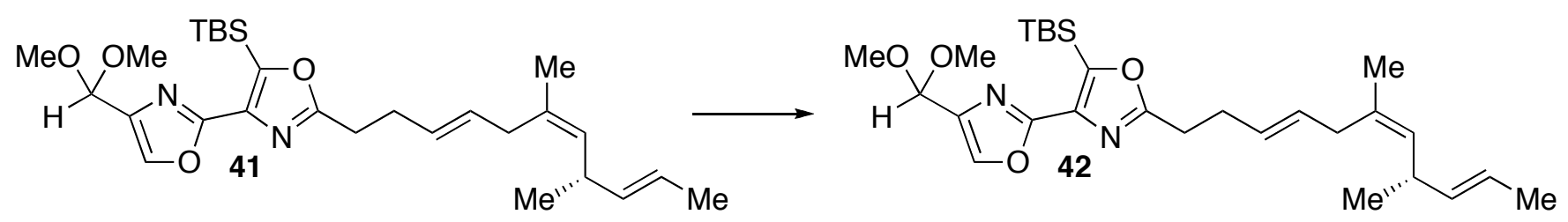

To TBS-protected trienyl bisoxazole $41\left(54.4 \mathrm{mg}, 0.109 \mathrm{mmol}, 1\right.$ equiv) in dry THF $(2.2 \mathrm{~mL})$ at $0{ }^{\circ} \mathrm{C}$ was added tetrabutylammoium fluoride (TBAF) $(130 \mu \mathrm{L}$ of a $1.0 \mathrm{M}$ solution in THF, $0.130 \mathrm{mmol}, 1.2$ equiv). After stirring at $0{ }^{\circ} \mathrm{C}$ for $10 \mathrm{~min}$, the reaction was quenched by the addition of sat aq $\mathrm{NaHCO}_{3}(2$ $\mathrm{mL}$ ). The mixture was partitioned between $\mathrm{CH}_{2} \mathrm{Cl}_{2}(10 \mathrm{~mL})$ and sat aq $\mathrm{NaHCO}_{3}(10 \mathrm{~mL})$ and the aqueous layer was extracted with $\mathrm{CH}_{2} \mathrm{Cl}_{2}(2 \times 10 \mathrm{~mL})$ The combined organic layers were dried over $\mathrm{Na}_{2} \mathrm{SO}_{4}$, filtered, and concentrated in vacuo to yield an orange liquid. Purification by silica gel 
chromatography (1:1:2 $\mathrm{CH}_{2} \mathrm{Cl}_{2} / \mathrm{Et}_{2} \mathrm{O} /$ hexanes; TLC: $\mathrm{R}_{\mathrm{f}}=0.66,70 \% \mathrm{Et}_{2} \mathrm{O} /$ hexanes, UV) provided deprotected trienyl bisoxazole $42(39.8 \mathrm{mg}, 95 \%)$ as a clear colorless oil. ${ }^{1} \mathrm{H}$ NMR $\left(500 \mathrm{MHz}, \mathrm{CDCl}_{3}\right) \delta$ ppm; ${ }^{13} \mathrm{C}$ NMR $\left(125 \mathrm{MHz}, \mathrm{CDCl}_{3}\right) \delta 8.16(\mathrm{~s}, 1 \mathrm{H}), 7.71(\mathrm{~d}, J=1.0 \mathrm{~Hz}, 1 \mathrm{H}), 5.49-5.41(\mathrm{~m}, 2 \mathrm{H}), 5.47(\mathrm{~s}$, $1 \mathrm{H}), 5.37-5.30(\mathrm{~m}, 2 \mathrm{H}), 4.98(\mathrm{~d}, J=9.2 \mathrm{~Hz}, 1 \mathrm{H}), 3.40(\mathrm{~s}, 3 \mathrm{H}), 3.02-2.93(\mathrm{~m}, 1 \mathrm{H}), 2.89(\mathrm{t}, J=7.4, \mathrm{~Hz}$, 2H), $2.70(\mathrm{dd}, J=14.6,6.2 \mathrm{~Hz}, 1 \mathrm{H}), 2.65(\mathrm{dd}, J=14.6,6.2 \mathrm{~Hz}, 1 \mathrm{H}), 2.51(\mathrm{dt}, J=7.4,7.4 \mathrm{~Hz}, 2 \mathrm{H}), 1.62$ $(\mathrm{d}, J=4.9 \mathrm{~Hz}, 3 \mathrm{H}), 1.60(\mathrm{~d}, J=1.2 \mathrm{~Hz}, 3 \mathrm{H}), 0.97$ (d, $J=6.9 \mathrm{~Hz}, 3 \mathrm{H}) \mathrm{ppm}$; IR (film) 3135, 2962, 2832, 2730, 1635, 1579, 1829, 1501, 1448, 1377, 1310, 1266, 1193, 1158, 1102, 1061, 969, 917, 854, 781, 734, $653 \mathrm{~cm}^{-1}$; HRMS (EI): Exact mass calcd for $\mathrm{C}_{22} \mathrm{H}_{30} \mathrm{~N}_{2} \mathrm{O}_{4}[\mathrm{M}]^{+}:$386.2206; Found: 386.2190; Anal calcd for $\mathrm{C}_{22} \mathrm{H}_{30} \mathrm{~N}_{2} \mathrm{O}_{4}$ : C, 68.37\%, H, 7.82\%, N, 7.25\%; Found: C, 68.52\%, H, 8.04\%, N, 7.24\%.

\section{General procedure for $\mathrm{TiCl}_{4} / \mathrm{Hünig}$ 's base aldol-type reactions with acetals}

To the $\mathrm{N}$-acetyl-thiazolidinethione (1.3 equiv) in $\mathrm{CH}_{2} \mathrm{Cl}_{2}(0.2 \mathrm{M})$ under argon atmosphere at $0{ }^{\circ} \mathrm{C}$ was added $\mathrm{TiCl}_{4}$ (1.3 equiv) dropwise via syringe. After $10 \mathrm{~min}$, the orange solution was cooled to $-78{ }^{\circ} \mathrm{C}$ and $(i-\operatorname{Pr})_{2} \mathrm{NEt}$ (1.3 equiv) was added via syringe and the solution turned a characteristic blood-red color. After stirring for $30 \mathrm{~min}$, the solution was warmed to $-50{ }^{\circ} \mathrm{C}$ and stirred for $2 \mathrm{~h}$. The dimethyl acetal (1 equiv) was added dropwise via cannula in $\mathrm{CH}_{2} \mathrm{Cl}_{2}$ (approx $0.3 \mathrm{M}$ ) followed by the dropwise addition of $\mathrm{BF}_{3} \bullet \mathrm{OEt}_{2}\left(1.3\right.$ equiv). The solution was stirred at $-78^{\circ} \mathrm{C}$ for $2 \mathrm{~h}$. The reaction was quenched by pouring into a rapidly stirring 1:1 mixture of $\mathrm{CH}_{2} \mathrm{Cl}_{2}$ and half-sat $\mathrm{NH}_{4} \mathrm{Cl}$. The layers were separated and the aqueous layer was re-extracted with $\mathrm{CH}_{2} \mathrm{Cl}_{2}$ and the combined organic layers were dried over anhydrous $\mathrm{Na}_{2} \mathrm{SO}_{4}$, filtered, and concentrated in vacuo. ${ }^{1} \mathrm{H}$ NMR integration of the unpurified product was used to determine the diastereomer ratio. Silica column chromatography provided diastereomerically pure methyl ether.

\section{General procedure for $\mathrm{PhBCl}_{2} /$ sparteine aldol-type reactions with acetals}

To the $N$-acetyl-thiazolidinethione $\left(1.3\right.$ equiv) in $\mathrm{CH}_{2} \mathrm{Cl}_{2}(0.2 \mathrm{M})$ under argon atmosphere at $0{ }^{\circ} \mathrm{C}$ was added $\mathrm{PhBCl}_{2}$ (1.3 equiv) dropwise via syringe. After $10 \mathrm{~min}$, sparteine (2.6 equiv) was added via syringe and the resulting solution was warmed to rt and stirred for $30 \mathrm{~min}$. The solution was cooled to $78{ }^{\circ} \mathrm{C}$ and the dimethyl acetal (1 equiv) was added dropwise via cannula in $\mathrm{CH}_{2} \mathrm{Cl}_{2}$ (approx $0.3 \mathrm{M}$ ) followed by the dropwise addition of $\mathrm{BF}_{3}{ }^{\bullet} \mathrm{OEt}_{2}\left(1.1\right.$ equiv). The solution was stirred at $-78{ }^{\circ} \mathrm{C}$ for $1.5 \mathrm{~h}$ and then was warmed to rt over $2 \mathrm{~h}$. After stirring at $\mathrm{rt}$ an additional $1 \mathrm{~h}$, the reaction was quenched with sat aq $\mathrm{NH}_{4} \mathrm{Cl}$. The contents of the flask were transferred to a separatory funnel containing a 4:1 mixture of hexanes: $\mathrm{CH}_{2} \mathrm{Cl}_{2}$. The layers were separated and the aqueous layer was re-extracted with $\mathrm{CH}_{2} \mathrm{Cl}_{2}$ and the combined organic layers were washed with brine, dried over anhydrous $\mathrm{Na}_{2} \mathrm{SO}_{4}$, filtered, and concentrated in vacuo. ${ }^{1} \mathrm{H}$ NMR integration of the unpurified product was used to determine the diastereomer ratio. Silica column chromatography provided diastereomerically pure methyl ether. 


\section{Methyl Ether 45a}

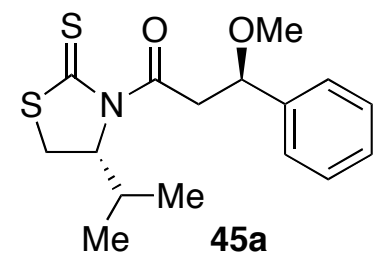

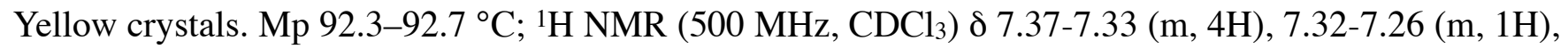
$5.00(\mathrm{ddd}, J=7.7,6.0,1.5 \mathrm{~Hz}, 1 \mathrm{H}), 4.71(\mathrm{dd}, J=7.6,5.7 \mathrm{~Hz}, 1 \mathrm{H}), 3.86(\mathrm{dd}, J=16.6,7.6 \mathrm{~Hz}, 1 \mathrm{H}), 3.69$ $(\mathrm{dd}, J=16.6,5.7 \mathrm{~Hz}, 1 \mathrm{H}), 3.30(\mathrm{dd}, J=11.4,8.1 \mathrm{~Hz}, 1 \mathrm{H}), 3.21(\mathrm{~s}, 3 \mathrm{H}), 2.96(\mathrm{dd}, J=11.4,1.5 \mathrm{~Hz}, 1 \mathrm{H})$, 2.40-2.31 (m, 1H), $1.04(\mathrm{~d}, J=6.9 \mathrm{~Hz}, 3 \mathrm{H}), 0.97(\mathrm{~d}, J=7.1,3 \mathrm{H}) \mathrm{ppm} ;{ }^{13} \mathrm{C} \mathrm{NMR}\left(125 \mathrm{MHz}, \mathrm{CDCl}_{3}\right) \delta$ 202.7, 171.0, 140.4, 128.4, 127.9, 126.9, 79.9, 71.7, 56.5, 46.1, 30.5, 30.2, 19.0, 17.4 ppm; IR (film) 2964, 2933, 2891, 2821, 1698, 1467, 1364, 1306, 1255, 1207, 1156, 1096, 1038, 1006, 987, 895, 701 $\mathrm{cm}^{-1} ;[\alpha]_{\mathrm{D}^{24}}=-214.2^{\circ}\left(c=1.00, \mathrm{CHCl}_{3}\right)$; HRMS (EI): Exact mass calcd for $\mathrm{C}_{16} \mathrm{H}_{21} \mathrm{NO}_{2} \mathrm{~S}_{2}[\mathrm{M}]^{+}$: 323.1014; Found: 323.0997.

\section{Methyl Ether 46a}

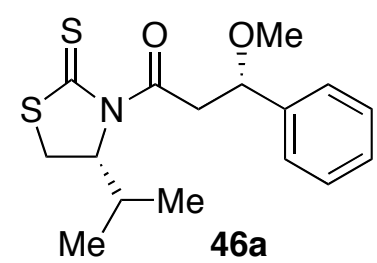

Clear yellow oil: ${ }^{1} \mathrm{H}$ NMR $\left(500 \mathrm{MHz}, \mathrm{CDCl}_{3}\right) \delta$ 7.39-7.33 (m, 4H), 7.33-7.28 (m, 1H), $5.16(\mathrm{dd}, J=6.9$, $6.9 \mathrm{~Hz}, 1 \mathrm{H}), 4.81(\mathrm{dd}, J=9.7,3.0 \mathrm{~Hz}, 1 \mathrm{H}), 3.76(\mathrm{dd}, J=17.1,9.7 \mathrm{~Hz}, 1 \mathrm{H}), 3.53(\mathrm{dd}, J=11.4,8.1 \mathrm{~Hz}$, $1 \mathrm{H}), 3.46(\mathrm{dd}, J=17.1,3.1 \mathrm{~Hz}, 1 \mathrm{H}), 3.22(\mathrm{~s}, 3 \mathrm{H}), 3.03(\mathrm{~d}, J=11.4,1 \mathrm{H}), 2.41-2.25(\mathrm{~m}, 1 \mathrm{H}), 1.04(\mathrm{~d}, J=$ $6.7 \mathrm{~Hz}, 3 \mathrm{H}), 0.96(\mathrm{~d}, J=6.9,3 \mathrm{H}) \mathrm{ppm} ;{ }^{13} \mathrm{C} \mathrm{NMR}\left(125 \mathrm{MHz}, \mathrm{CDCl}_{3}\right) \delta 202.8,171.0,140.5,128.5$, 128.0, 126.7, 79.5, 71.7, 56.7, 46.6, 30.8, 30.7, 19.0, 17.7 ppm; IR (film) 3029, 2961, 2930, 2822, 1678, $1455,1365,1313,1275,1161,1096,1039,762,701 \mathrm{~cm}^{-1} ;[\alpha]_{\mathrm{D}^{24}}=-266.0^{\circ}\left(c=1.34, \mathrm{CHCl}_{3}\right)$; HRMS (EI): Exact mass calcd for $\mathrm{C}_{16} \mathrm{H}_{21} \mathrm{NO}_{2} \mathrm{~S}_{2}[\mathrm{M}]^{+}:$323.1014; Found: 323.1053 .

\section{Methyl Ether 45b}

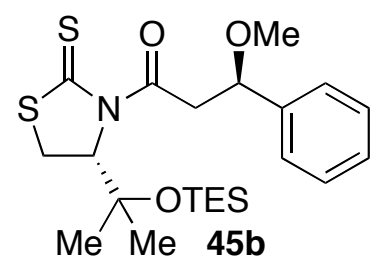

Clear yellow oil: ${ }^{1} \mathrm{H}$ NMR $\left(500 \mathrm{MHz}, \mathrm{CDCl}_{3}\right) \delta$ 7.37-7.33 (m, 4H), 7.32-7.27 (m, 1H), $5.19(\mathrm{dd}, J=8.1$, $1.0 \mathrm{~Hz}, 1 \mathrm{H}), 4.72(\mathrm{dd}, J=7.8,5.5 \mathrm{~Hz}, 1 \mathrm{H}), 3.90(\mathrm{dd}, J=16.7,7.8 \mathrm{~Hz}, 1 \mathrm{H}), 3.65(\mathrm{dd}, J=16.7,5.5 \mathrm{~Hz}$, $1 \mathrm{H}), 3.35(\mathrm{dd}, J=11.4,1.1 \mathrm{~Hz}, 1 \mathrm{H}), 3.30(\mathrm{dd}, J=11.4,8.2 \mathrm{~Hz}, 1 \mathrm{H}), 3.21(\mathrm{~s}, 3 \mathrm{H}), 1.30(\mathrm{~s}, 3 \mathrm{H}), 1.29(\mathrm{~s}$, $3 \mathrm{H}), 0.95(\mathrm{t}, J=7.9 \mathrm{~Hz}, 6 \mathrm{H}), 0.61(\mathrm{q}, J=7.9 \mathrm{~Hz}, 4 \mathrm{H}) \mathrm{ppm} ;{ }^{13} \mathrm{C}$ NMR $\left(125 \mathrm{MHz}, \mathrm{CDCl}_{3}\right) \delta 205.3$, 170.5, 140.6, 128.4, 127.9, 127.0, 79.9 76.7, 72.6, 56.6, 45.8, 30.1, 28.1, 26.1, 7.0, 6.5 ppm; IR (film) 2955, 2937, 2876, 2823, 1736, 1700, 1456, 1369, 1312, 1272, 1242, 1169, 1150, 1102, 1036, 745, 724, 
$701 \mathrm{~cm}^{-1} ;[\alpha]_{\mathrm{D}^{24}}=-74.5^{\circ}\left(c=1.00, \mathrm{CHCl}_{3}\right)$; HRMS (EI): Exact mass calcd for $\mathrm{C}_{22} \mathrm{H}_{35} \mathrm{NO}_{3} \mathrm{~S}_{2} \mathrm{Si}[\mathrm{M}]^{+}$: 453.1828; Found: 453.1829.

\section{Methyl Ether 46b}

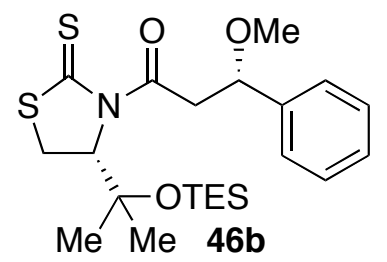

Clear yellow oil: ${ }^{1} \mathrm{H}$ NMR $\left(500 \mathrm{MHz}, \mathrm{CDCl}_{3}\right) \delta$ 7.38-7.33 (m, 4H), 7.33-7.28 (m, 1H), $5.30(\mathrm{~d}, J=8.1$, $1 \mathrm{H}), 4.82(\mathrm{dd}, J=9.5,3.3 \mathrm{~Hz}, 1 \mathrm{H}), 3.75(\mathrm{dd}, J=17.0,9.6 \mathrm{~Hz}, 1 \mathrm{H}), 3.49(\mathrm{dd}, J=16.8,3.6 \mathrm{~Hz}, 1 \mathrm{H}), 3.48$ $(\mathrm{dd}, J=8.1,3.3 \mathrm{~Hz}, 1 \mathrm{H}), 3.42(\mathrm{~d}, J=11.3 \mathrm{~Hz}, 1 \mathrm{H}), 3.21(\mathrm{~s}, 3 \mathrm{H}), 1.28(\mathrm{~s}, 3 \mathrm{H}), 1.24(\mathrm{~s}, 3 \mathrm{H}), 0.94(\mathrm{t}, J=$ $7.9 \mathrm{~Hz}, 6 \mathrm{H}), 0.60$ (q, $J=7.9 \mathrm{~Hz}, 4 \mathrm{H}) \mathrm{ppm} ;{ }^{13} \mathrm{C} \mathrm{NMR}\left(125 \mathrm{MHz}, \mathrm{CDCl}_{3}\right) \delta$ 204.5, 170.3, 140.5, 128.5, 128.0, 126.8, 79.6 76.7, 72.6, 56.6, 46.0, 30.3, 28.1, 26.2, 7.1, 6.6 ppm; IR (film) 2955, 2910, 2876, $2822,1736,1700,1456,1368,1312,1242,1148,1102,1036,971,934,745,724,701 \mathrm{~cm}^{-1} ;[\alpha]_{\mathrm{D}^{24}}=$ $-68.2^{\circ}\left(c=0.84, \mathrm{CHCl}_{3}\right)$; HRMS (EI): Exact mass calcd for $\mathrm{C}_{22} \mathrm{H}_{35} \mathrm{NO}_{3} \mathrm{~S}_{2} \mathrm{Si}[\mathrm{M}]^{+}:$453.1828; Found: 453.1855 .

\section{Methyl Ether 47a}

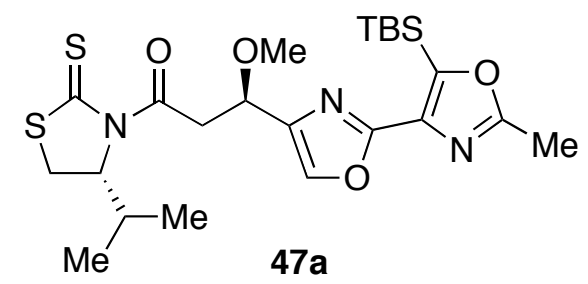

Clear yellow oil: ${ }^{1} \mathrm{H}$ NMR $\left(500 \mathrm{MHz}, \mathrm{CDCl}_{3}\right) \delta 7.67(\mathrm{~s}, 1 \mathrm{H}), 5.11(\mathrm{dd}, J=7.4,6.6 \mathrm{~Hz}, 1 \mathrm{H}), 4.84(\mathrm{dd}, J$ $=6.6,6.0 \mathrm{~Hz}, 1 \mathrm{H}), 4.02(\mathrm{dd}, J=17.8,7.1 \mathrm{~Hz}, 1 \mathrm{H}), 3.80(\mathrm{dd}, J=17.8,5.5 \mathrm{~Hz}, 1 \mathrm{H}), 3.49(\mathrm{dd}, J=11.4$, $8.1 \mathrm{~Hz}, 1 \mathrm{H}), 3.37(\mathrm{~s}, 3 \mathrm{H}), 3.02(\mathrm{~d}, J=11.4 \mathrm{~Hz}, 1 \mathrm{H}), 2.54(\mathrm{~s}, 3 \mathrm{H}), 2.42-2.34(\mathrm{~m}, 1 \mathrm{H}), 1.06(\mathrm{~d}, J=6.7 \mathrm{~Hz}$, $3 \mathrm{H}), 0.98(\mathrm{~d}, J=6.9 \mathrm{~Hz}, 3 \mathrm{H}), 0.95(\mathrm{~s}, 9 \mathrm{H}), 0.39$ (s, 3H), $0.38(\mathrm{~s}, 3 \mathrm{H}) \mathrm{ppm} ;{ }^{13} \mathrm{C} \mathrm{NMR}\left(125 \mathrm{MHz}, \mathrm{CDCl}_{3}\right)$ o 202.3, 170.9, 164.9, 156.5, 154.7, 140.8, 138.8, 135.4, 72.2, 71.4, 56.8, 43.5, 30.6, 30.1, 26.3, 18.9, $17.5,17.4,13.7,-5.99,-6.01$ ppm; IR (film) 2957, 2930, 2895, 2857, 1698, 1613, 1585, 1469, 1364, 1314, 1252, 1163, 1103, 1039, 930, 842, $782 \mathrm{~cm}^{-1} ;[\alpha]_{\mathrm{D}}^{24}=-238.7^{\circ}\left(c=1.00, \mathrm{CHCl}_{3}\right)$; HRMS (EI): Exact mass calcd for $\mathrm{C}_{23} \mathrm{H}_{35} \mathrm{~N}_{3} \mathrm{O}_{4} \mathrm{~S}_{2} \mathrm{Si}[\mathrm{M}]^{+}$: 509.1838; Found: 509.1840.

\section{Methyl Ether 48a}

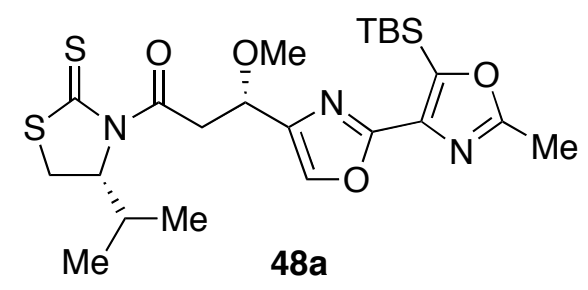


Clear yellow oil: ${ }^{1} \mathrm{H}$ NMR $\left(500 \mathrm{MHz}, \mathrm{CDCl}_{3}\right) \delta 7.65(\mathrm{~s}, 1 \mathrm{H}), 5.17(\mathrm{dd}, J=6.9,6.9 \mathrm{~Hz}, 1 \mathrm{H}), 4.89$ (dd, $J$ $=9.4,2.9 \mathrm{~Hz}, 1 \mathrm{H}), 3.87(\mathrm{dd}, J=17.9,9.5 \mathrm{~Hz}, 1 \mathrm{H}), 3.65(\mathrm{dd}, J=17.9,2.9 \mathrm{~Hz}, 1 \mathrm{H}), 3.56(\mathrm{dd}, J=11.4$, $7.9 \mathrm{~Hz}, 1 \mathrm{H}), 3.38(\mathrm{~s}, 3 \mathrm{H}), 3.04(\mathrm{~d}, J=11.7 \mathrm{~Hz}, 1 \mathrm{H}), 2.54(\mathrm{~s}, 3 \mathrm{H}), 2.42-2.35(\mathrm{~m}, 1 \mathrm{H}), 1.07$ (d, $J=6.9 \mathrm{~Hz}$, $3 \mathrm{H}), 0.98(\mathrm{~d}, J=7.1 \mathrm{~Hz}, 3 \mathrm{H}), 0.96(\mathrm{~s}, 9 \mathrm{H}), 0.40(\mathrm{~s}, 3 \mathrm{H}), 0.39$ (s, 3H) ppm; ${ }^{13} \mathrm{C}$ NMR $\left(125 \mathrm{MHz}, \mathrm{CDCl}_{3}\right)$

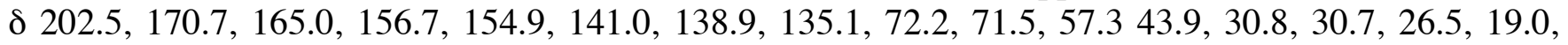
17.8, 17.6, 13.8, -5.8 ppm; IR (film) 2956, 2929, 2857, 1698, 1466, 1364, 1315, 1250, 1165, 1104, 1040, 930, 841, 823, 811, 781, $680 \mathrm{~cm}^{-1} ;[\alpha]_{\mathrm{D}^{24}}=-111.3^{\circ}\left(c=1.02, \mathrm{CHCl}_{3}\right)$; HRMS (EI): Exact mass calcd for $\mathrm{C}_{23} \mathrm{H}_{35} \mathrm{~N}_{3} \mathrm{O}_{4} \mathrm{~S}_{2} \mathrm{Si}[\mathrm{M}]+:$ : 509.1838; Found: 509.1847 . 


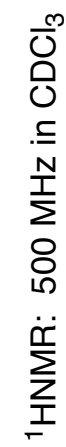

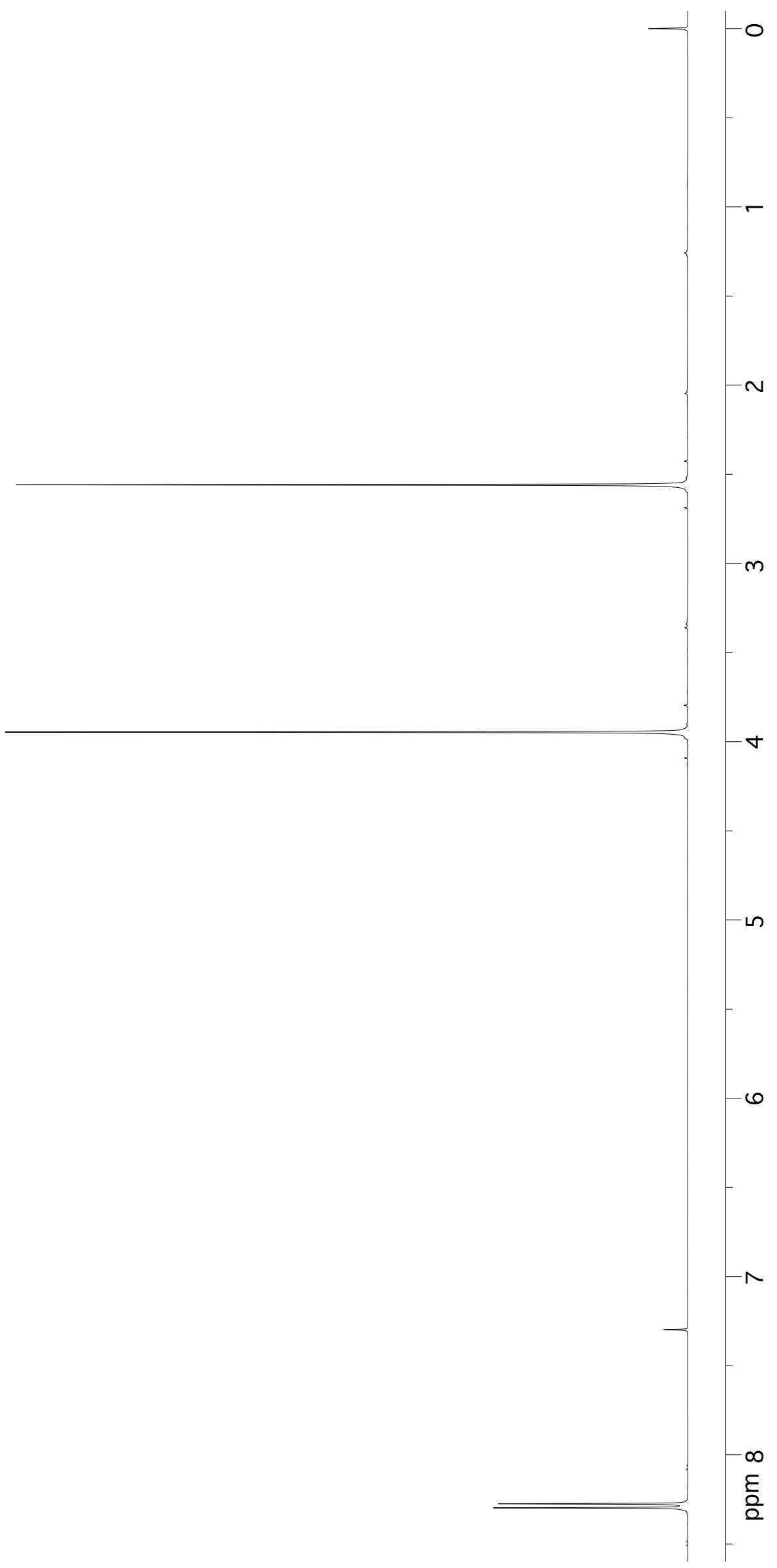




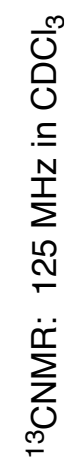

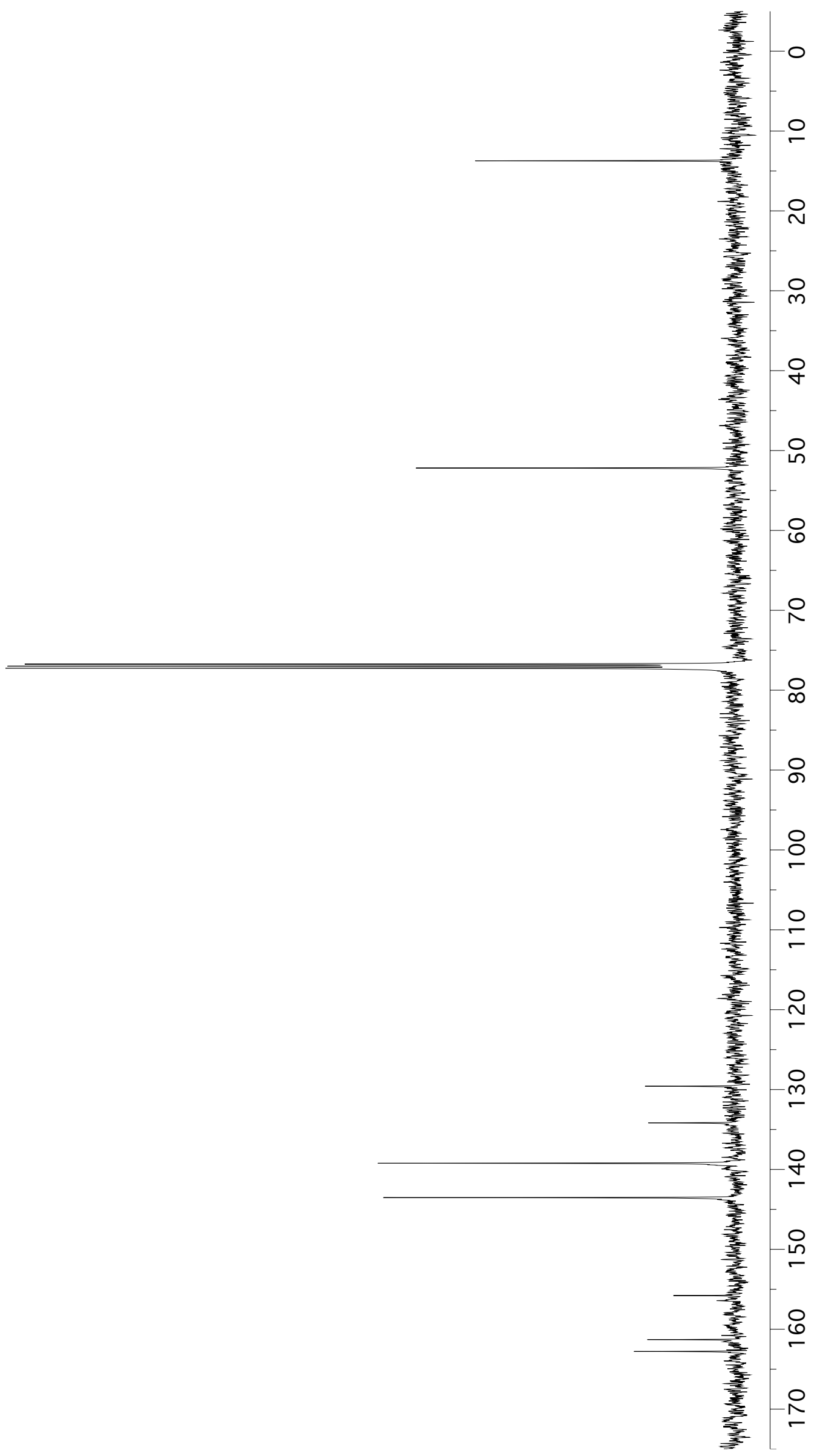




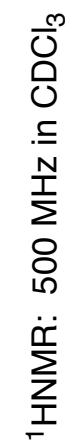

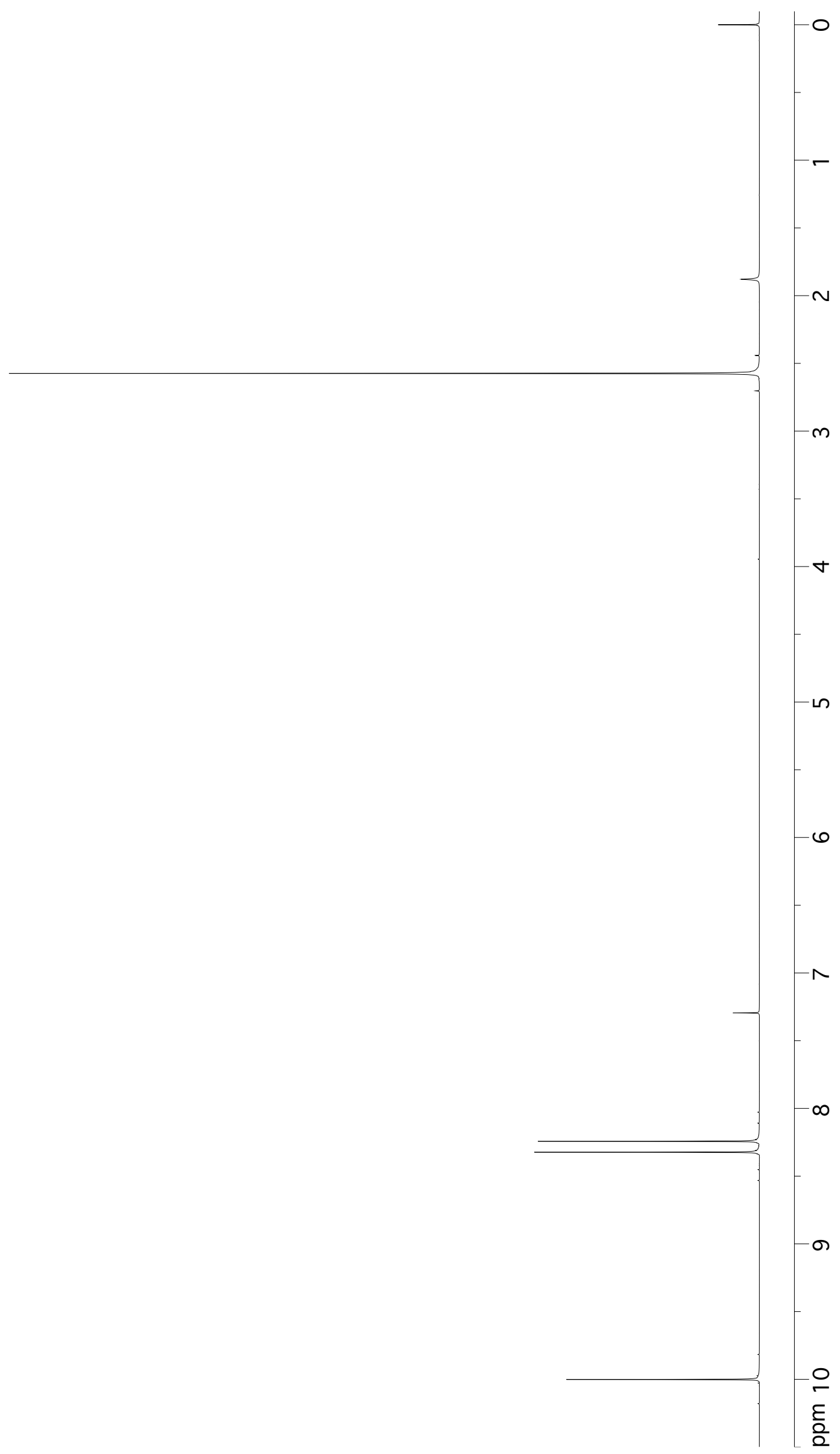




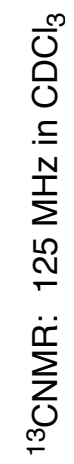

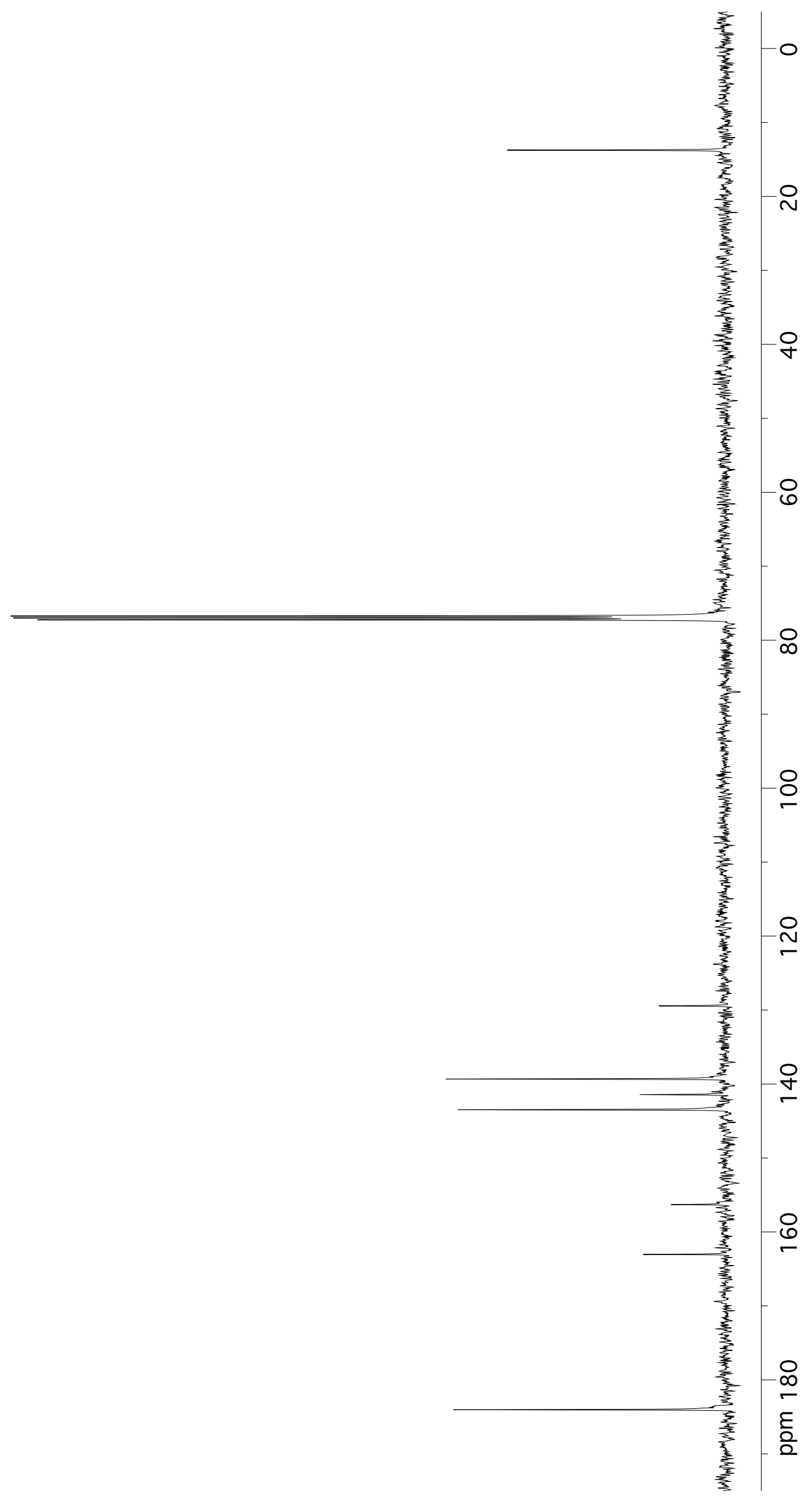




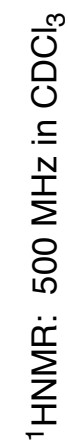

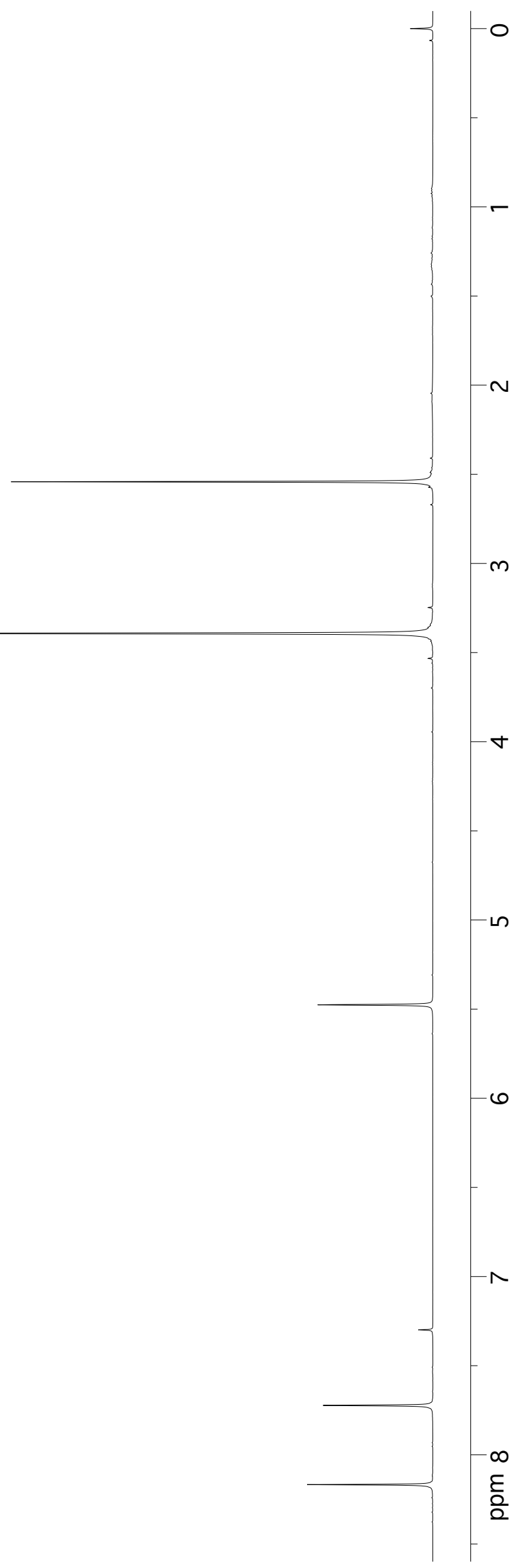




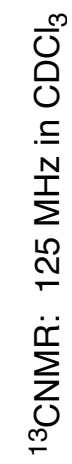

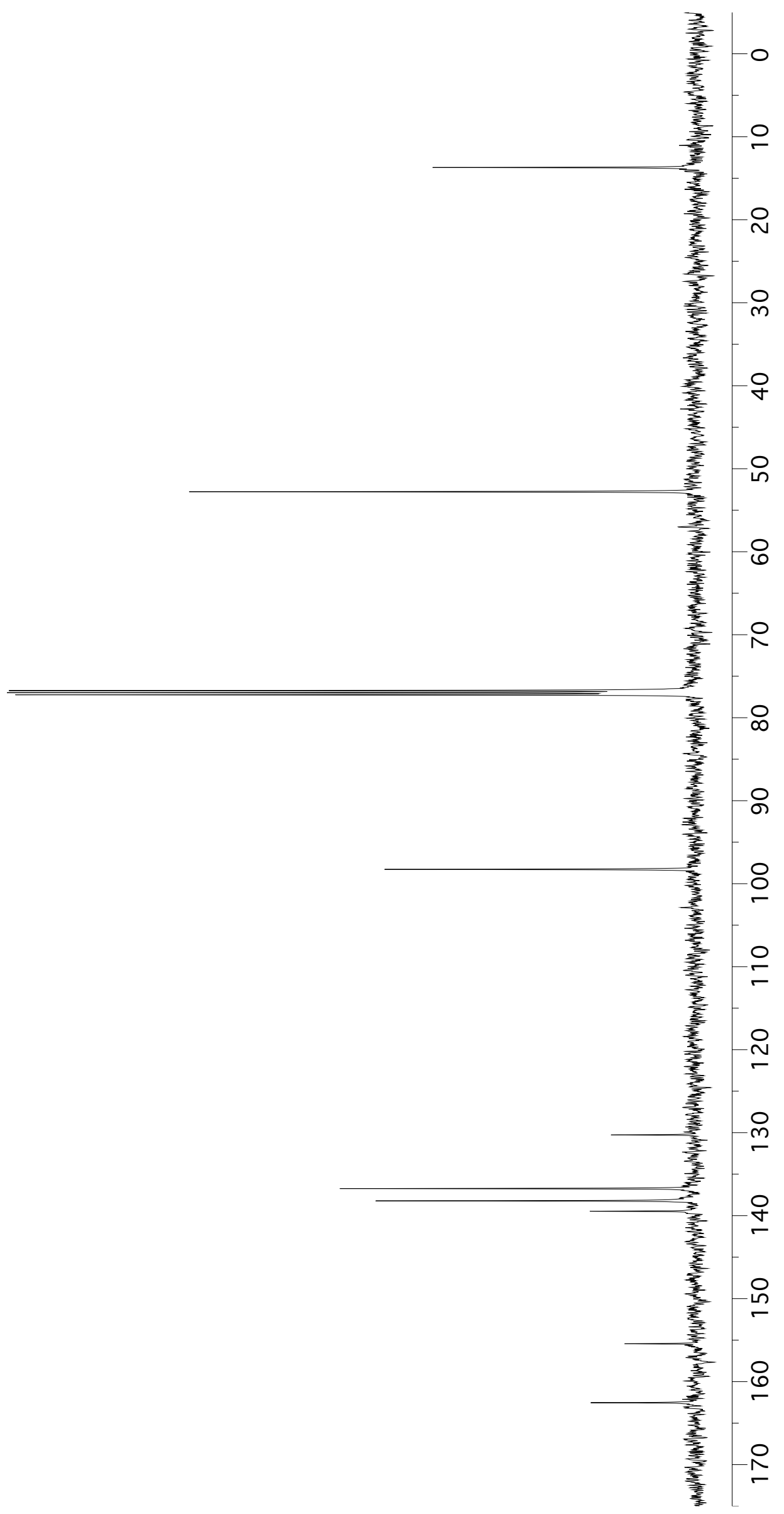




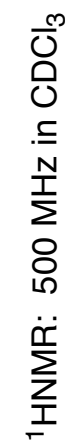

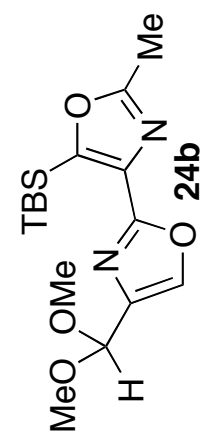

$\rightarrow+0$ 


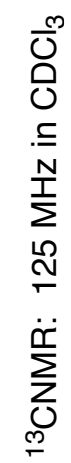

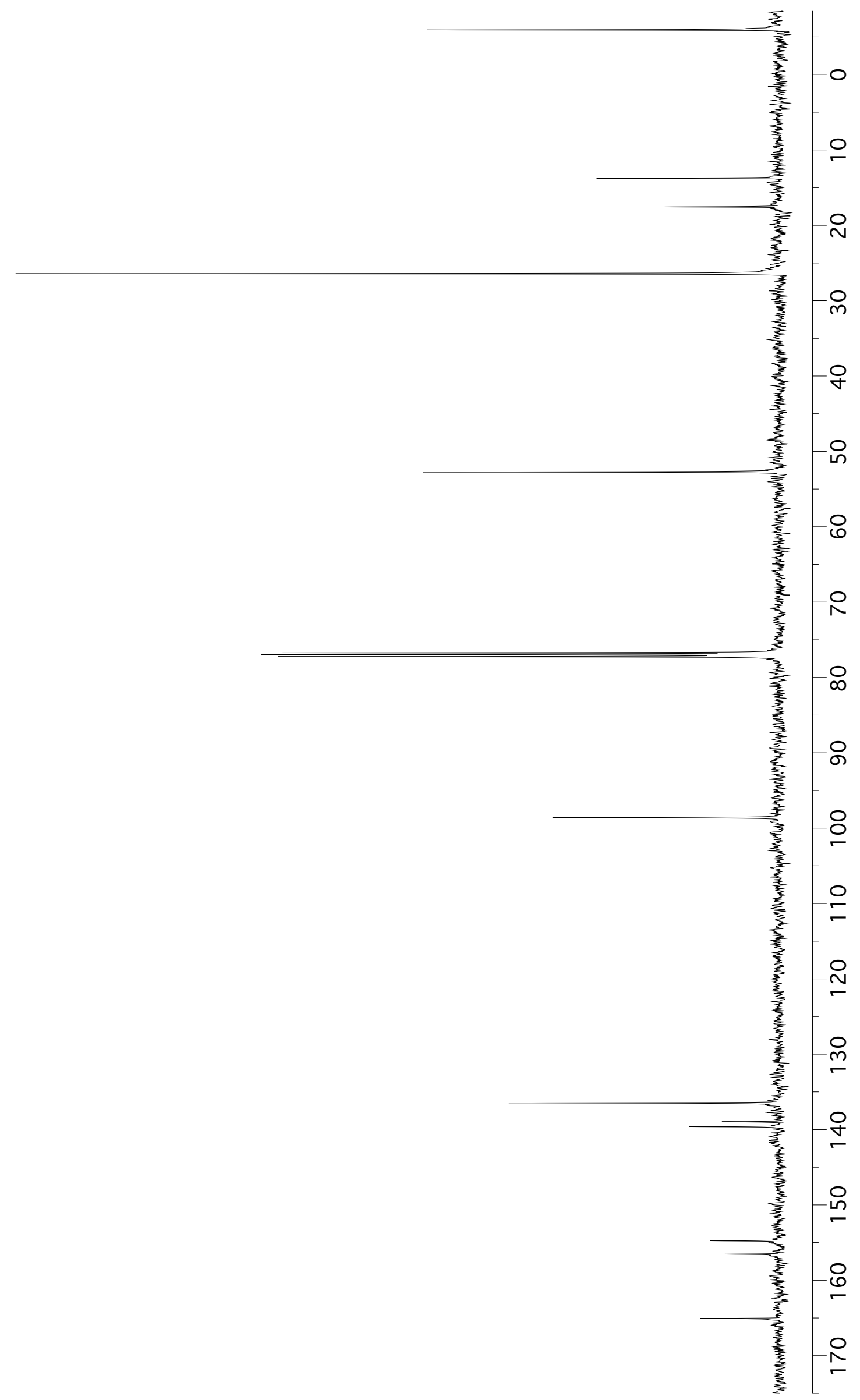




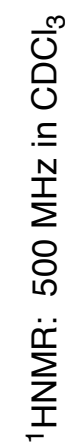
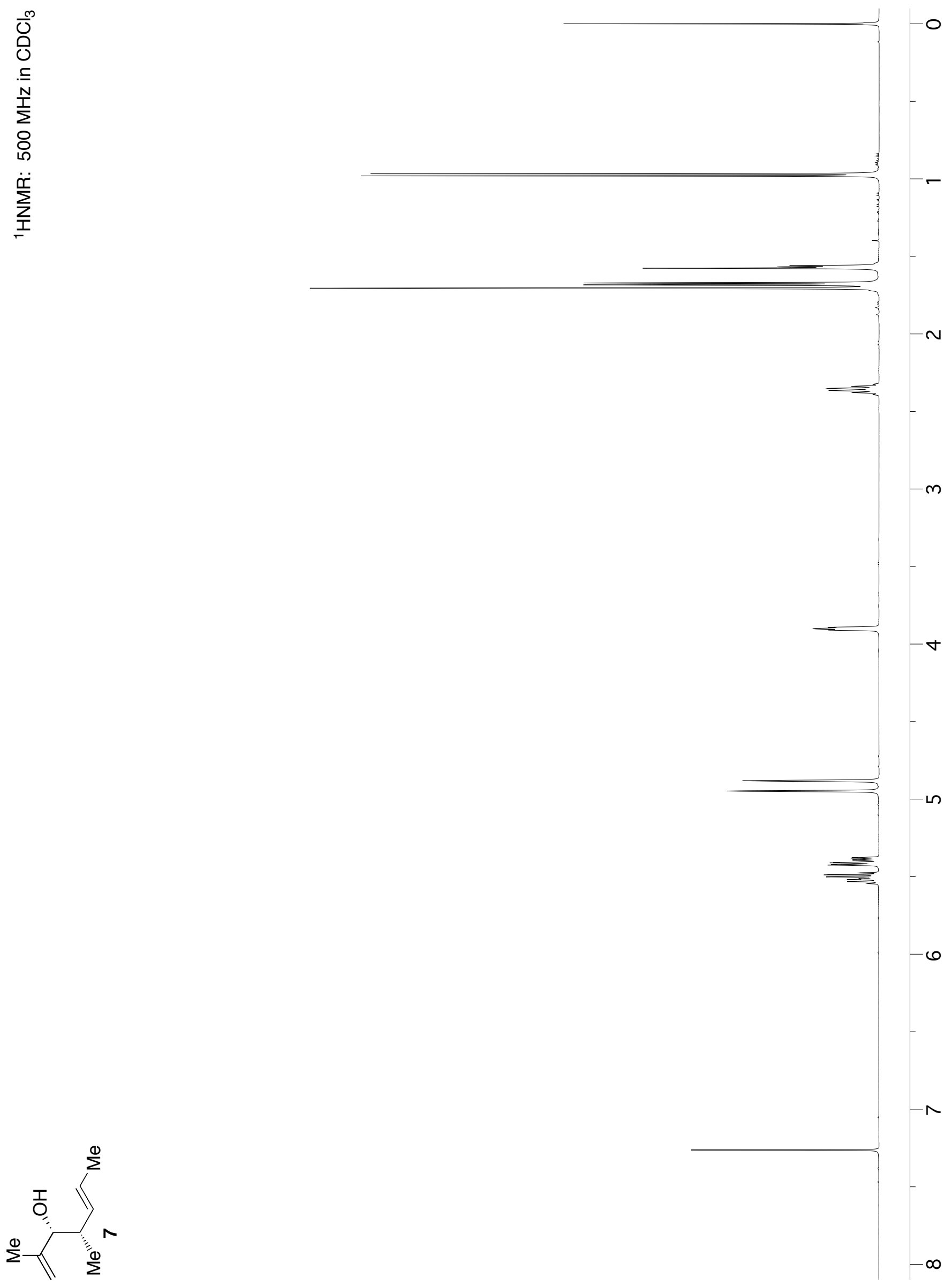


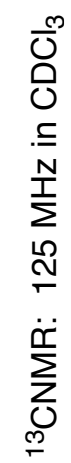

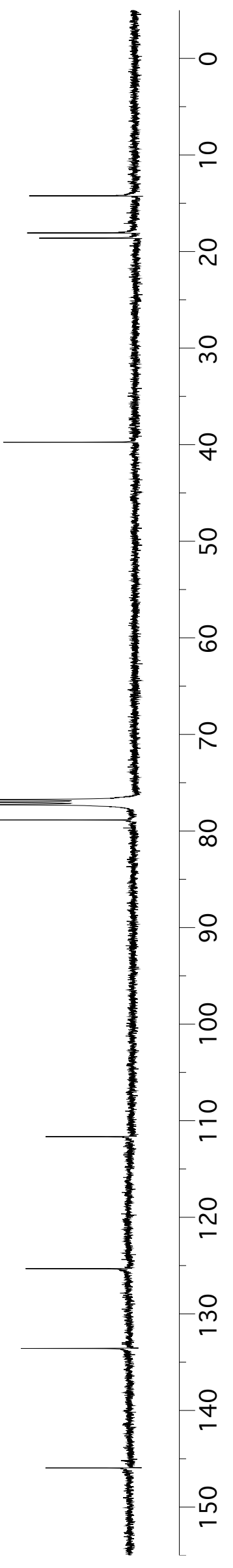




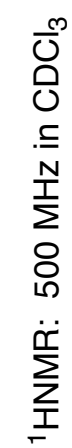

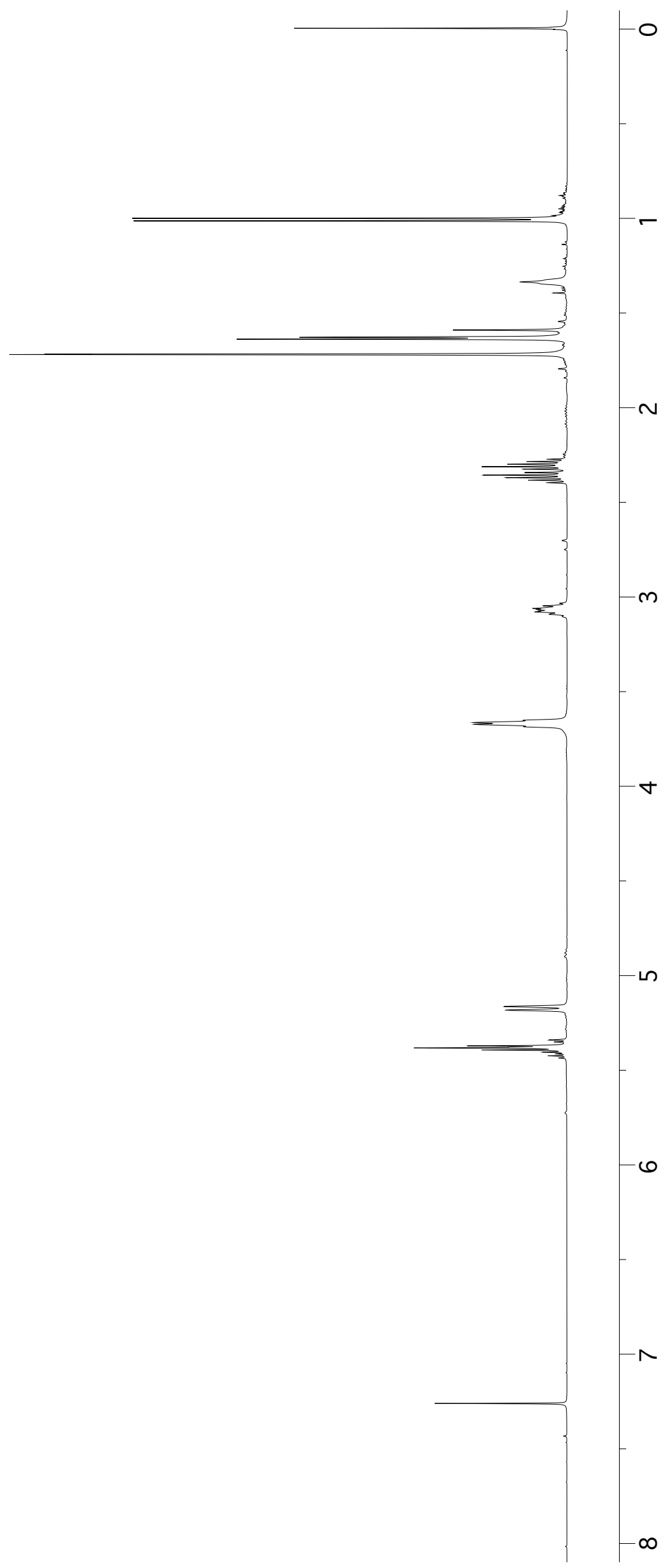




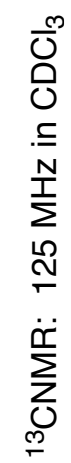

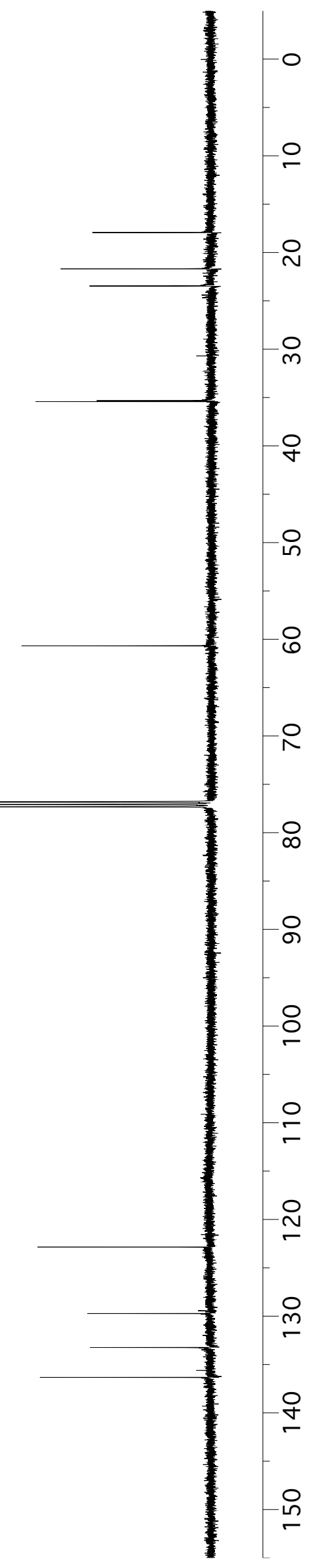




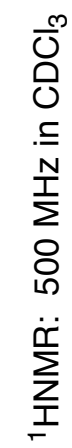

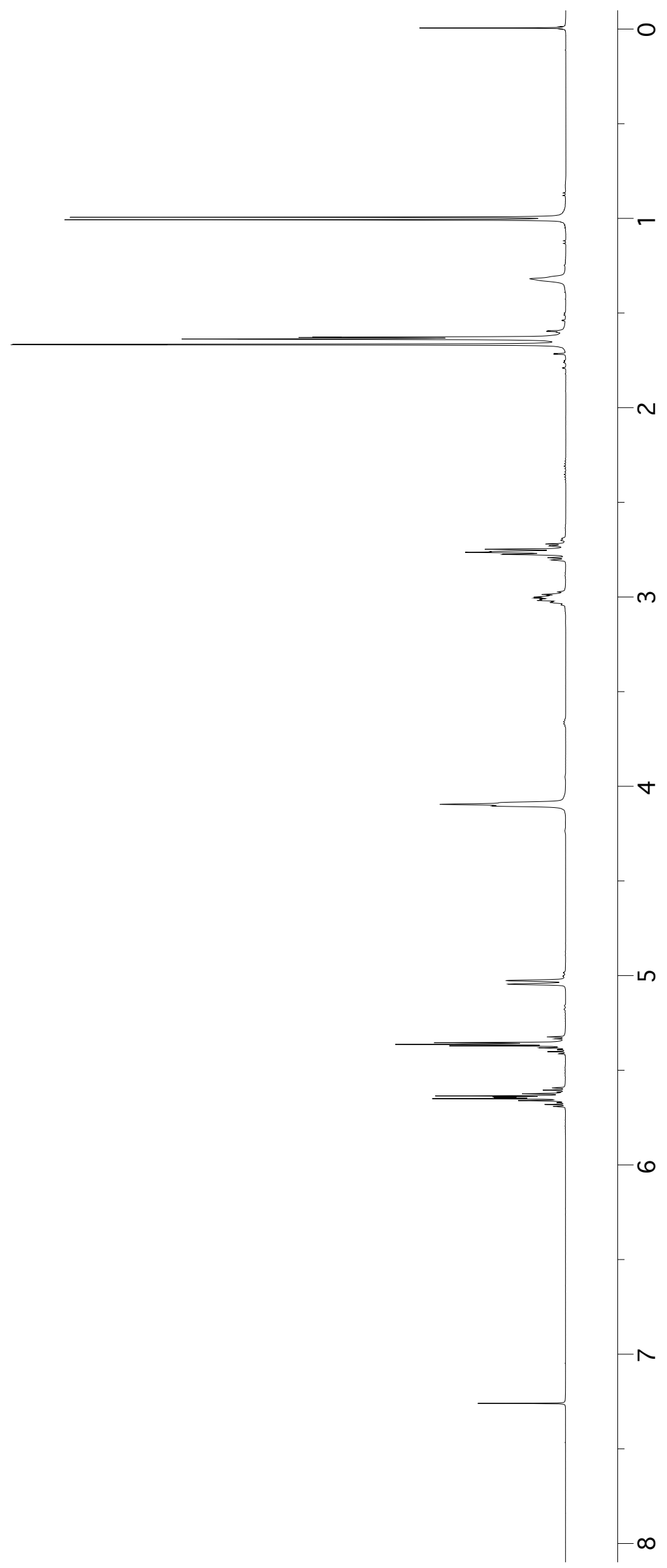




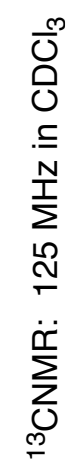

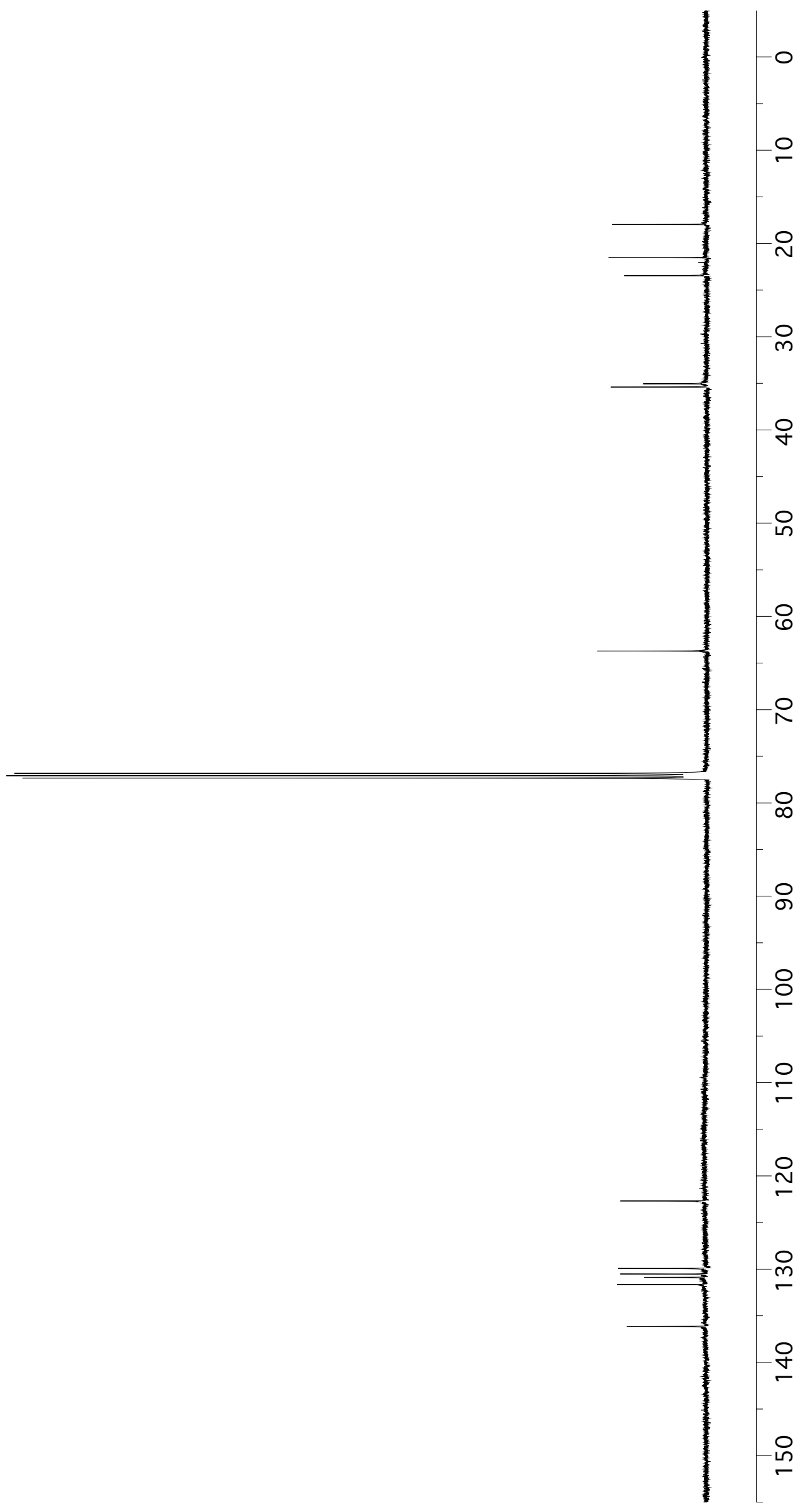




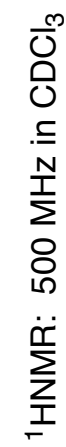

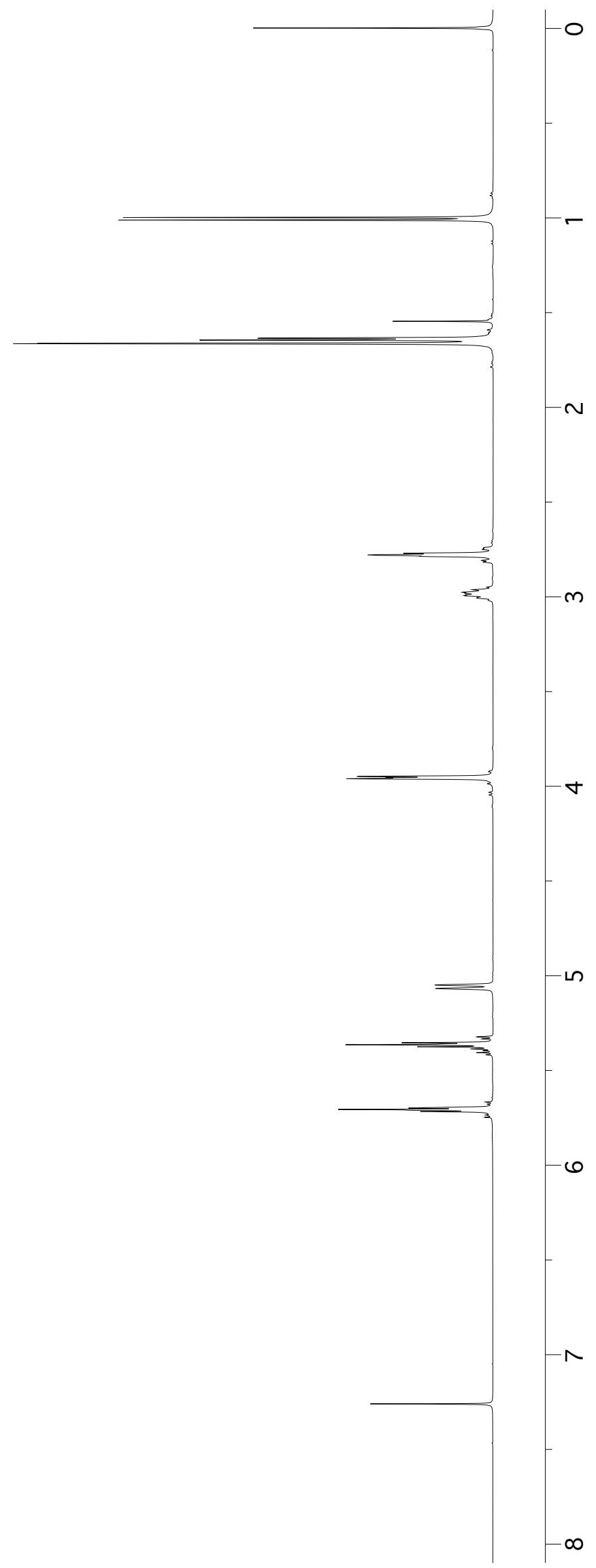




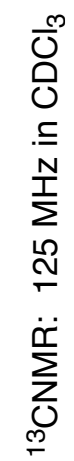

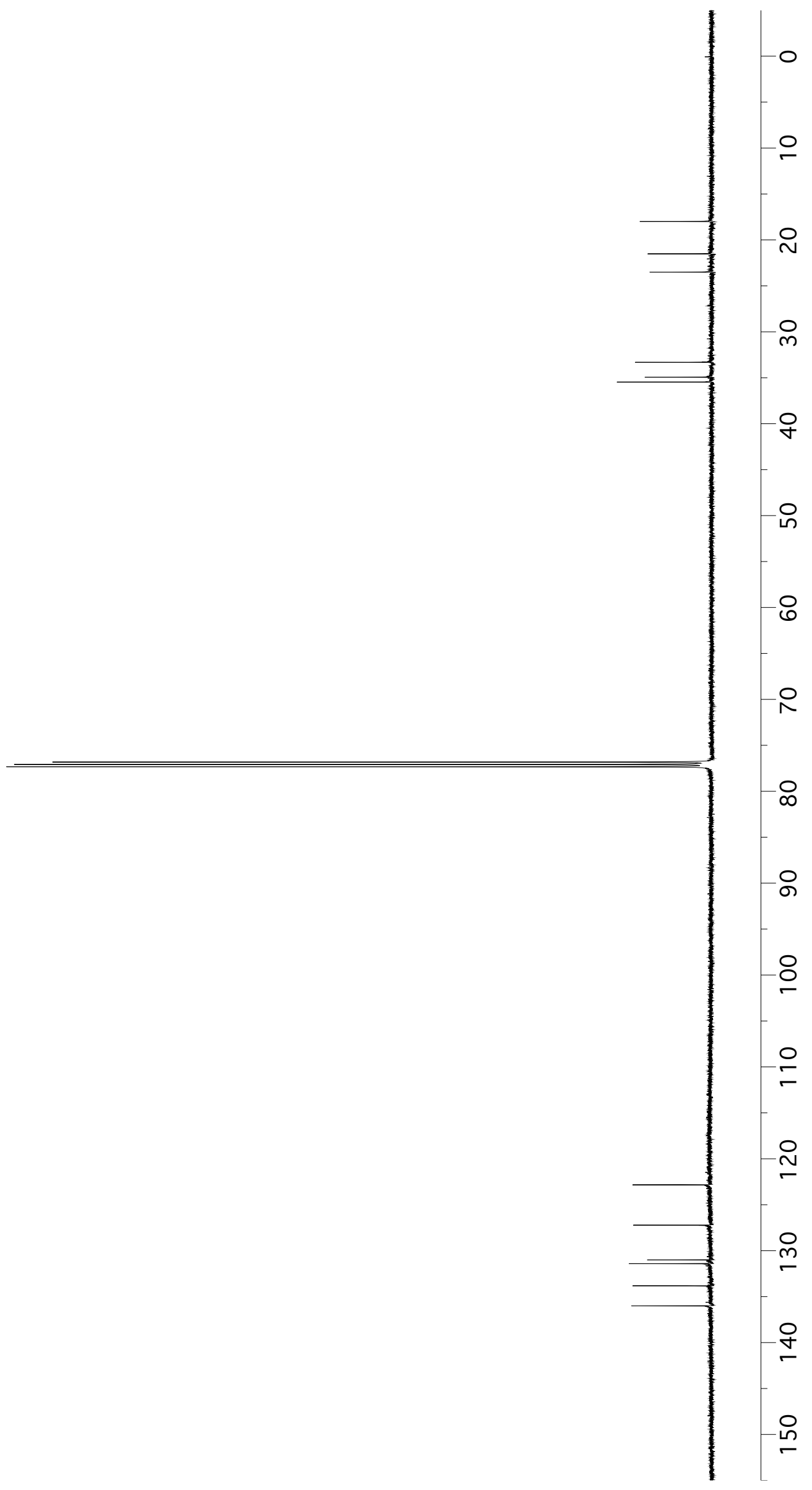




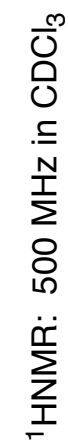

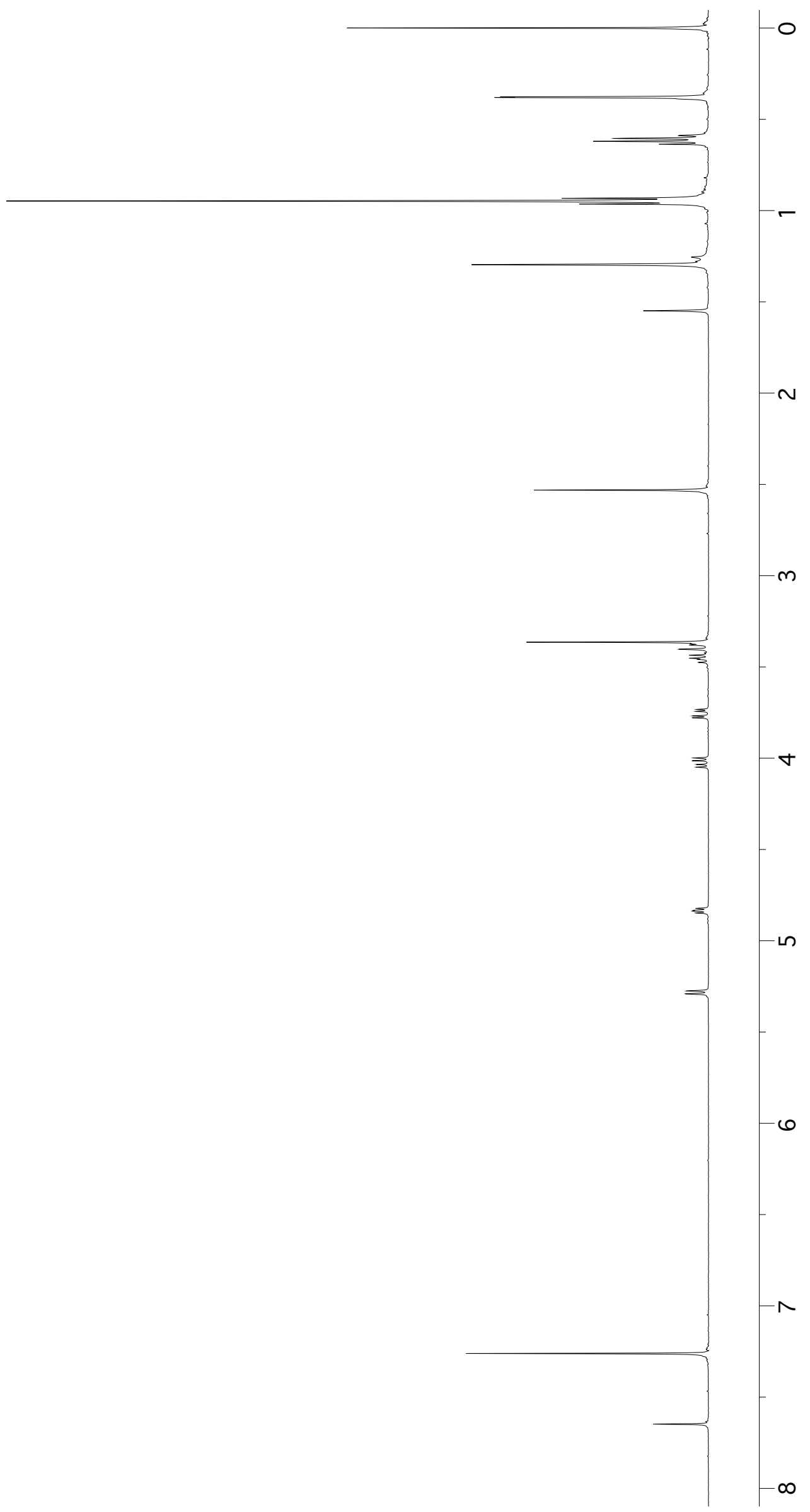




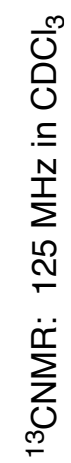
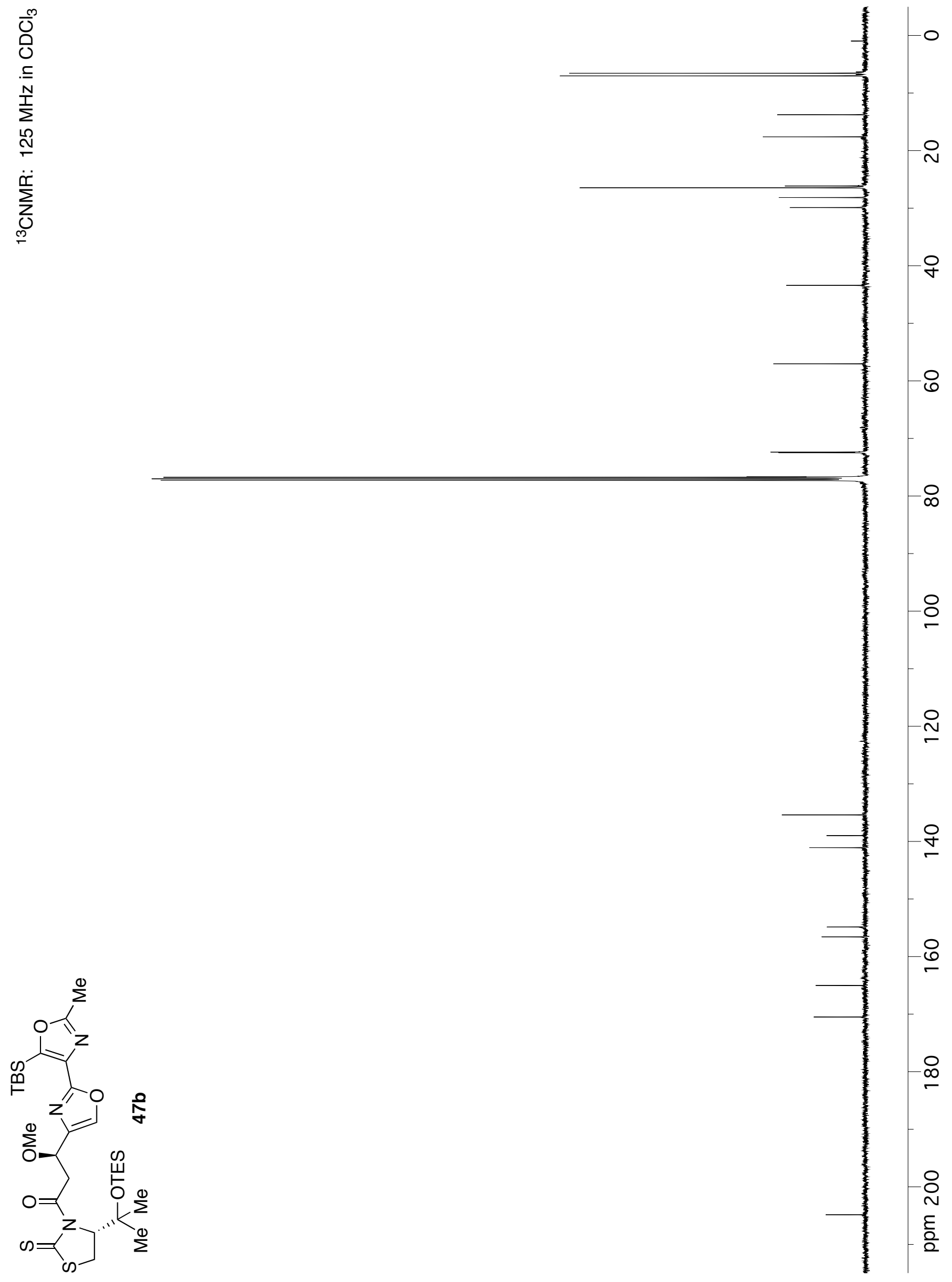


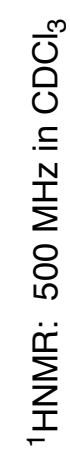
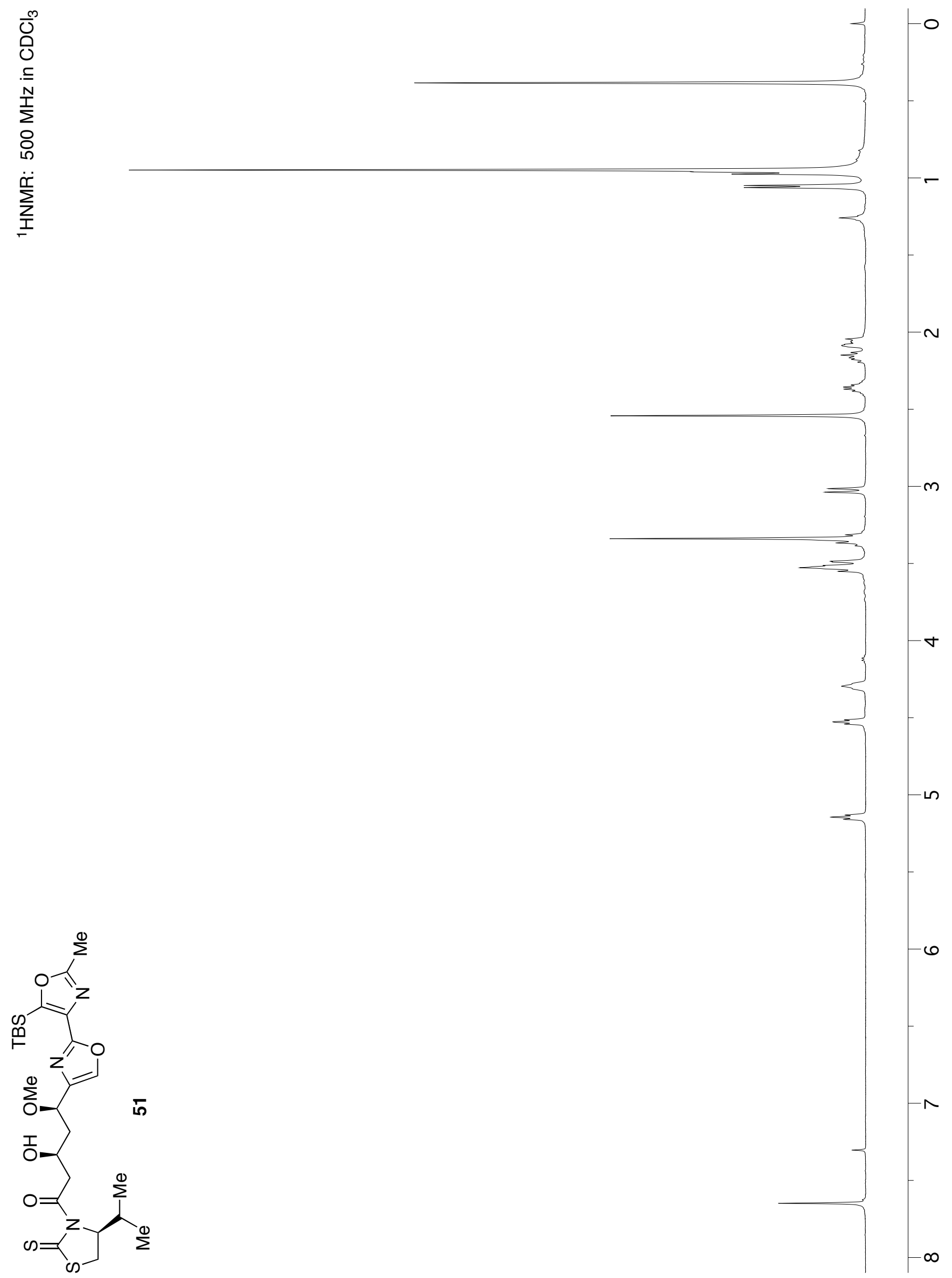


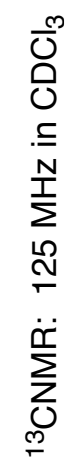

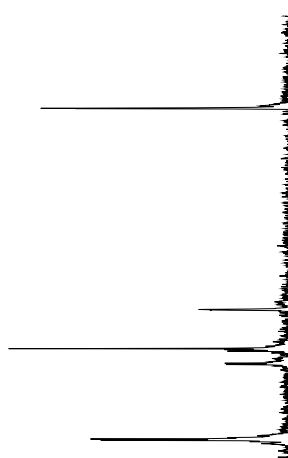

-
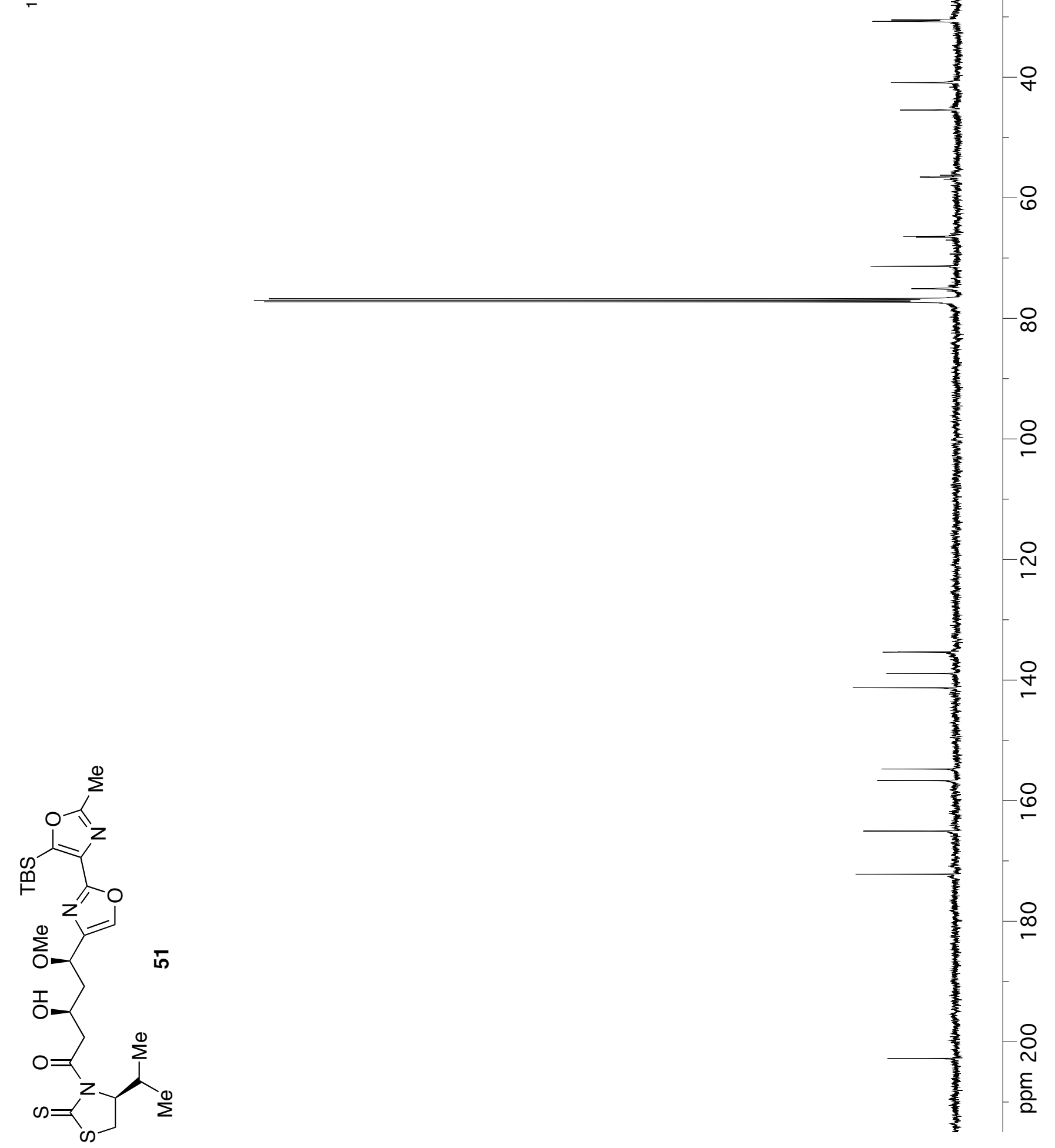


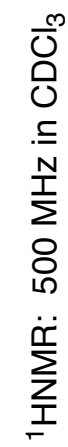

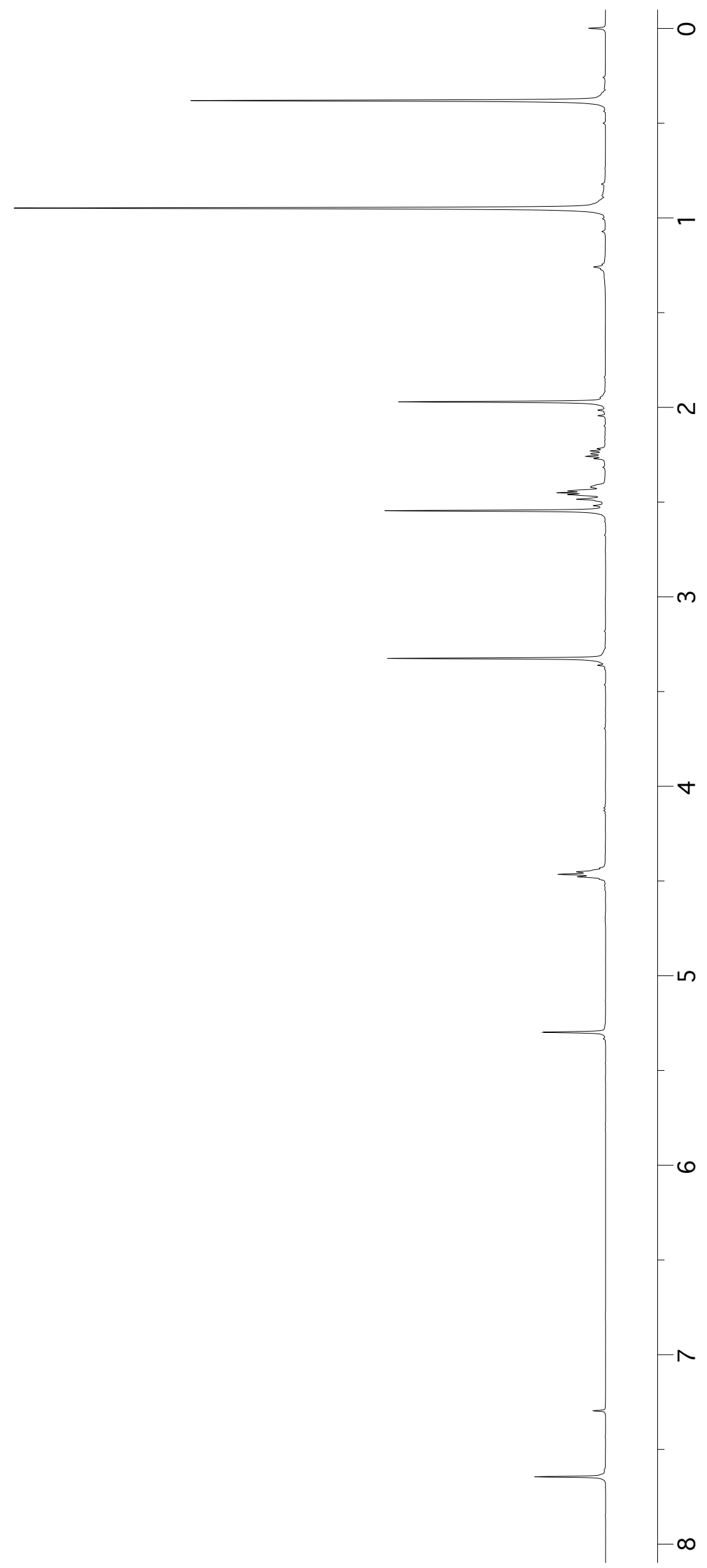




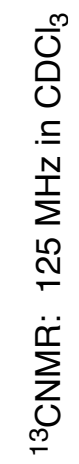
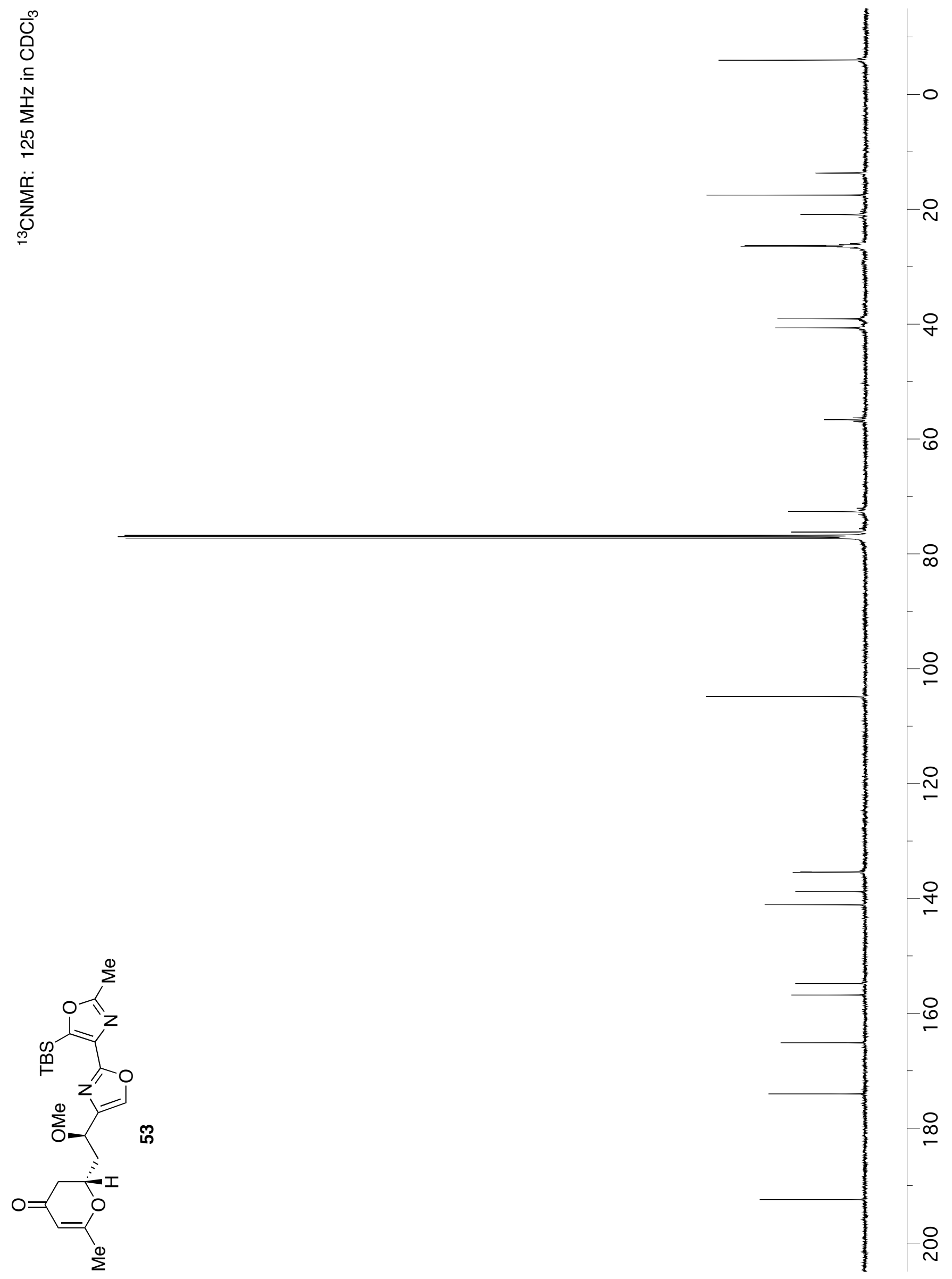


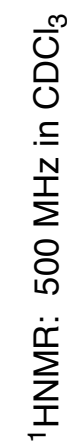

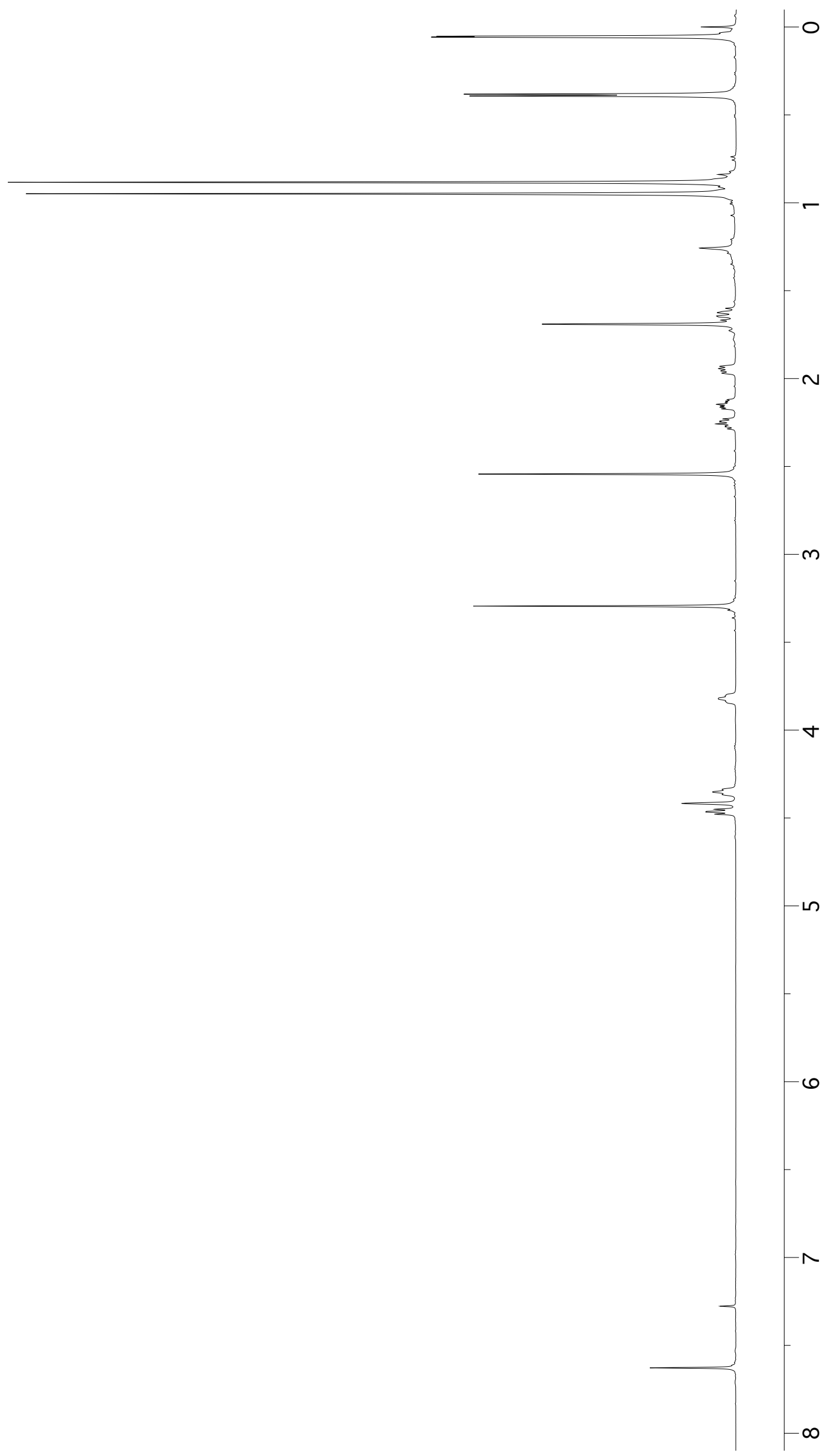




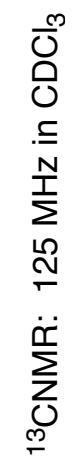
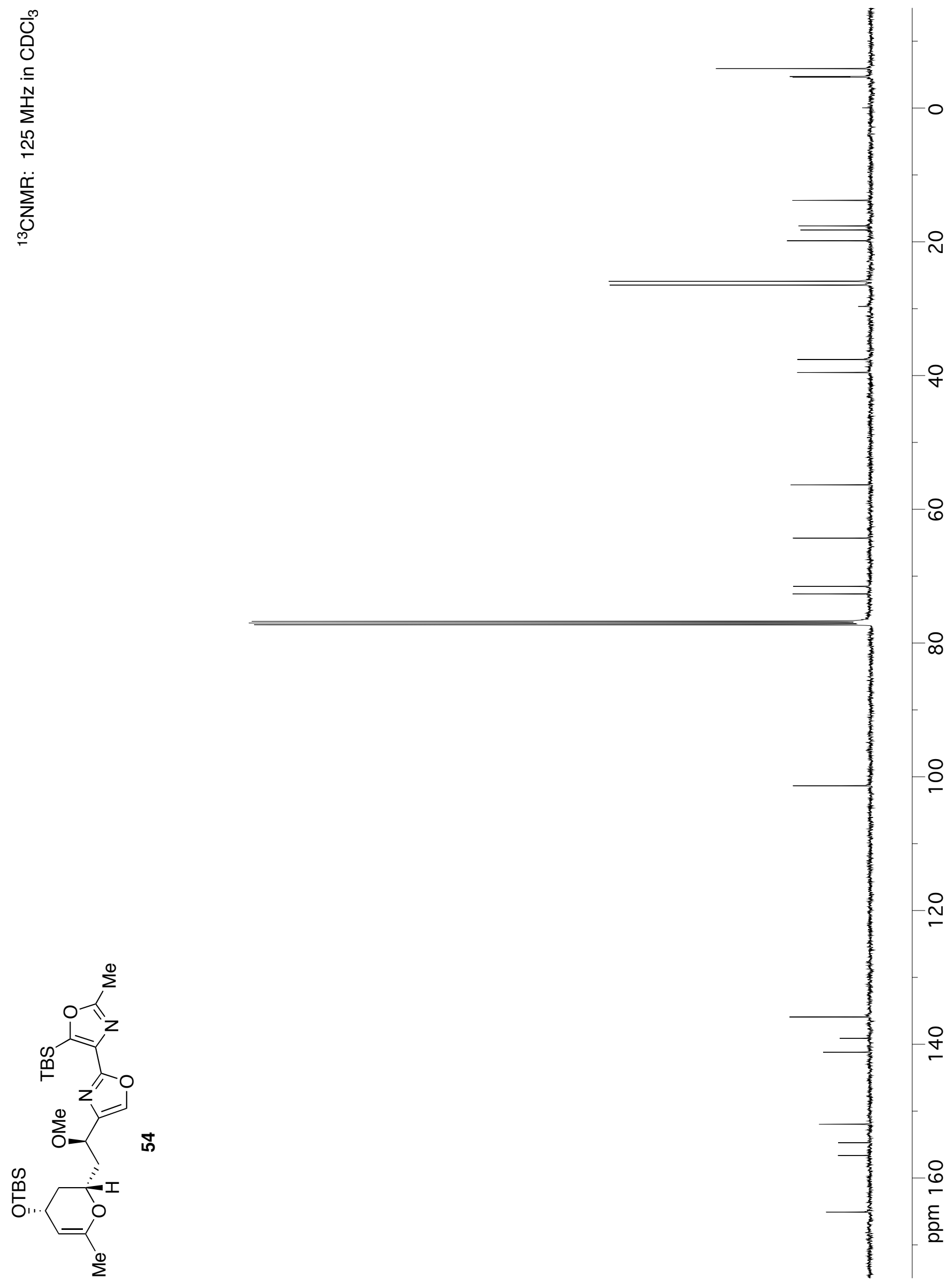


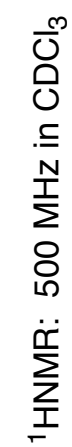

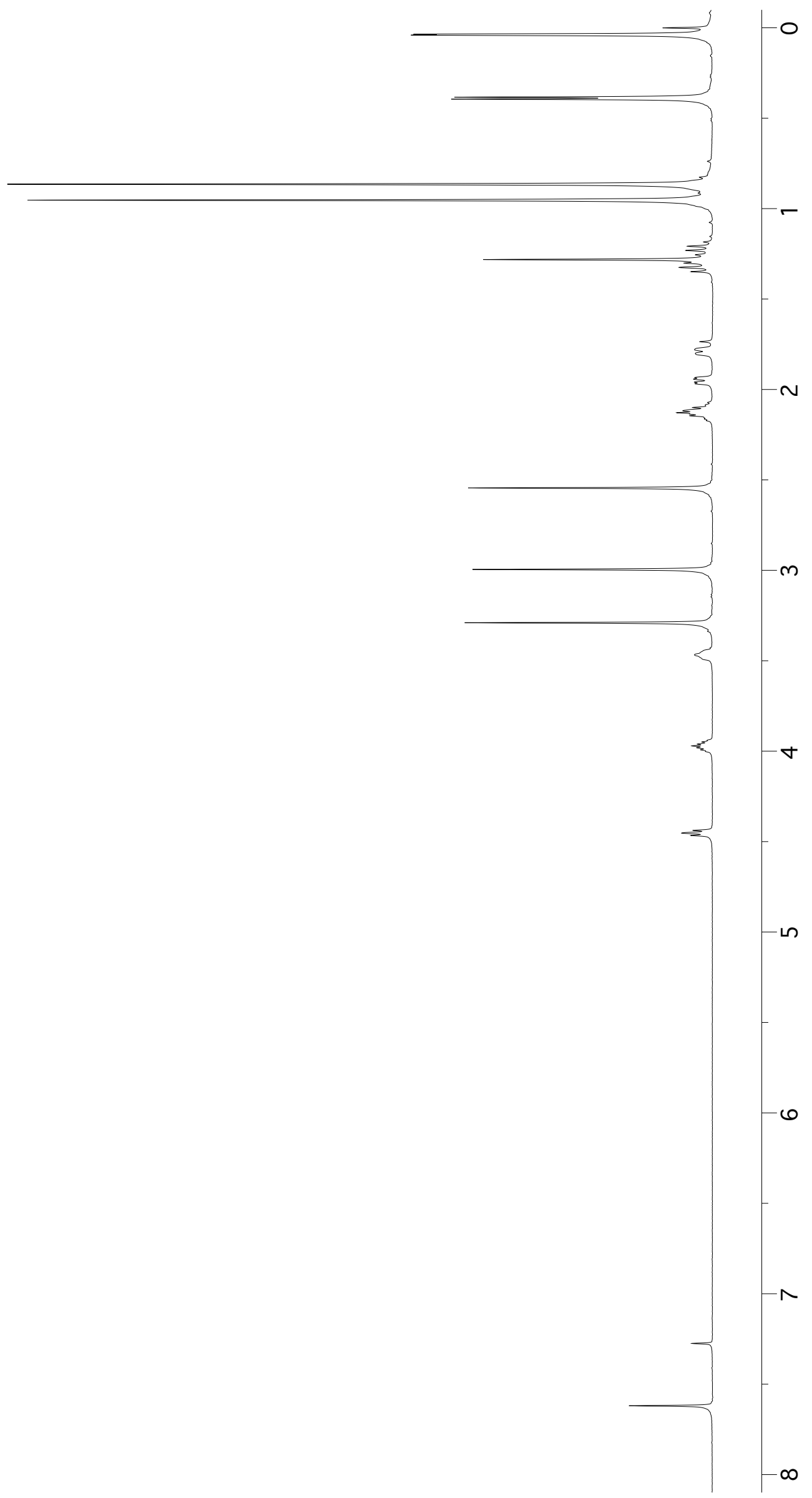




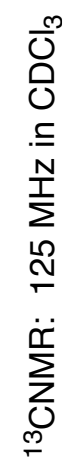

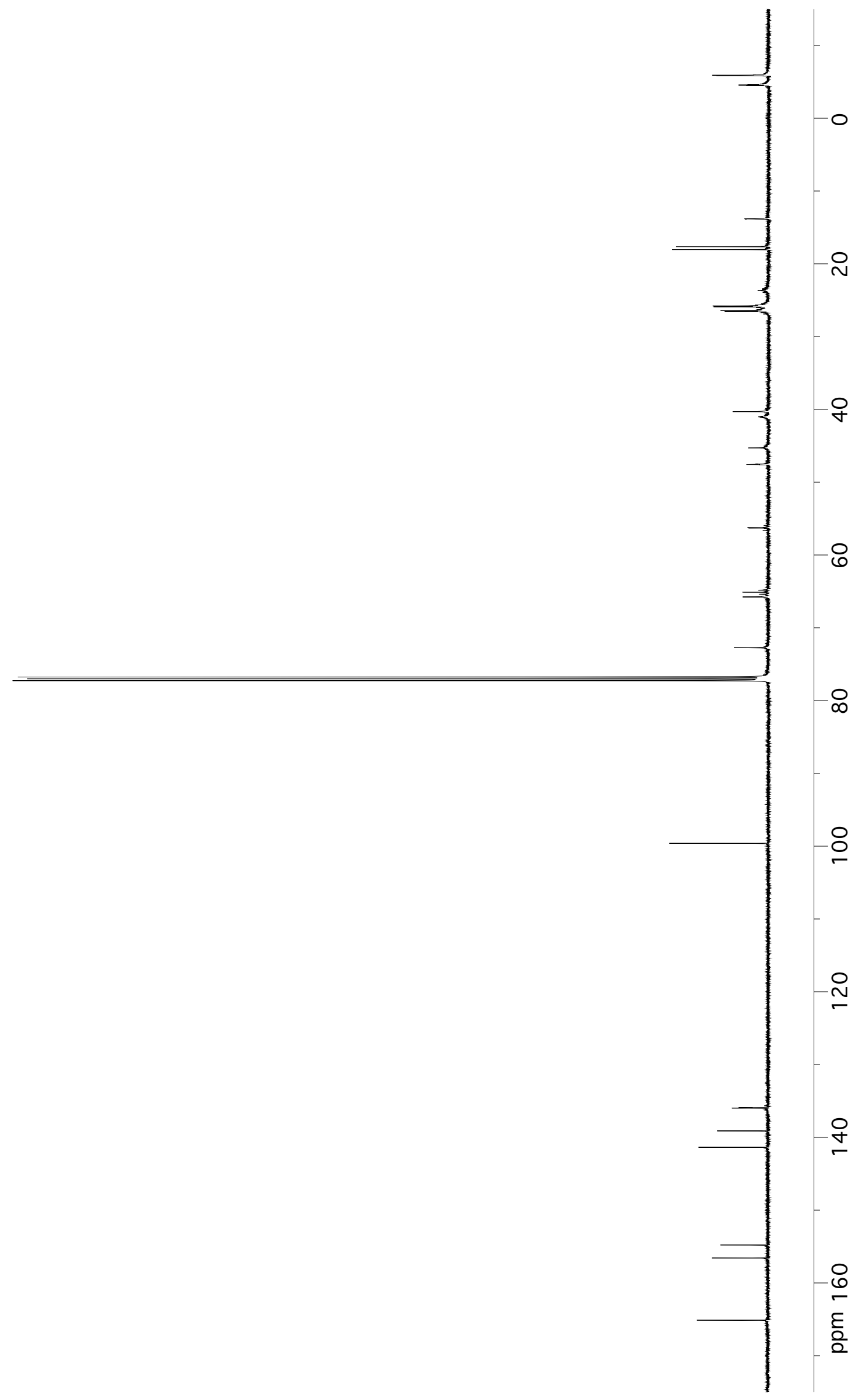




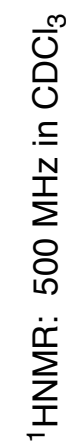

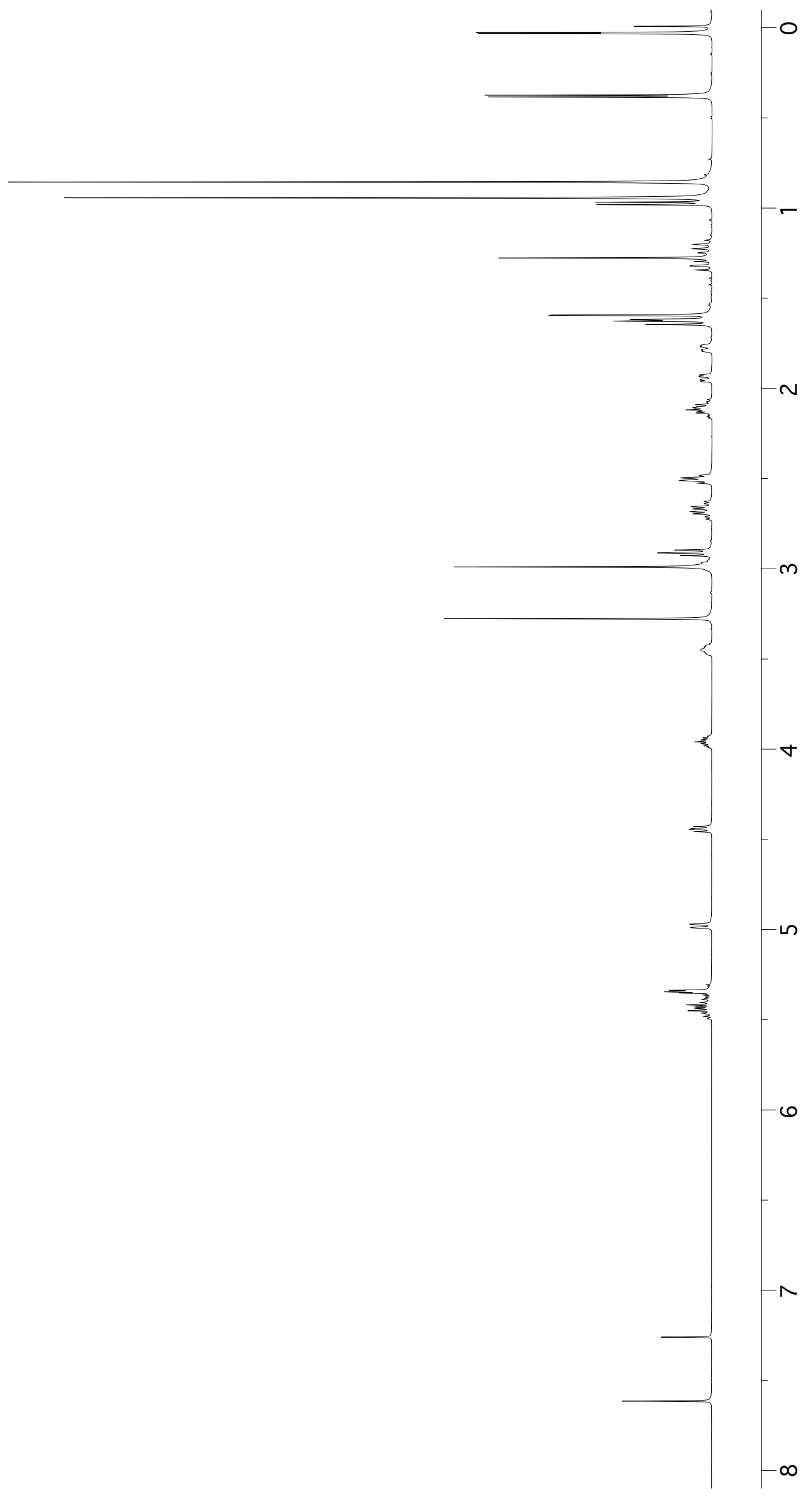




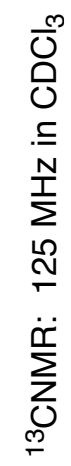

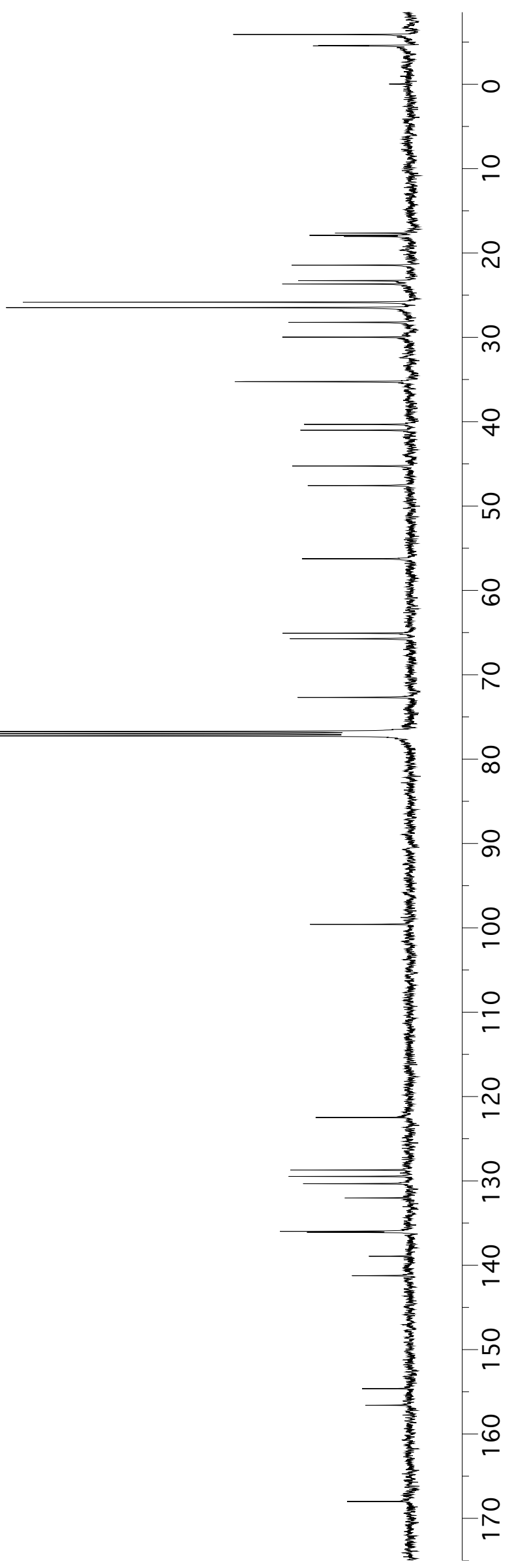




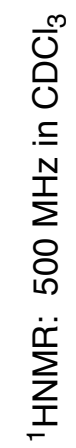

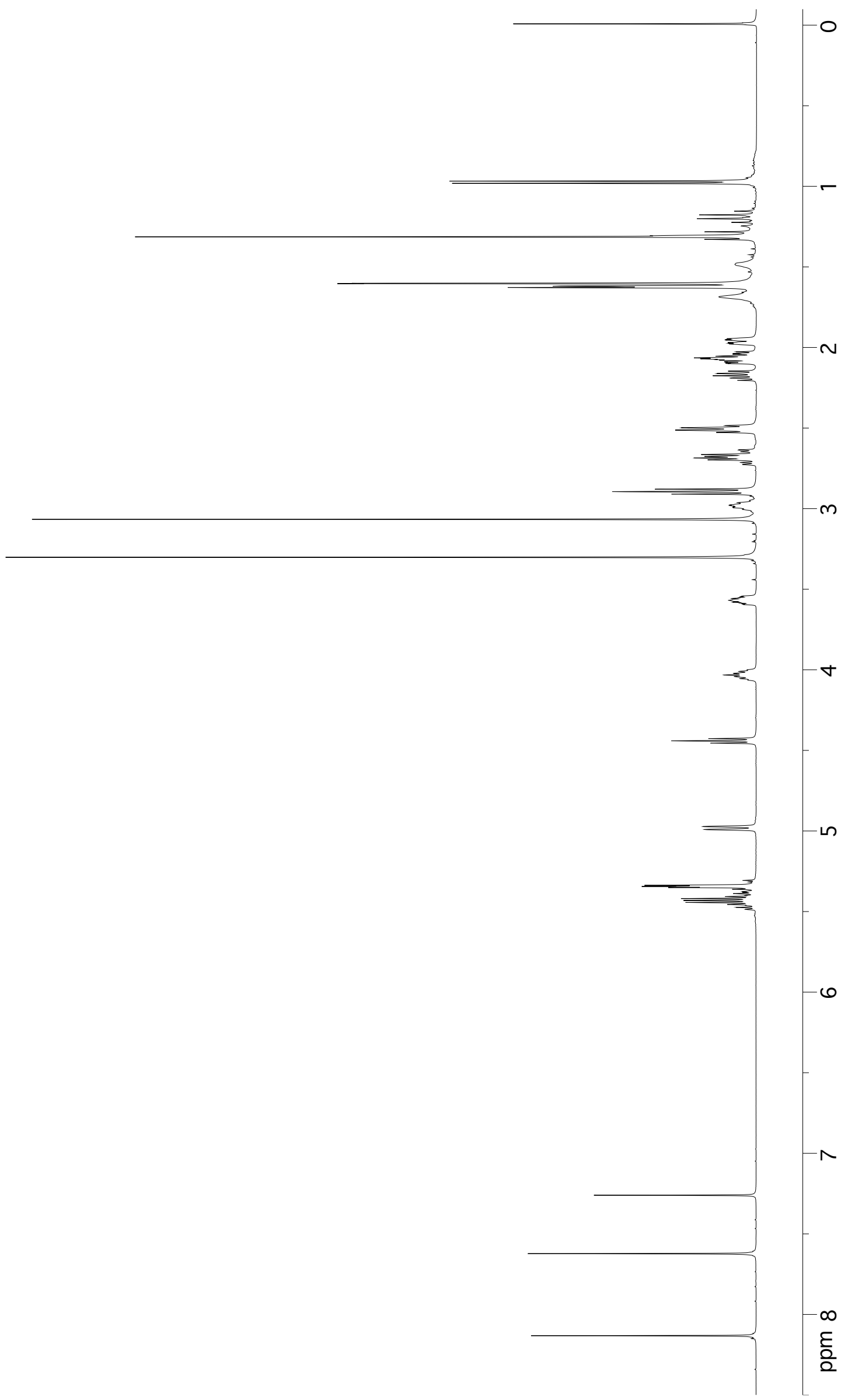




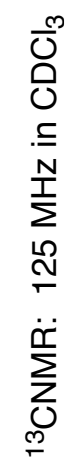
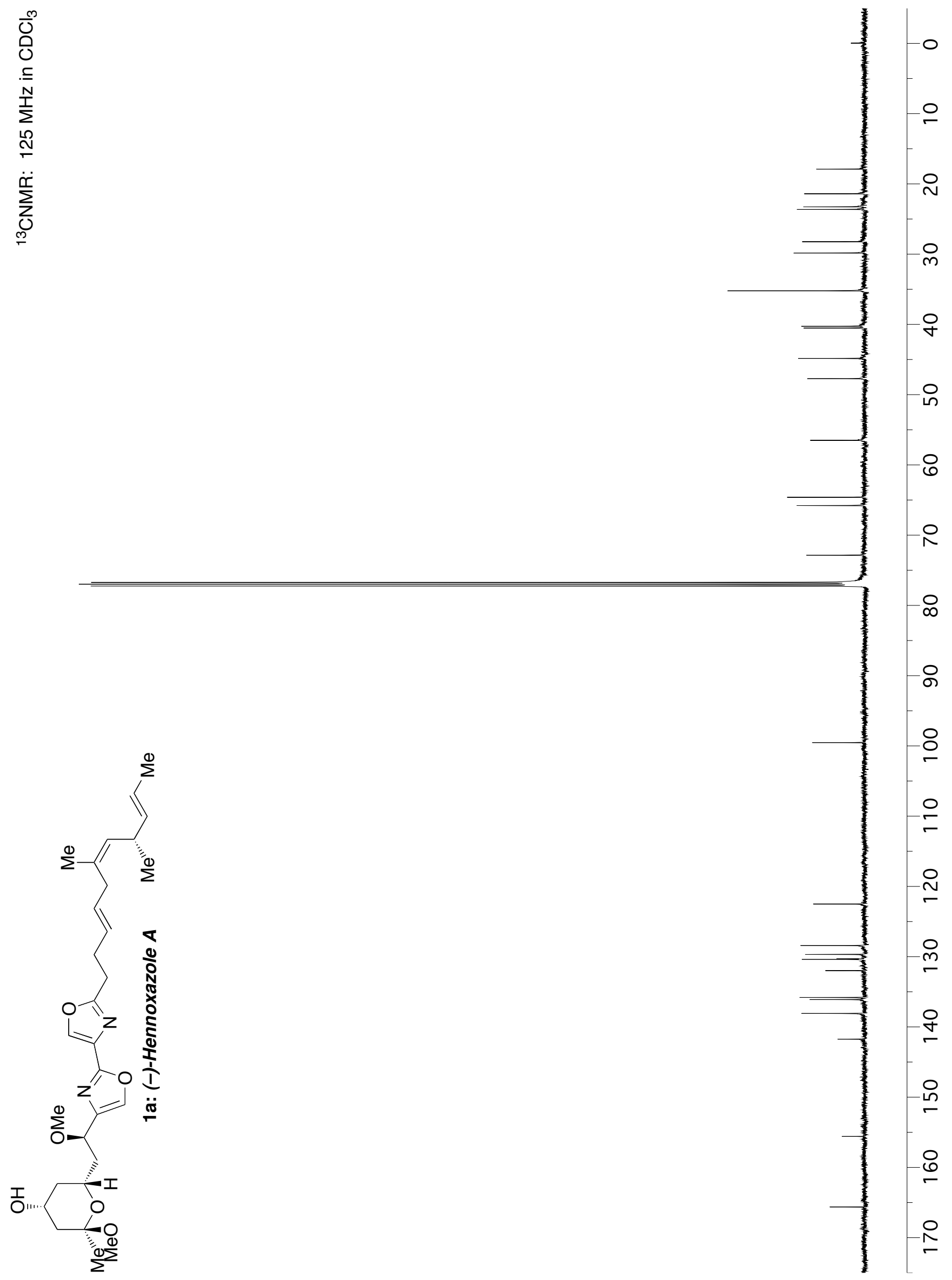


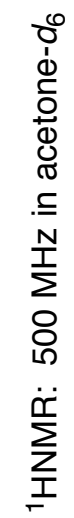

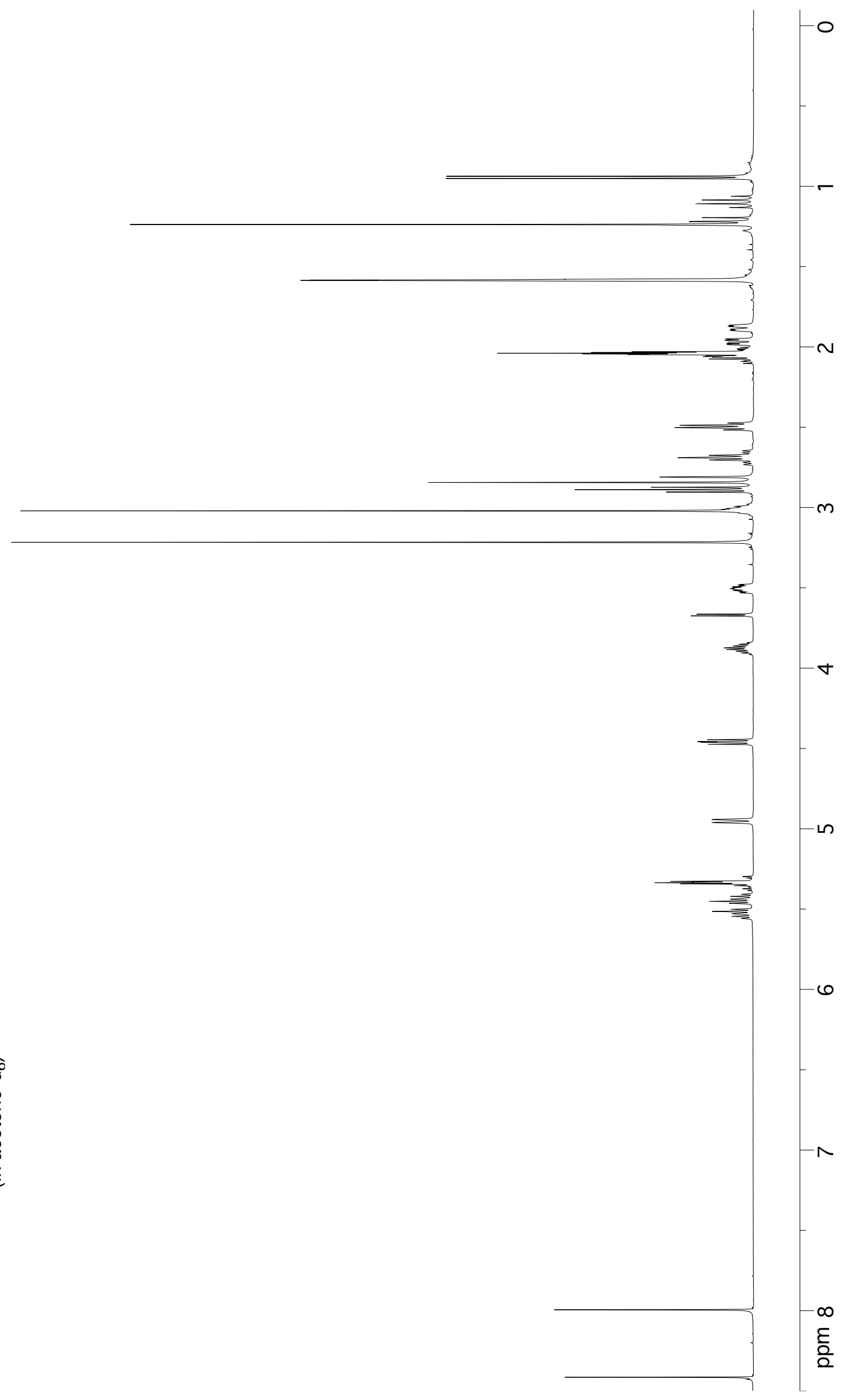




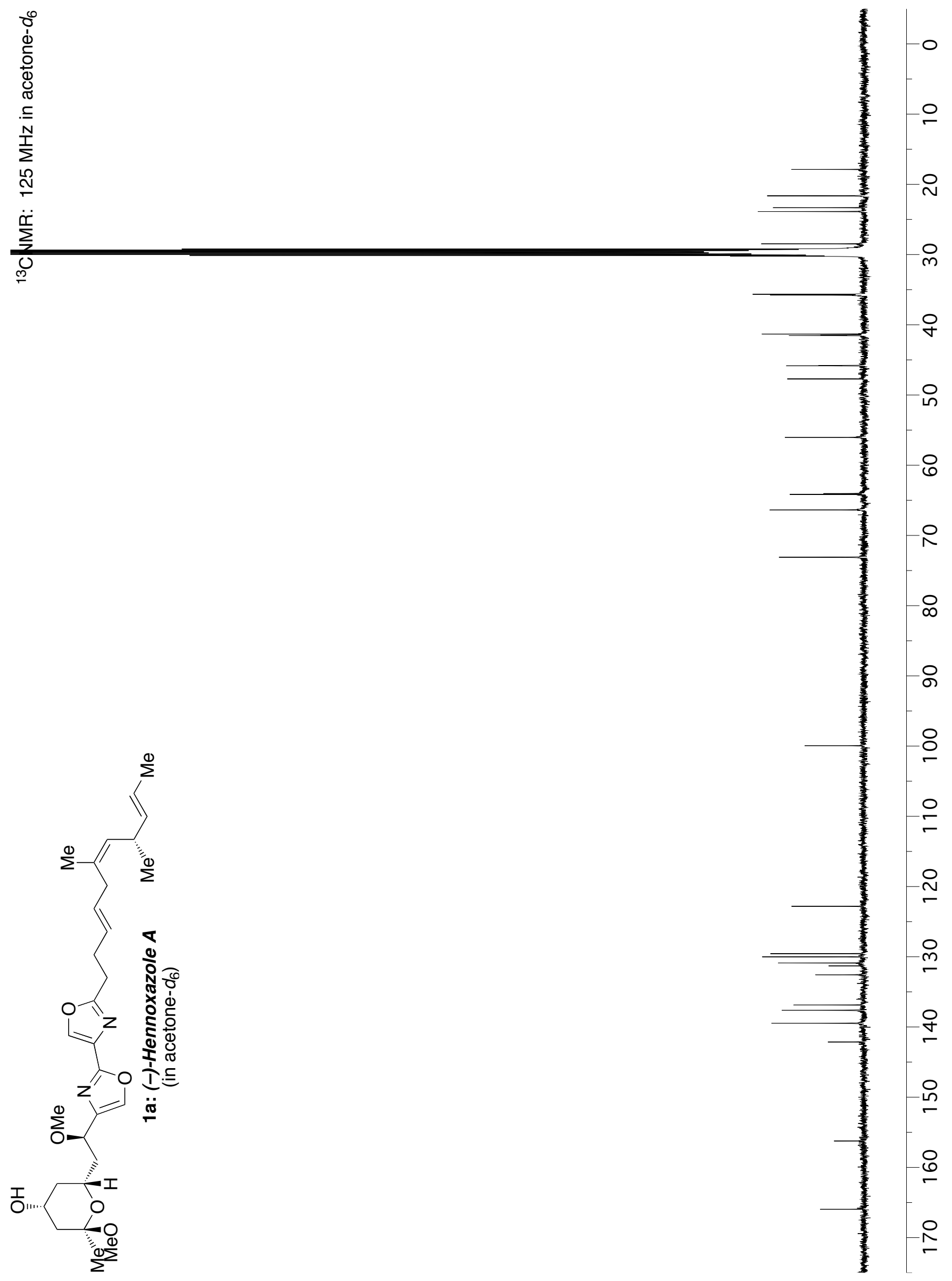




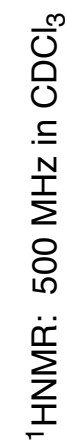

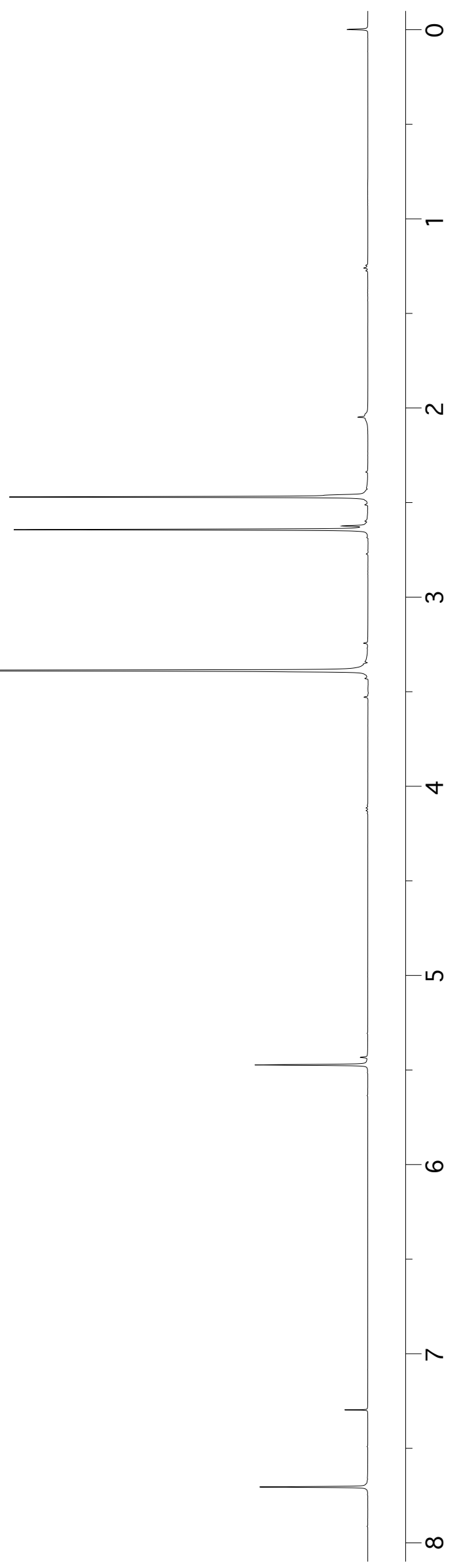




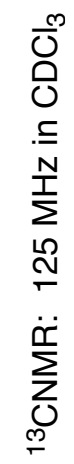
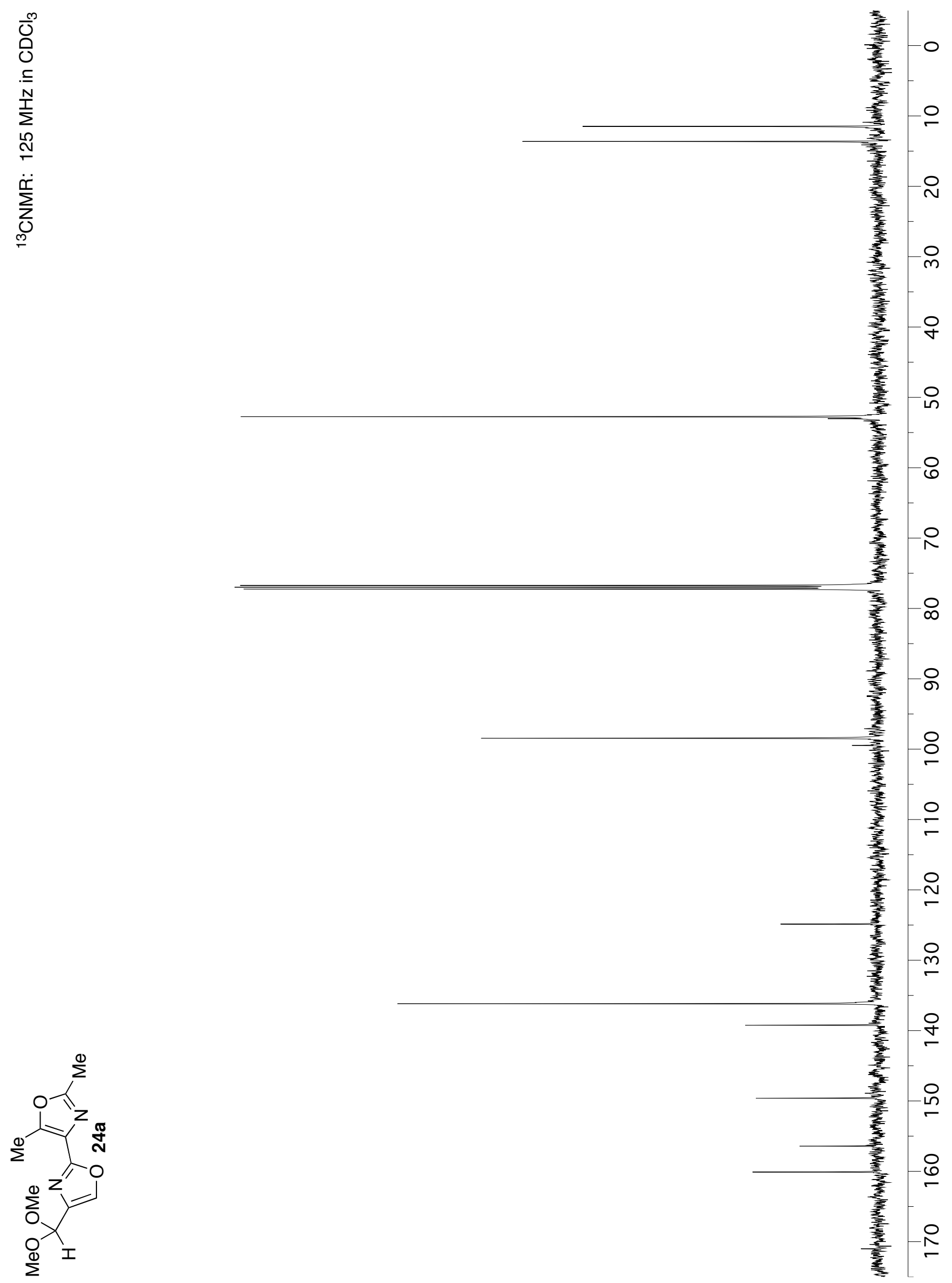


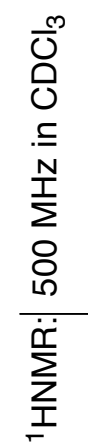

$\rightarrow+$

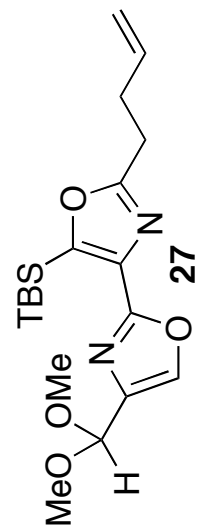




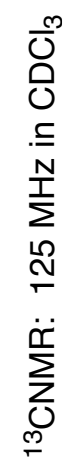
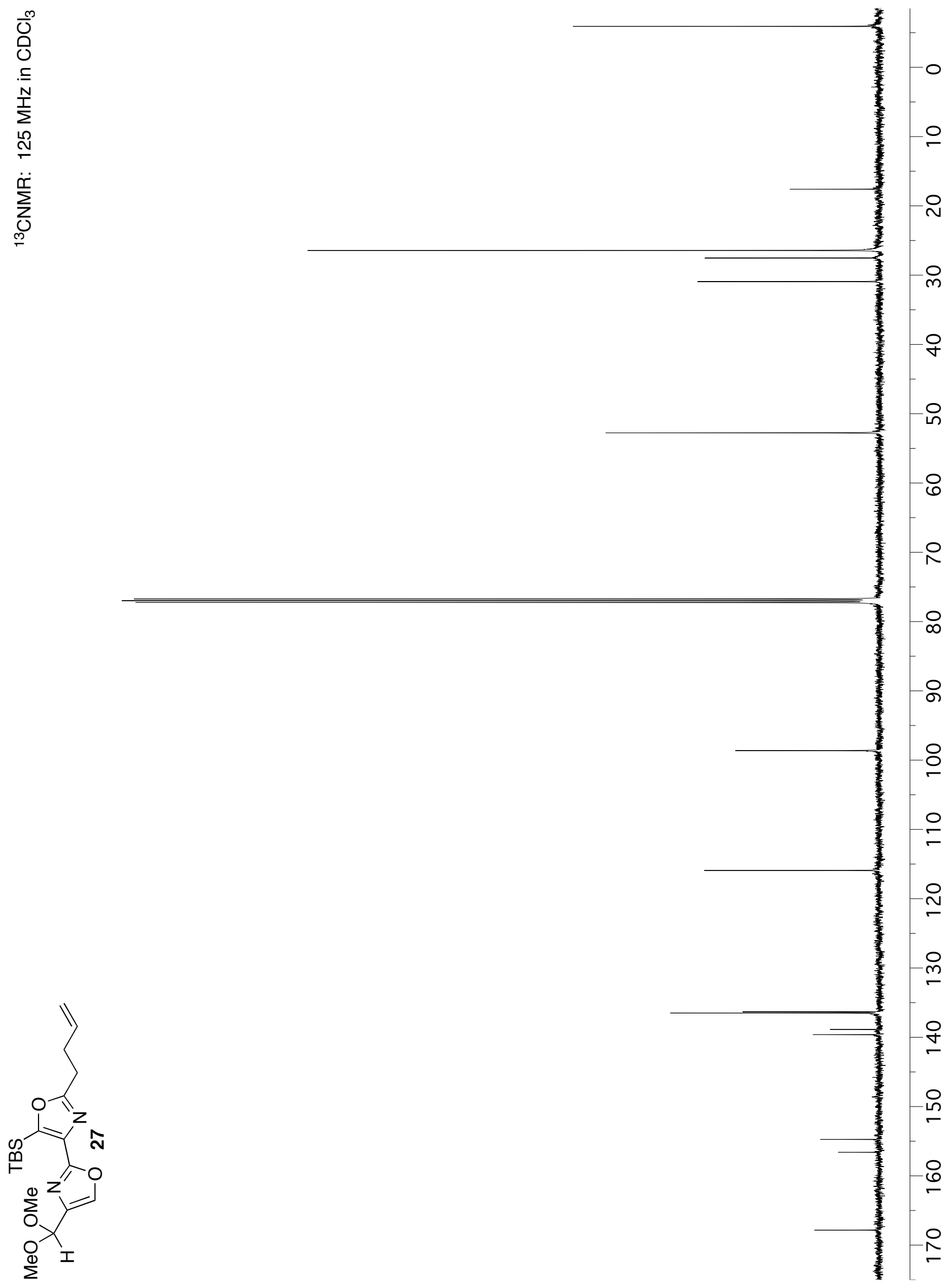


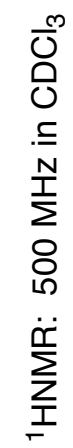

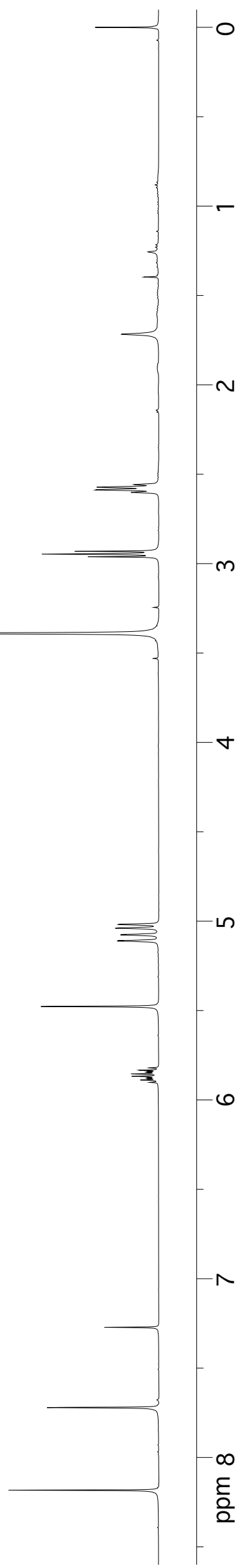




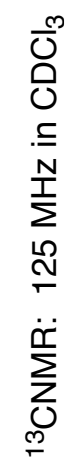

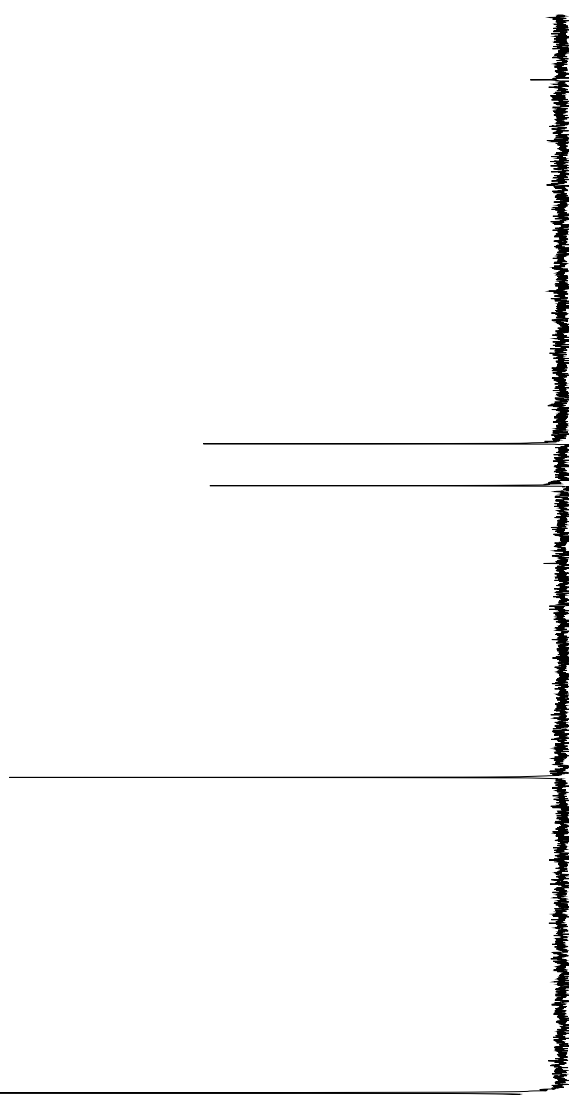

+4

$-0$

으

음

- 을

$-\stackrel{8}{8}$

ㅇำ

8

$\stackrel{0}{1}$
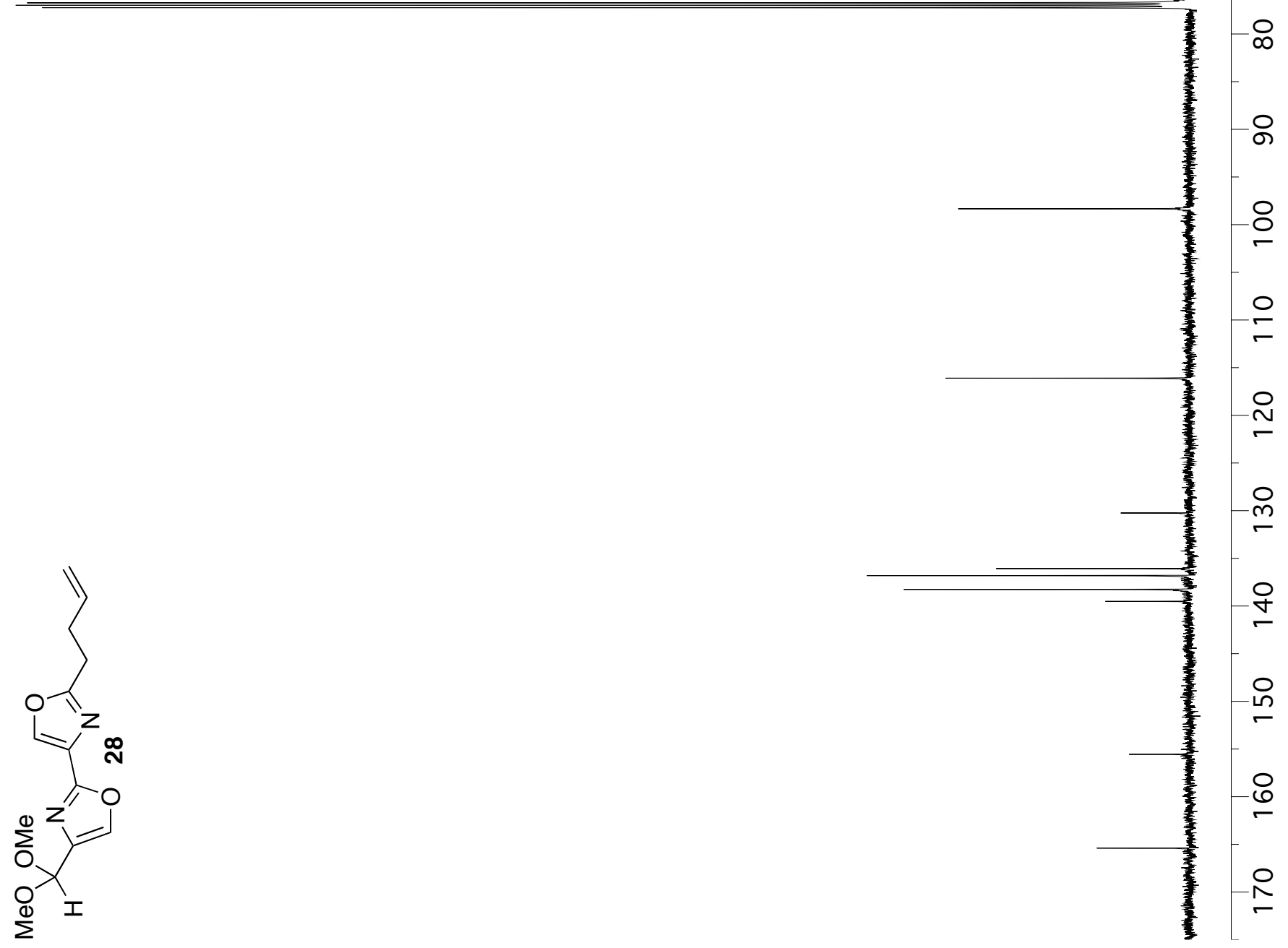


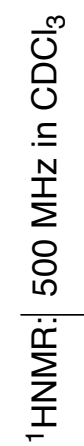
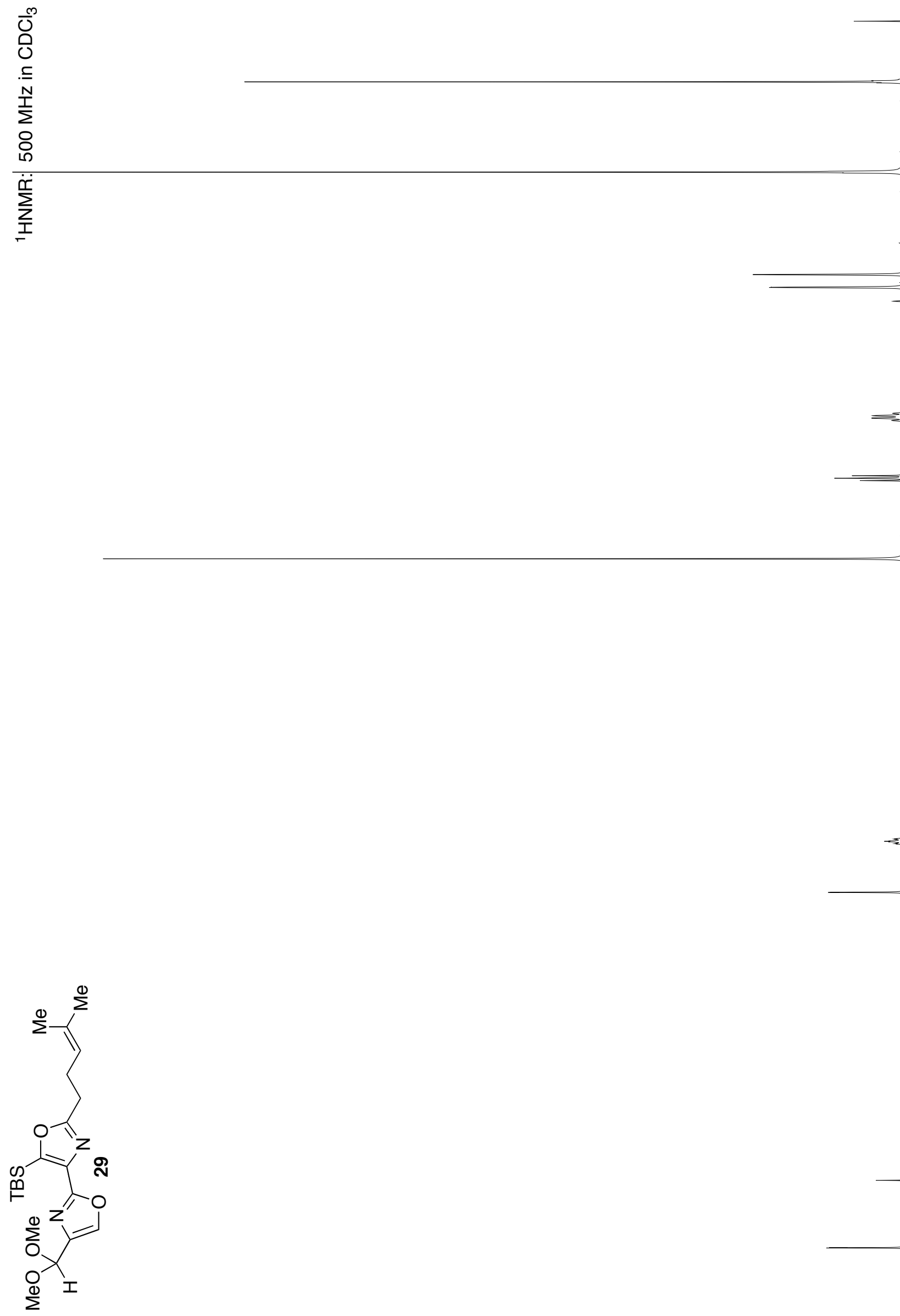


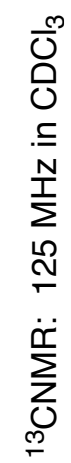

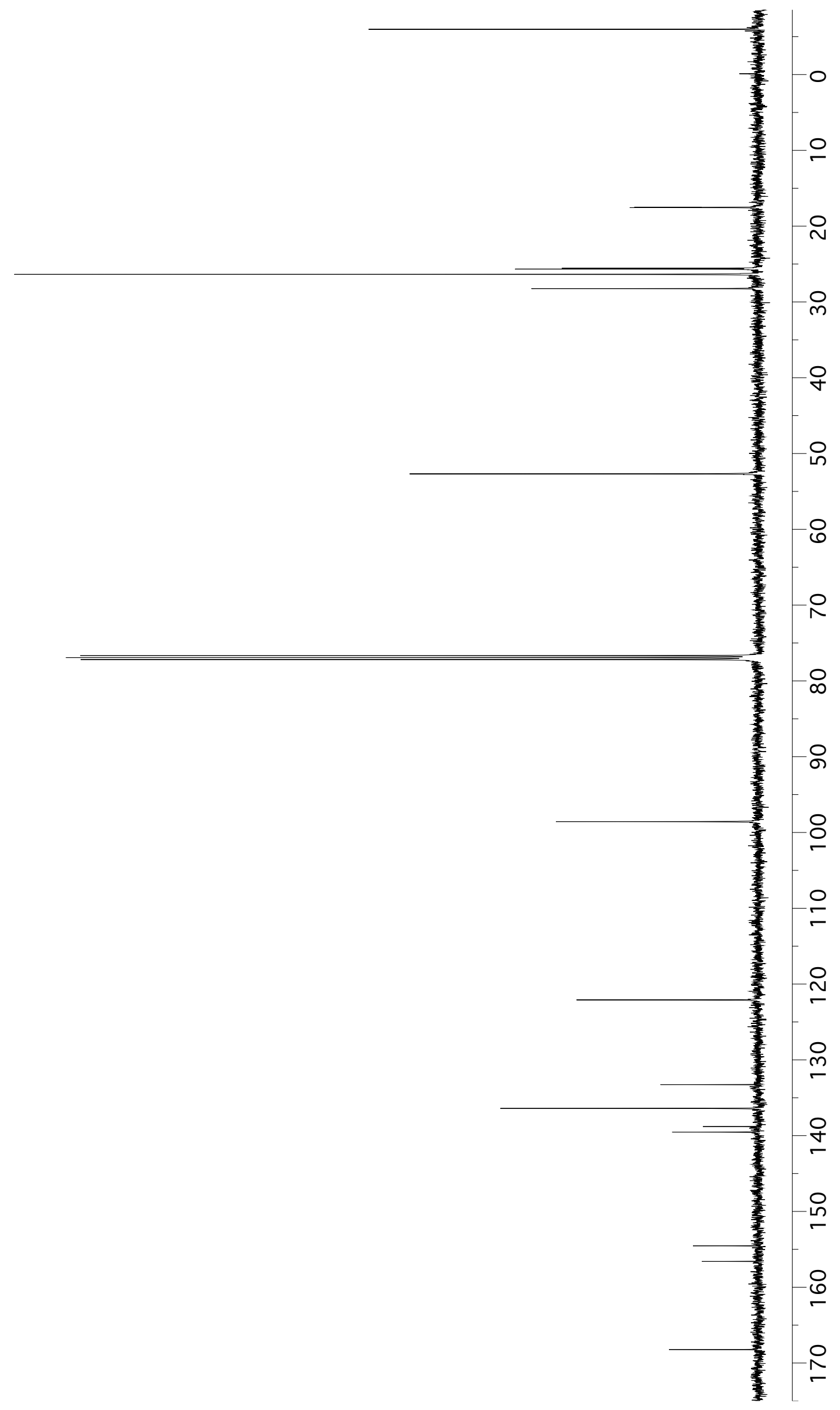




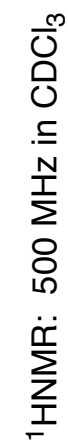

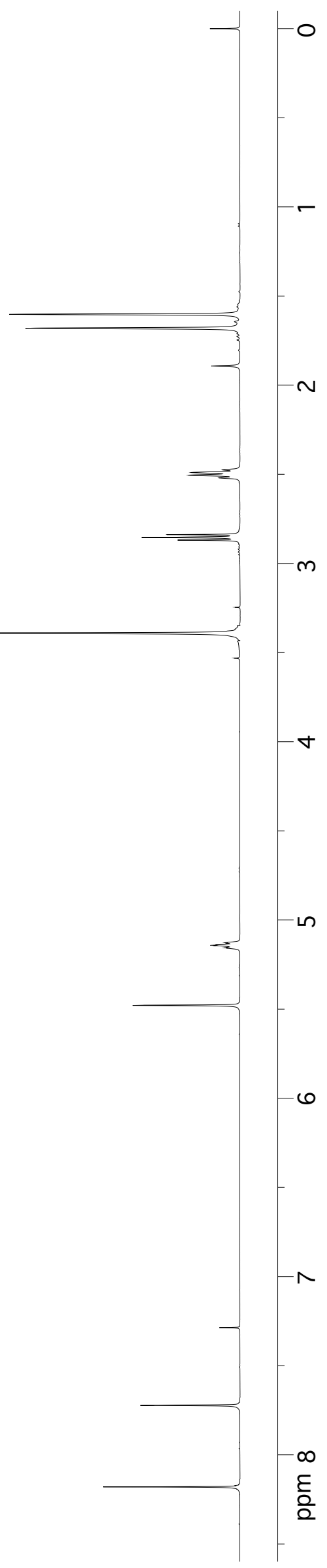




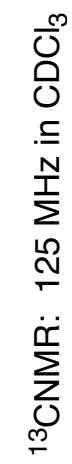

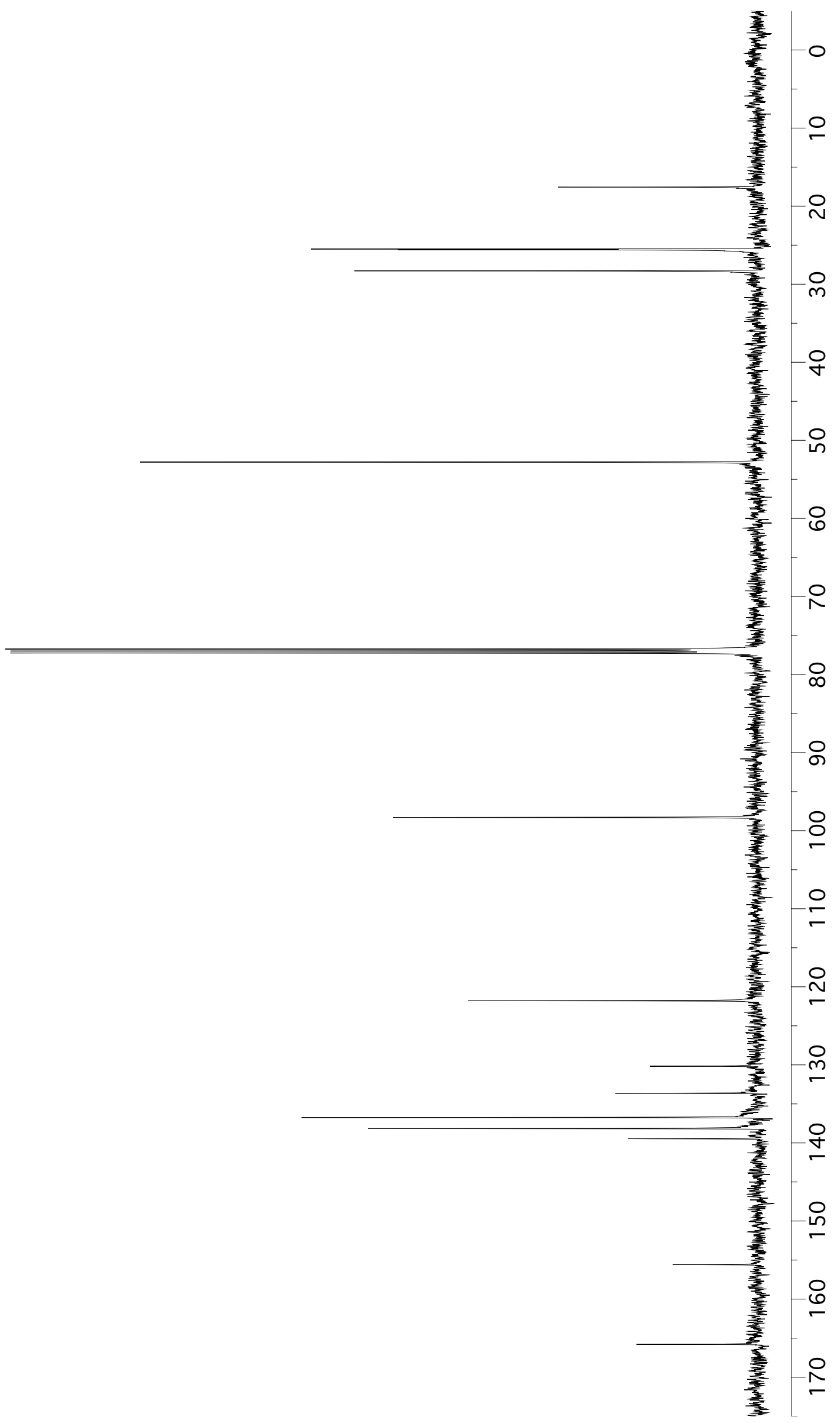




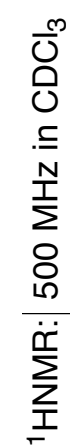

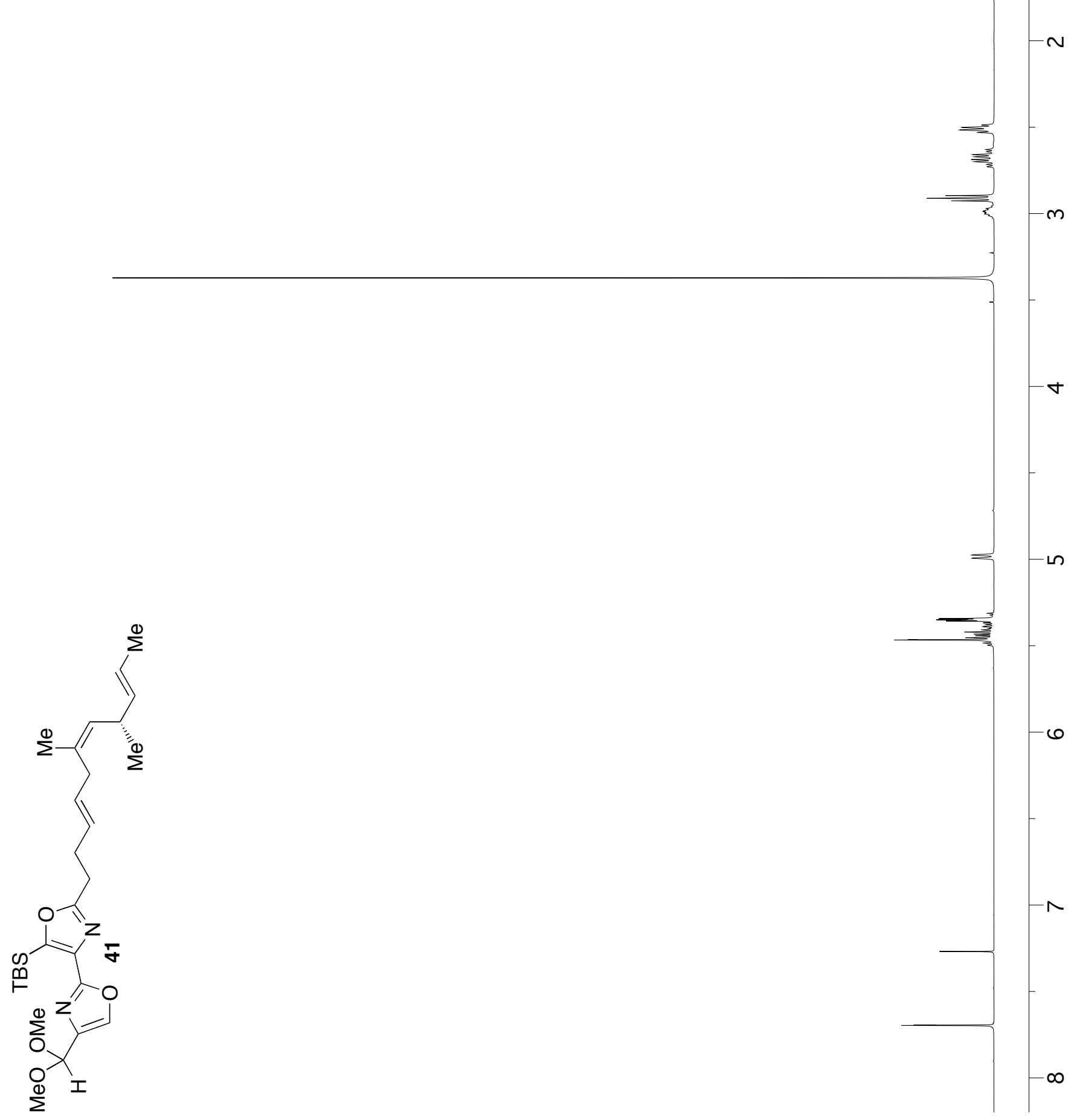

- 


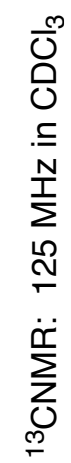

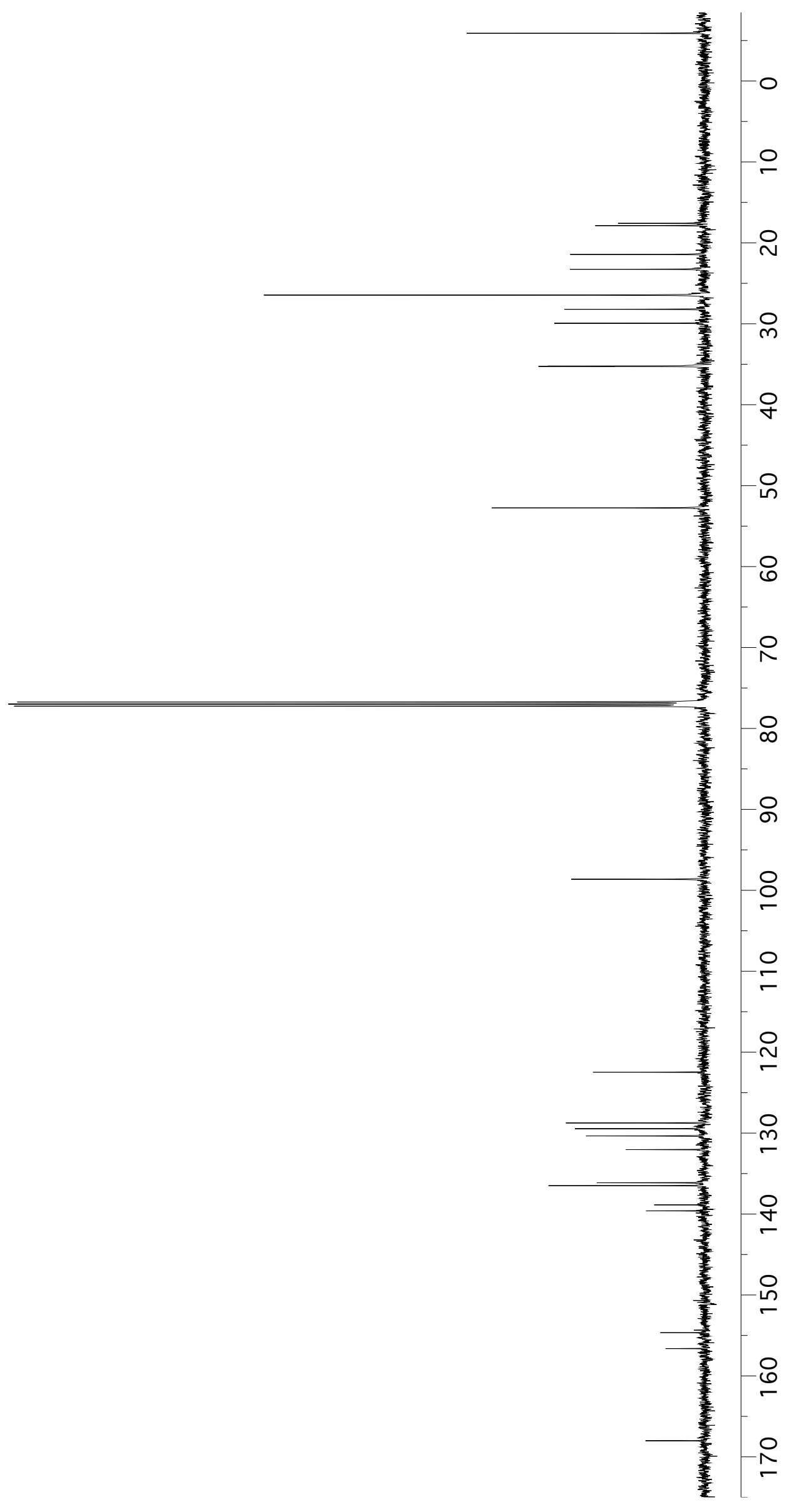




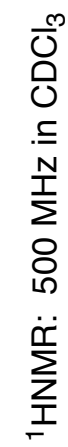

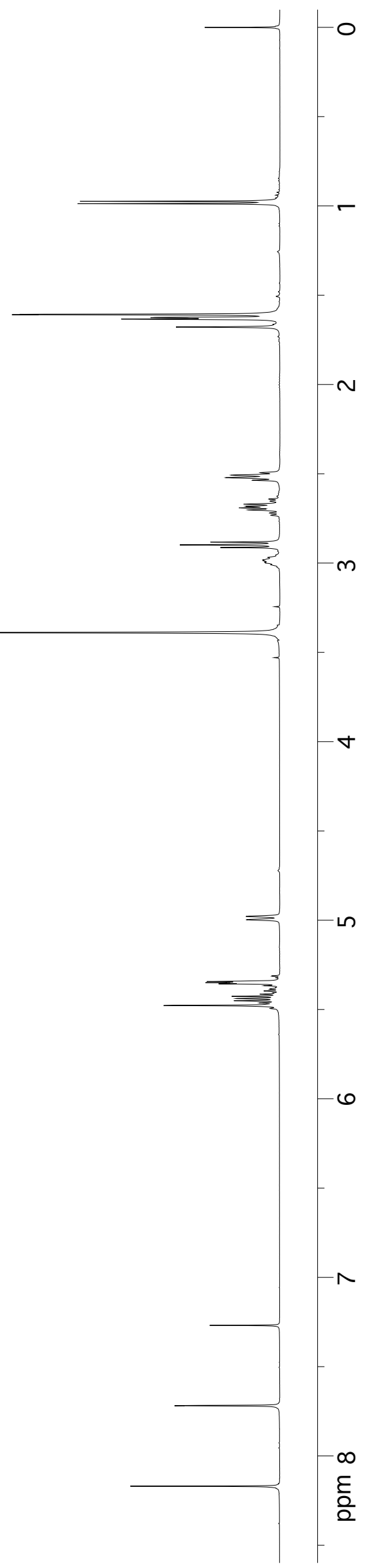




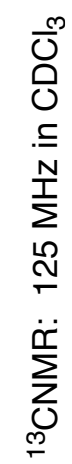
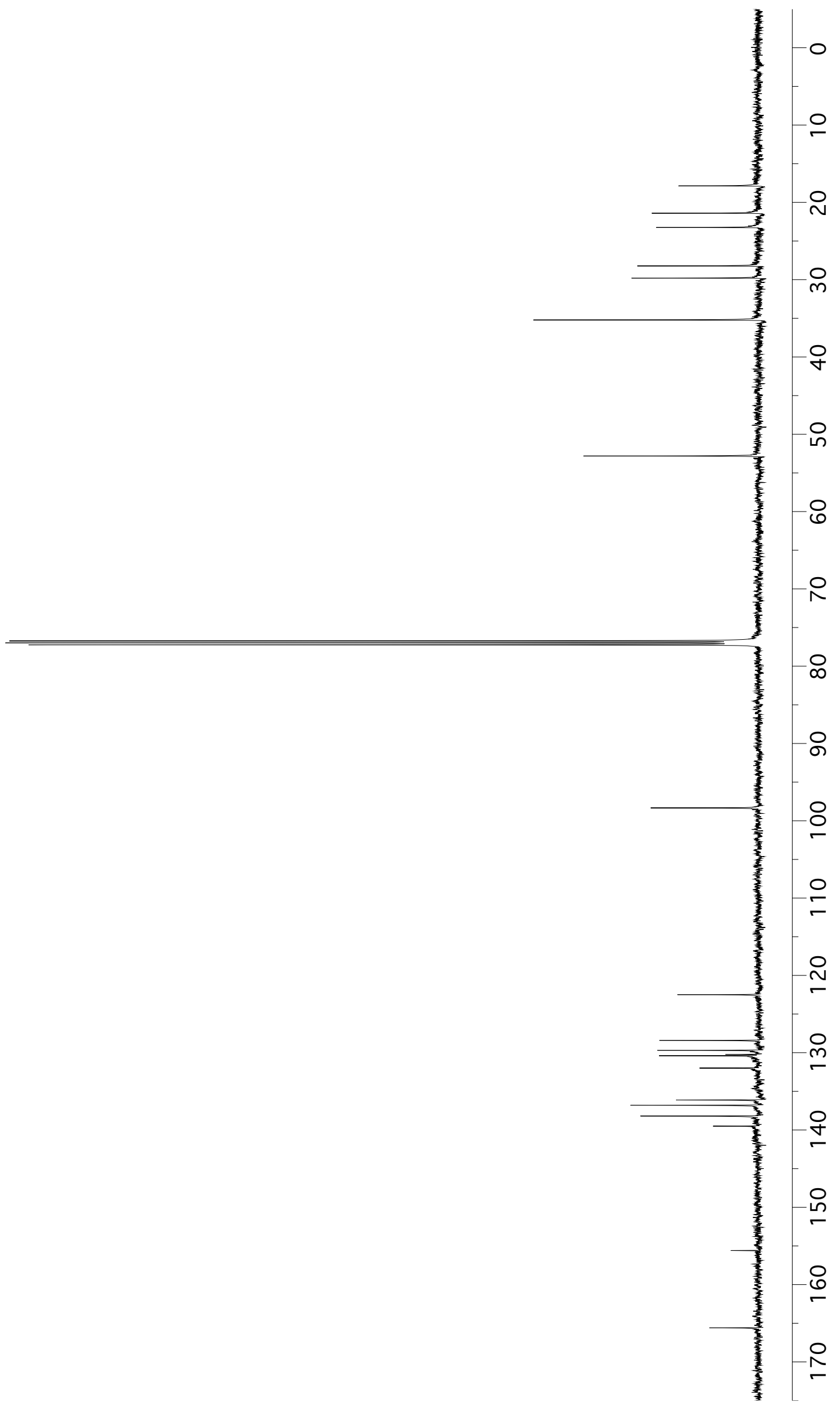

$-0$ 


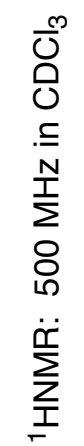

$\rightarrow+$
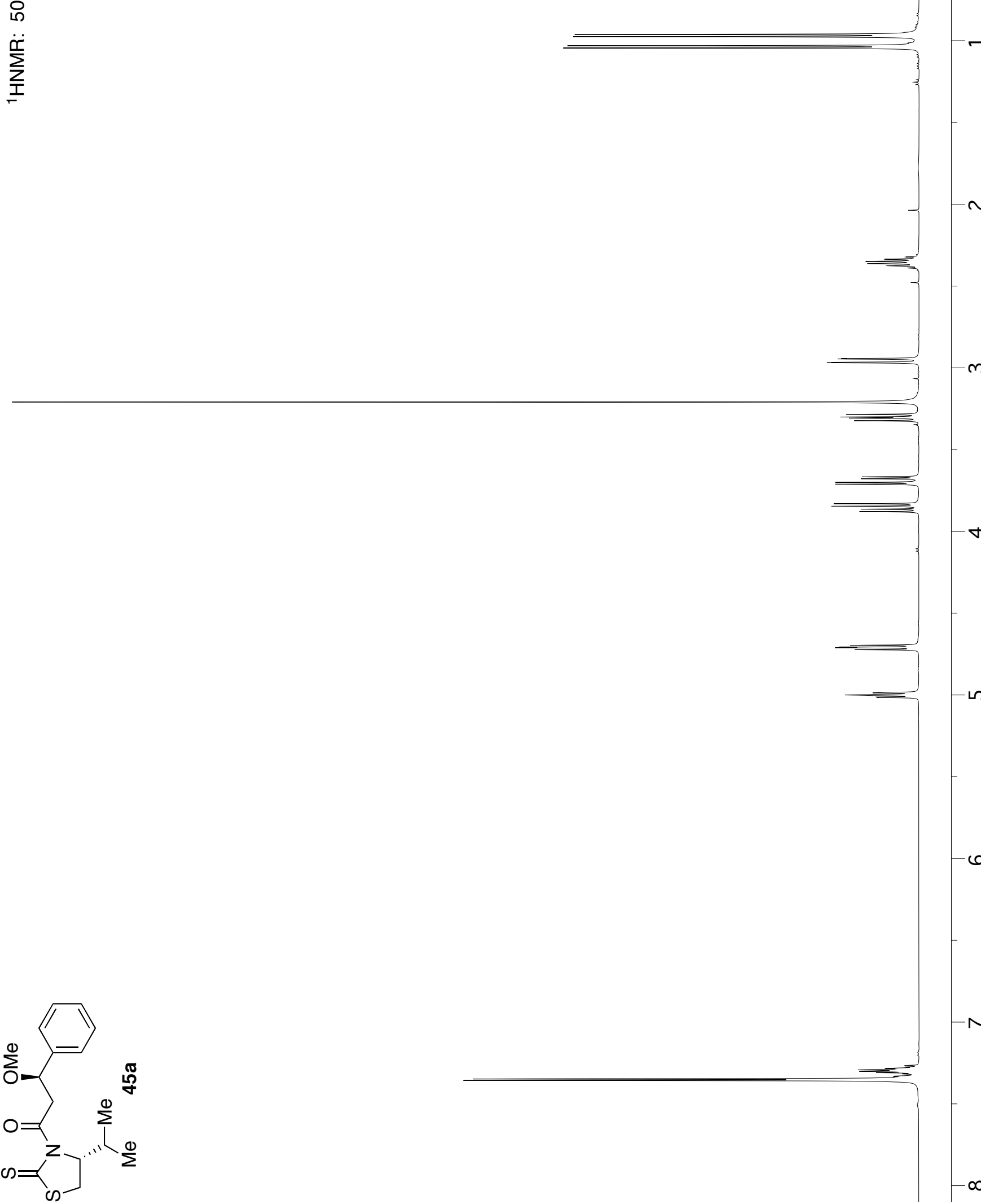

寸

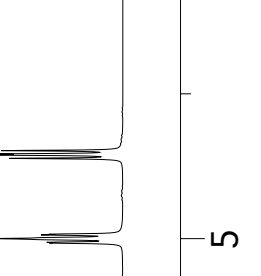




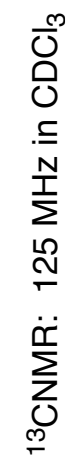
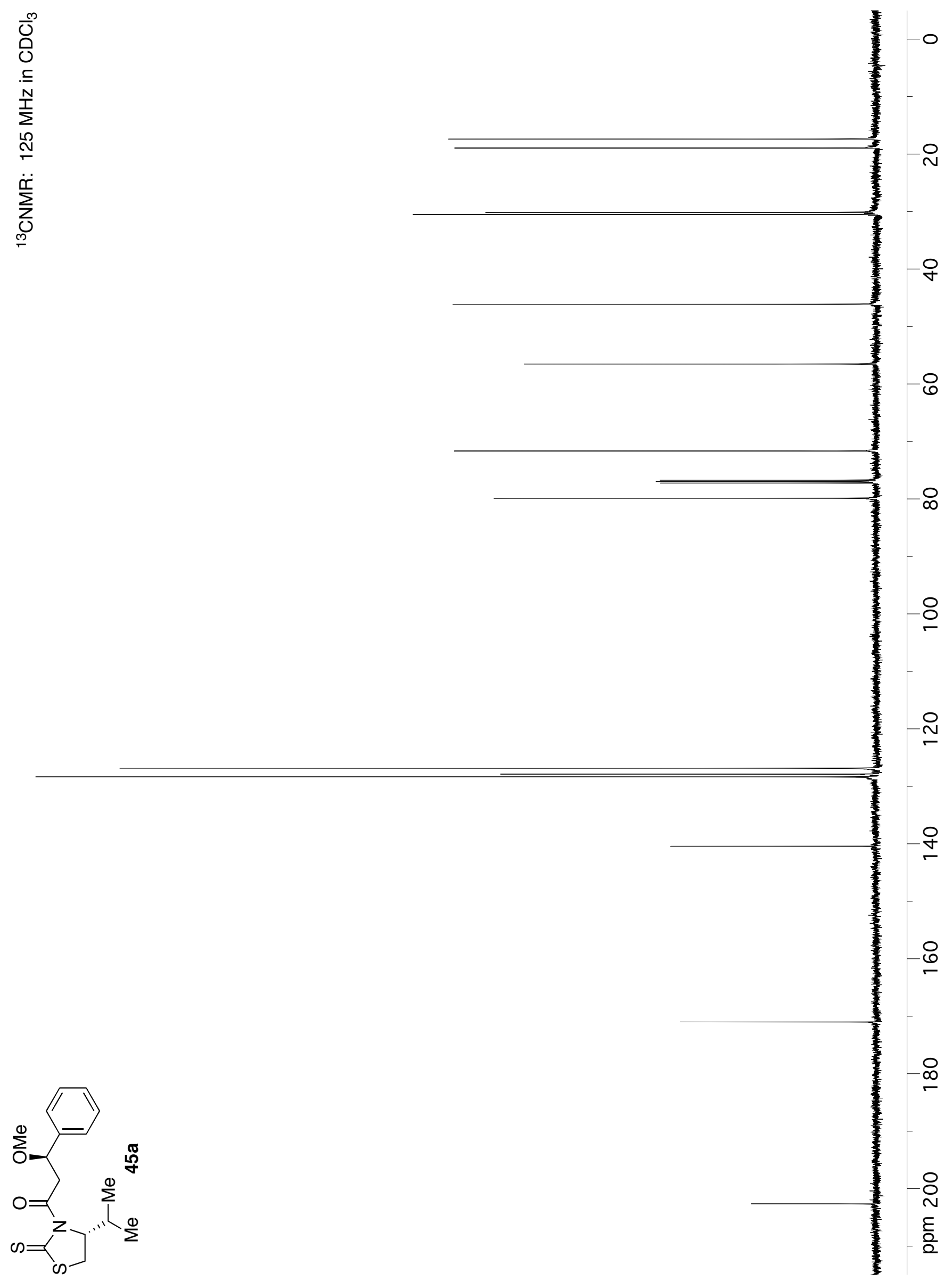


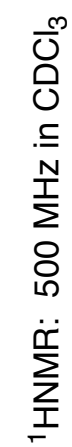

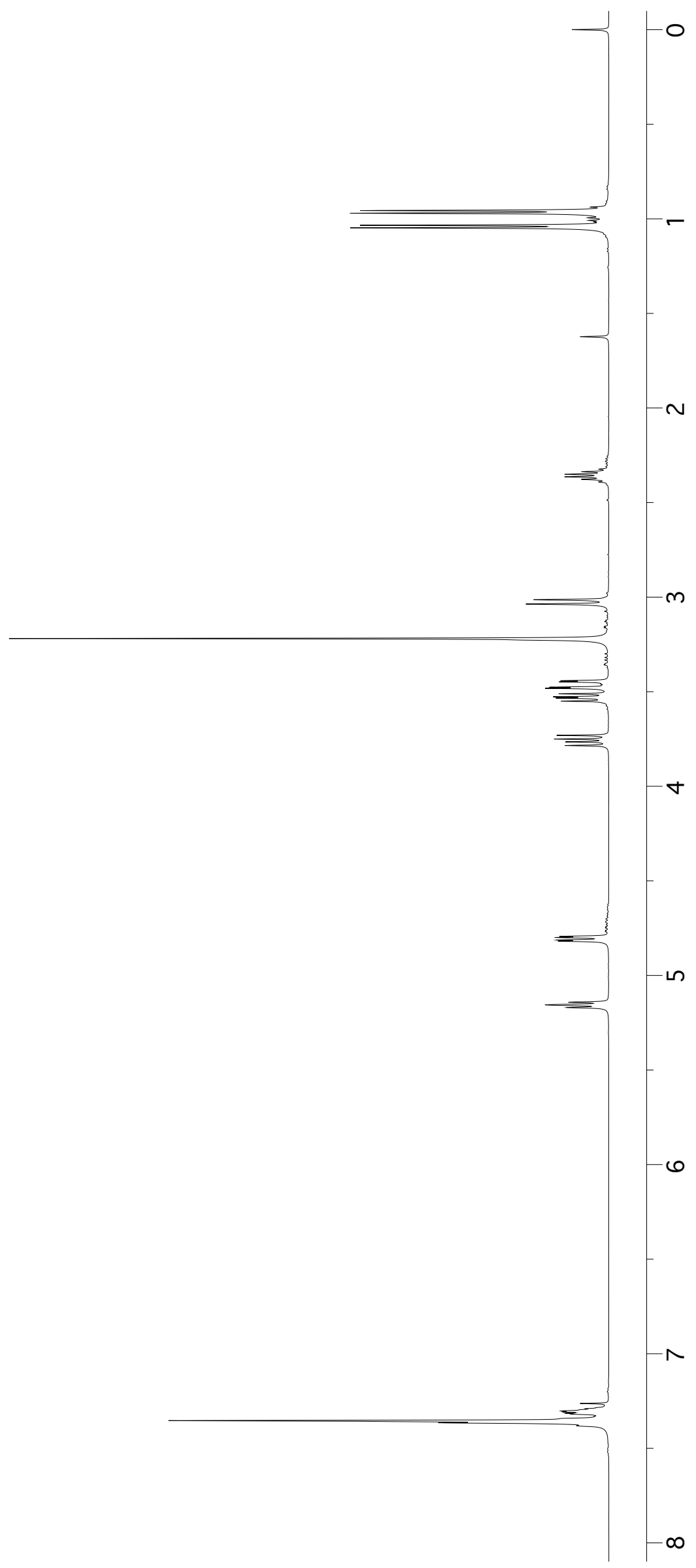




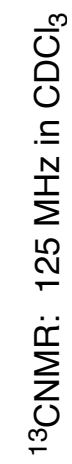

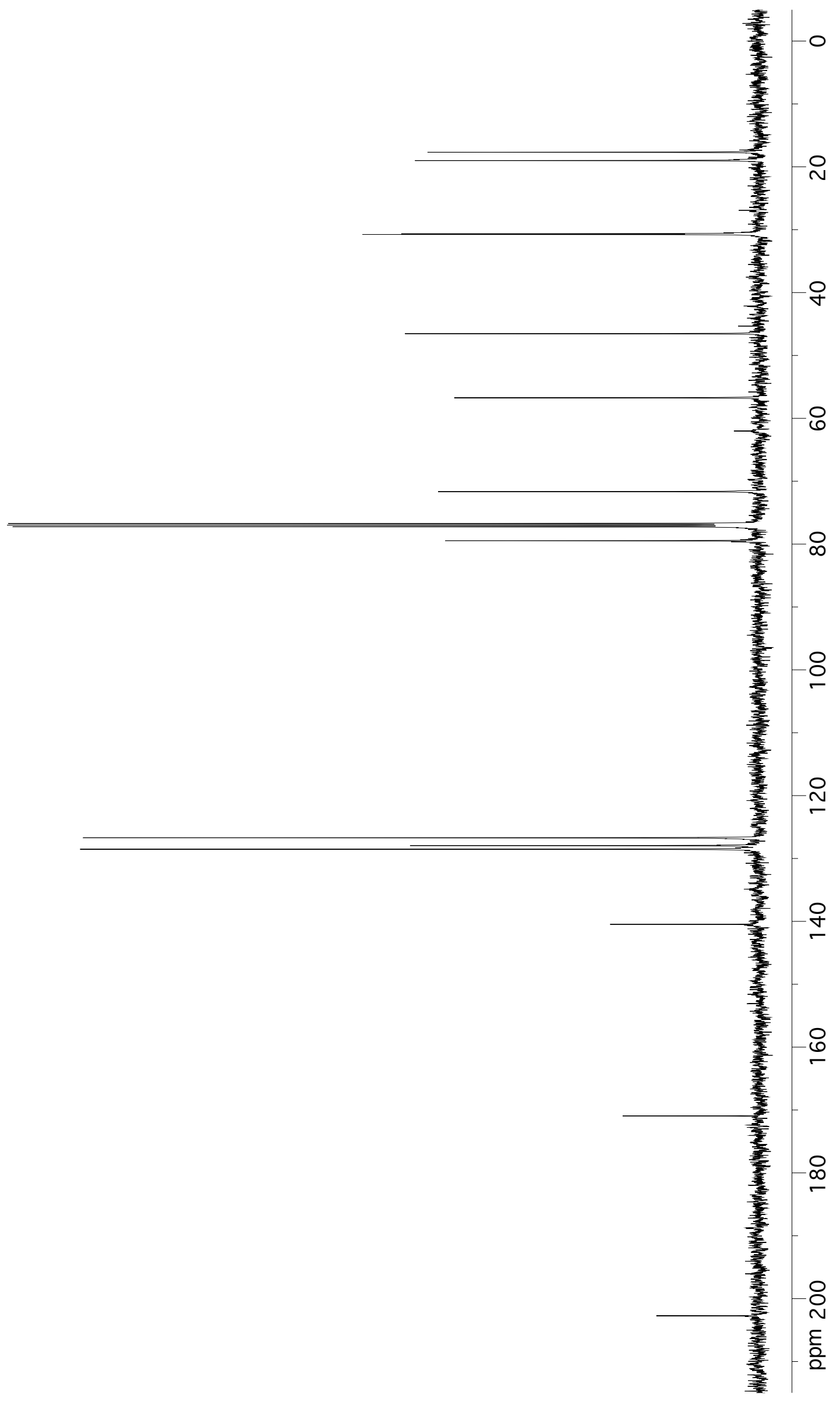




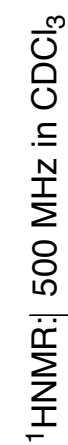
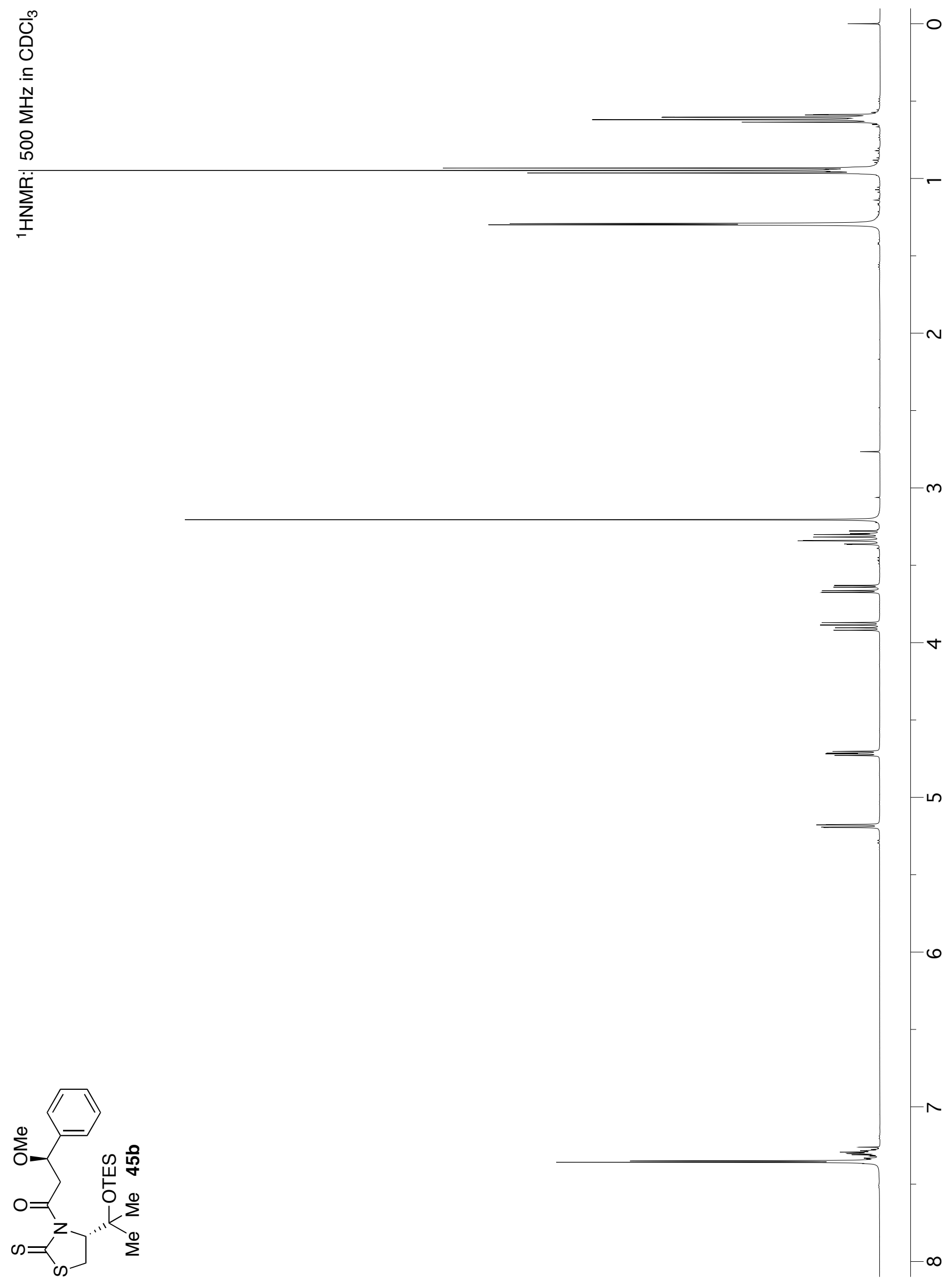


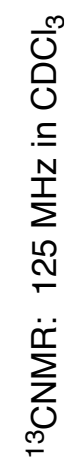

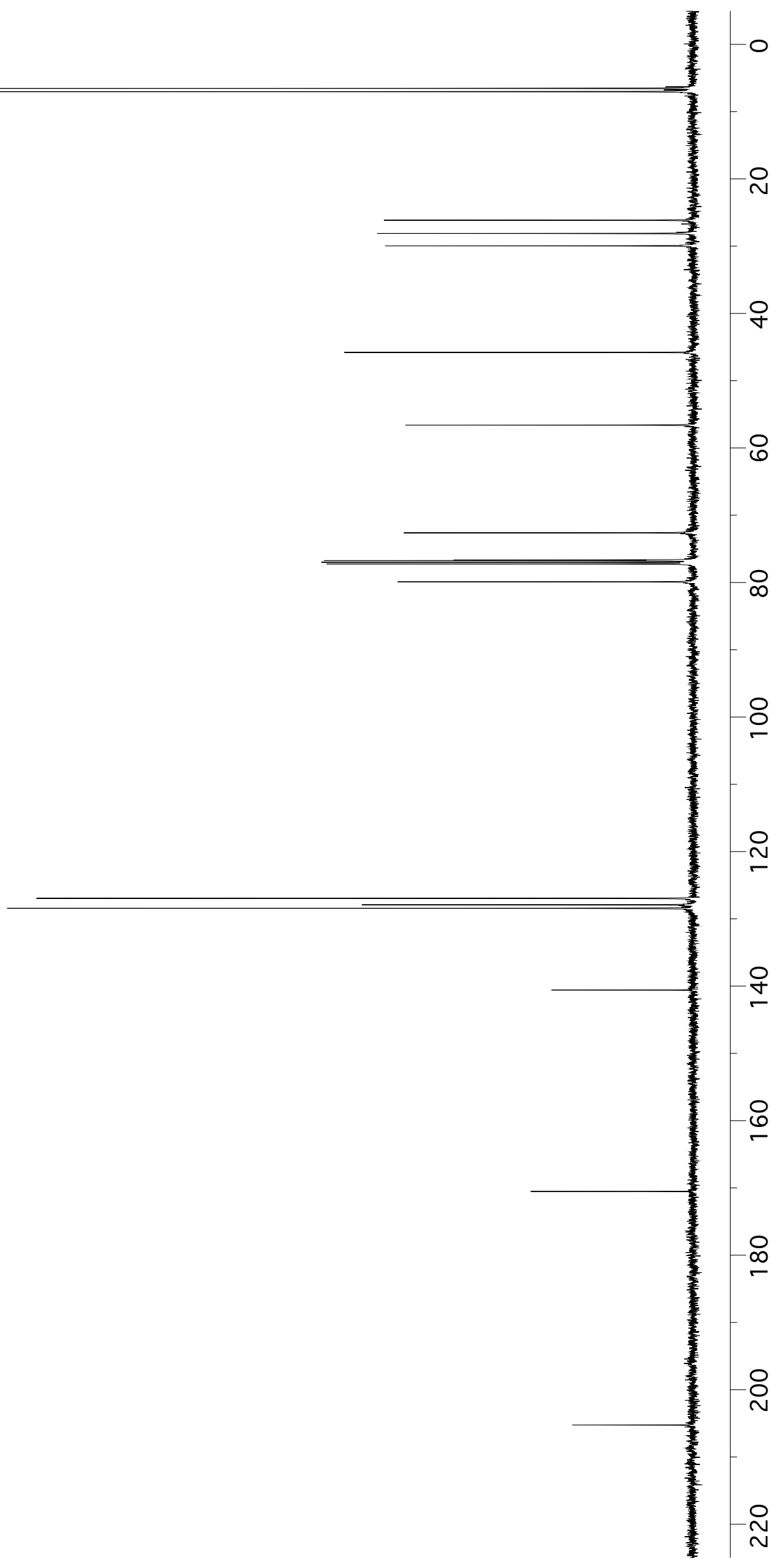




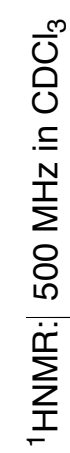

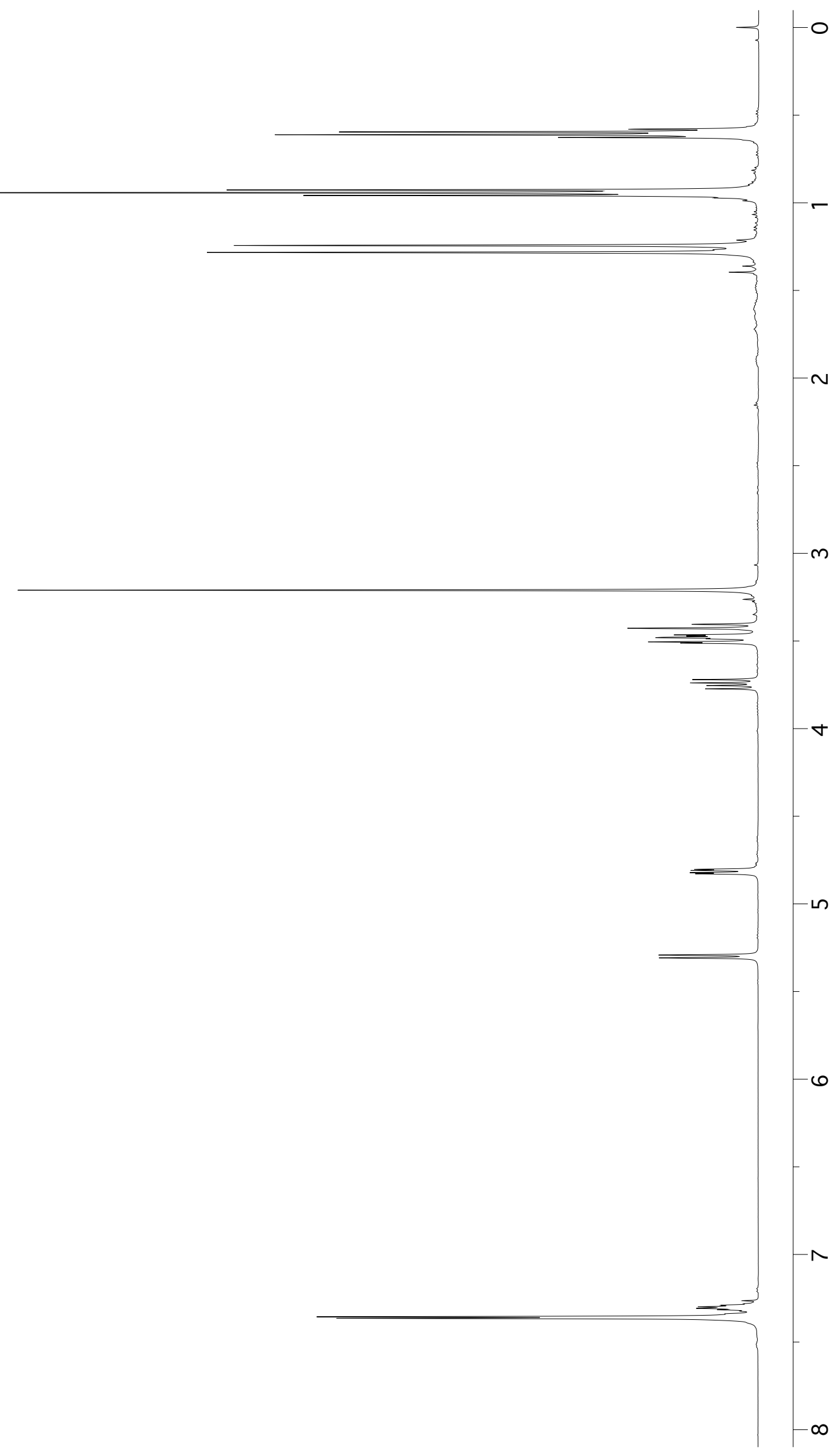




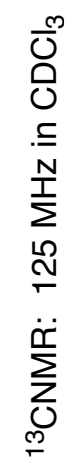

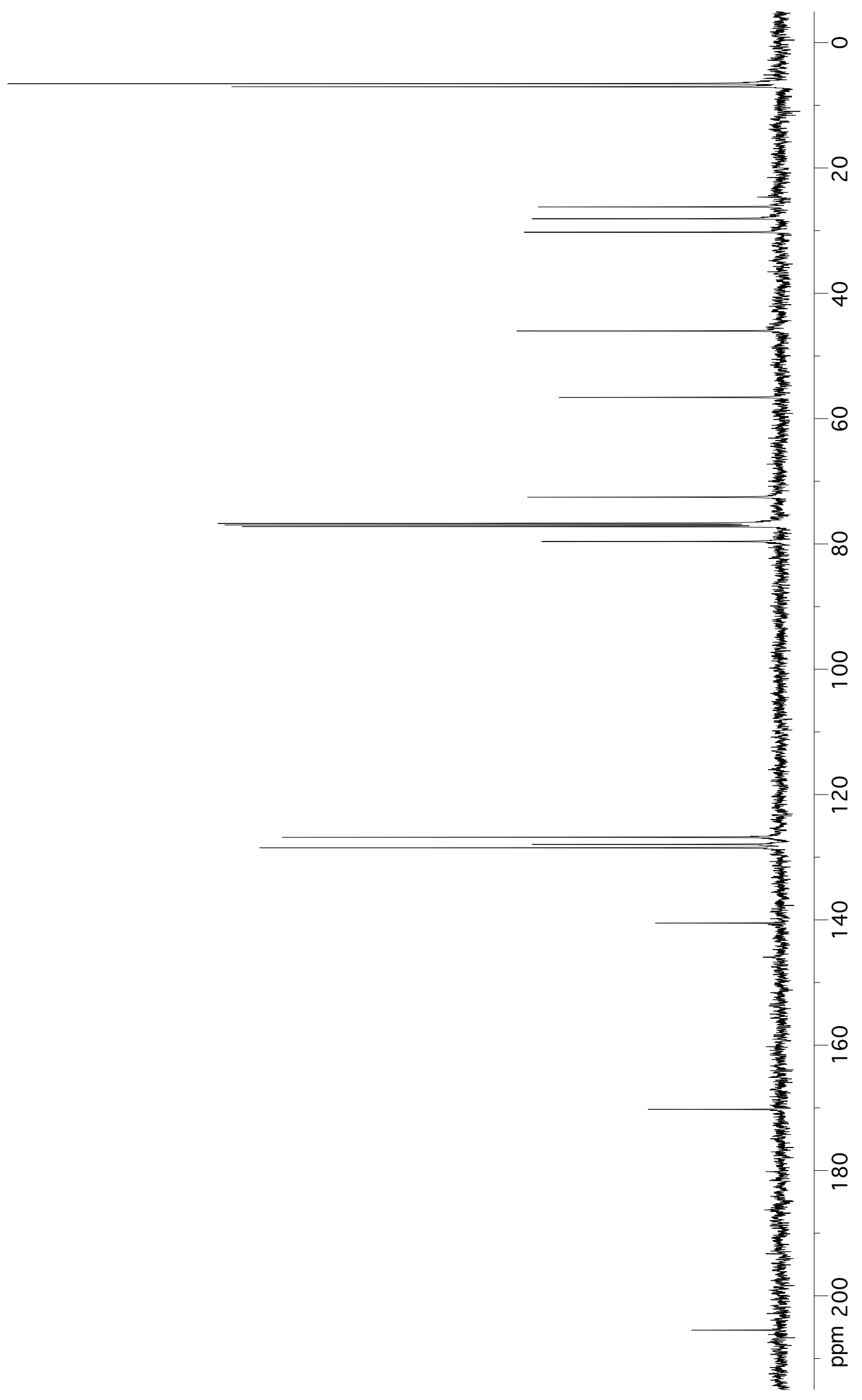




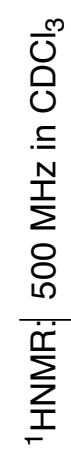

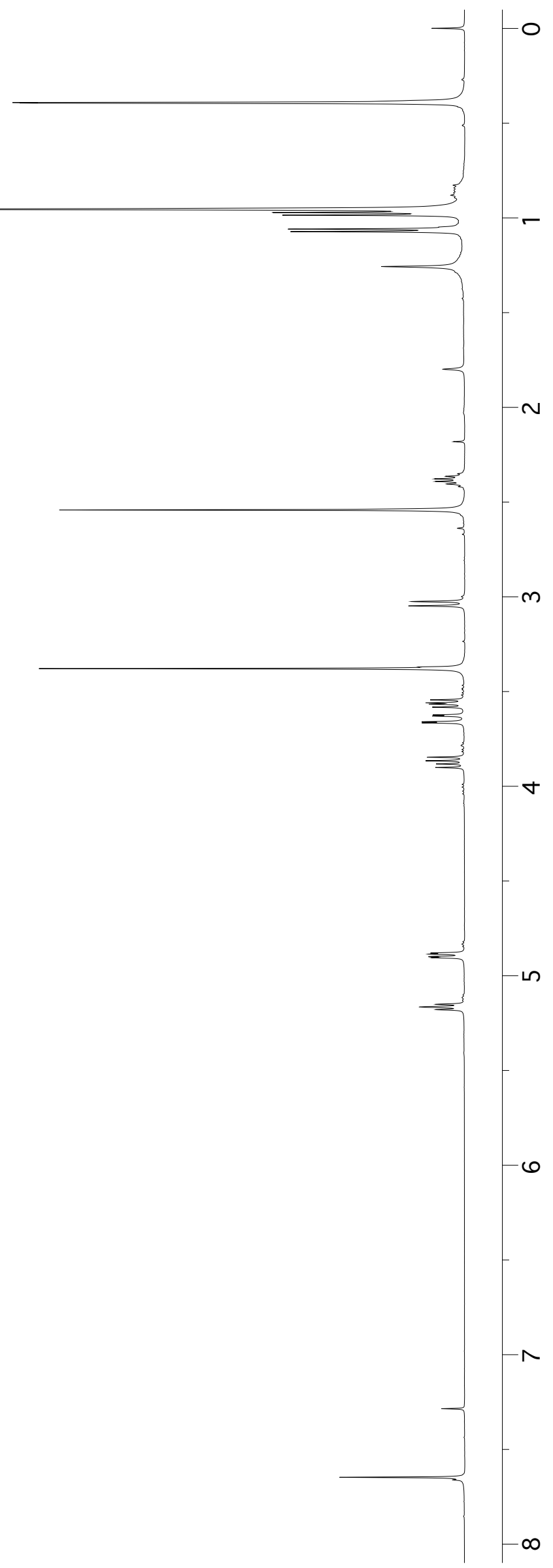




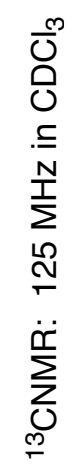

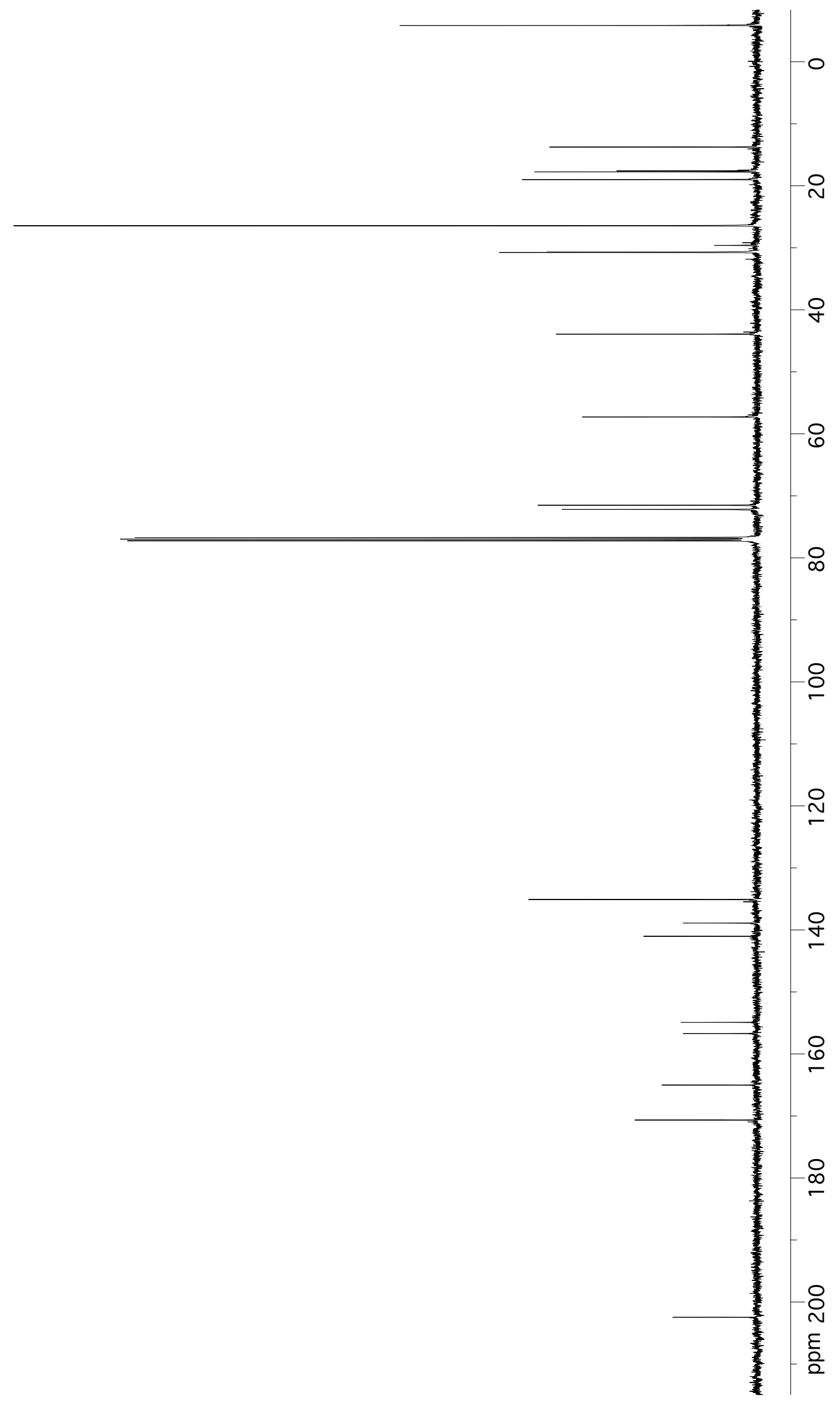




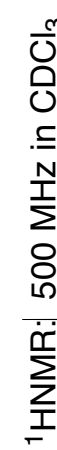
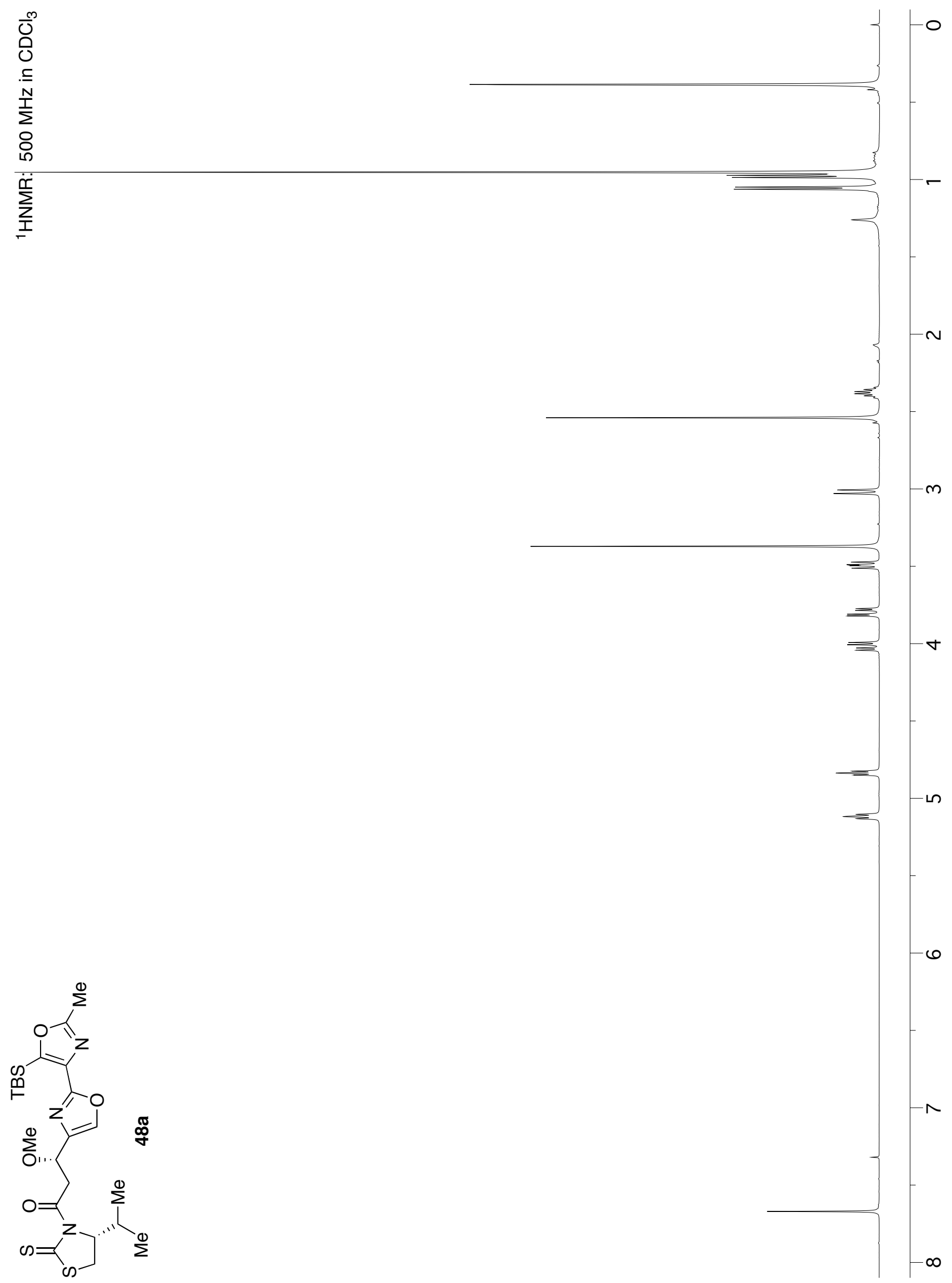


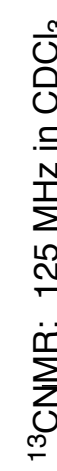

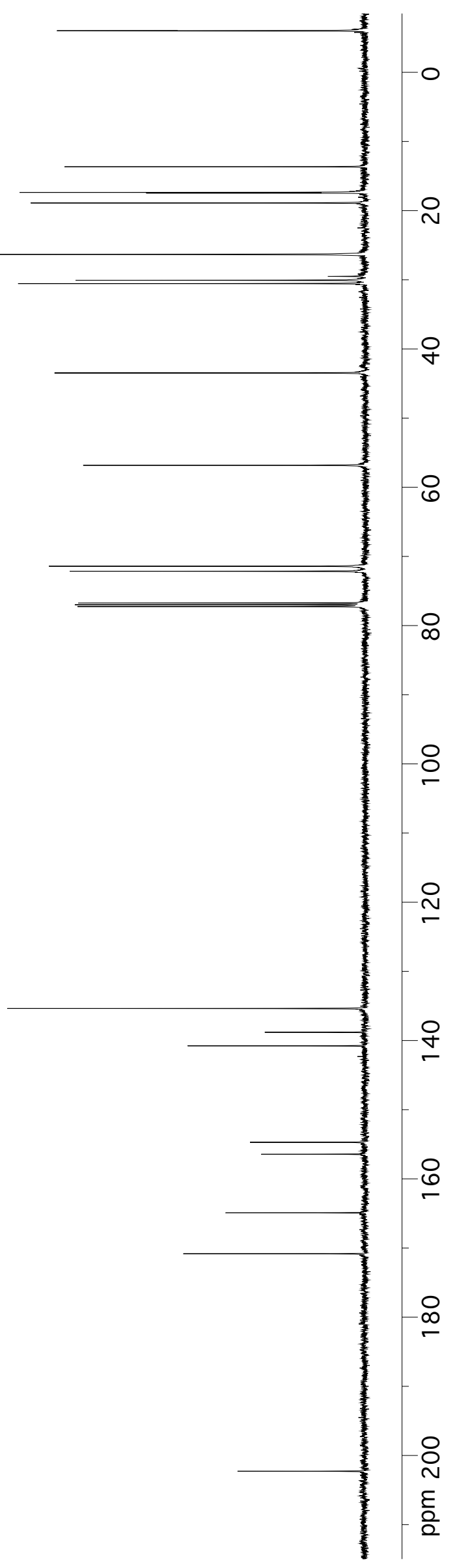

